\title{
Physical principles for scalable neural recording
}

\author{
Adam H. Marblestone ${ }^{1,2 * t}$, Bradley M. Zamft ${ }^{3 \dagger}$, Yael G. Maguire ${ }^{3,4}$, Mikhail G. Shapiro $^{5}$, \\ Thaddeus R. Cybulski ${ }^{6}$, Joshua I. Glaser ${ }^{6}$, Dario Amodei ${ }^{7}$, P Benjamin Stranges $^{3}$, Reza Kalhor ${ }^{3}$, \\ David A. Dalrymple ${ }^{1,8,9}$, Dongjin Seo ${ }^{10}$, Elad Alon ${ }^{10}$, Michel M. Maharbiz ${ }^{10}$, Jose M. Carmena ${ }^{10,11}$, \\ Jan M. Rabaey ${ }^{10}$, Edward S. Boyden ${ }^{9,12 \neq}$, George M. Church ${ }^{1,2,3 \neq}$ and Konrad P. Kording ${ }^{13,14 \neq}$ \\ 'Biophysics Program, Harvard University, Boston, MA, USA \\ ${ }^{2}$ Wyss Institute for Biologically Inspired Engineering at Harvard University, Boston, MA, USA \\ ${ }^{3}$ Department of Genetics, Harvard Medical School, Boston, MA, USA \\ ${ }^{4}$ Plum Labs LLC, Cambridge, MA, USA \\ ${ }^{5}$ Division of Chemistry and Chemical Engineering, California Institute of Technology, Pasadena, CA, USA \\ ${ }^{6}$ Interdepartmental Neuroscience Program, Northwestern University, Chicago, IL, USA \\ 7 Department of Radiology, Stanford University, Palo Alto, CA, USA \\ ${ }^{8}$ Nemaload, San Francisco, CA, USA \\ ${ }^{9}$ Media Laboratory, Massachusetts Institute of Technology, Cambridge, MA, USA \\ ${ }^{10}$ Department of Electrical Engineering and Computer Sciences, University of California at Berkeley, Berkeley, CA, USA \\ $"$ Helen Wills Neuroscience Institute, University of California at Berkeley, Berkeley, CA, USA \\ 12 Departments of Brain and Cognitive Sciences and Biological Engineering, Massachusetts Institute of Technology, Cambridge, MA, USA \\ ${ }^{13}$ Departments of Physical Medicine and Rehabilitation and of Physiology, Northwestern University Feinberg School of Medicine, Chicago, IL, USA \\ ${ }^{14}$ Sensory Motor Performance Program, The Rehabilitation Institute of Chicago, Chicago, IL, USA
}

\section{Edited by:}

David Hansel, University of Paris,

France

Reviewed by:

Simon R. Schultz, Imperial College

London, UK

Venkatesh N. Murthy, Harvard

University, USA

*Correspondence:

Adam H. Marblestone, Biophysics Program, Harvard University and

Wyss Institute, CLSB 530/4D, 3

Blackfan Circle, Boston, MA 02115

USA

e-mail: adam.h.marblestone@

gmail.com

${ }^{\dagger}$ Joint first authors.

$\neq$ Joint last authors.
Simultaneously measuring the activities of all neurons in a mammalian brain at millisecond resolution is a challenge beyond the limits of existing techniques in neuroscience. Entirely new approaches may be required, motivating an analysis of the fundamental physical constraints on the problem. We outline the physical principles governing brain activity mapping using optical, electrical, magnetic resonance, and molecular modalities of neural recording. Focusing on the mouse brain, we analyze the scalability of each method, concentrating on the limitations imposed by spatiotemporal resolution, energy dissipation, and volume displacement. Based on this analysis, all existing approaches require orders of magnitude improvement in key parameters. Electrical recording is limited by the low multiplexing capacity of electrodes and their lack of intrinsic spatial resolution, optical methods are constrained by the scattering of visible light in brain tissue, magnetic resonance is hindered by the diffusion and relaxation timescales of water protons, and the implementation of molecular recording is complicated by the stochastic kinetics of enzymes. Understanding the physical limits of brain activity mapping may provide insight into opportunities for novel solutions. For example, unconventional methods for delivering electrodes may enable unprecedented numbers of recording sites, embedded optical devices could allow optical detectors to be placed within a few scattering lengths of the measured neurons, and new classes of molecularly engineered sensors might obviate cumbersome hardware architectures. We also study the physics of powering and communicating with microscale devices embedded in brain tissue and find that, while radio-frequency electromagnetic data transmission suffers from a severe power-bandwidth tradeoff, communication via infrared light or ultrasound may allow high data rates due to the possibility of spatial multiplexing. The use of embedded local recording and wireless data transmission would only be viable, however, given major improvements to the power efficiency of microelectronic devices.

Keywords: neural recording, brain activity mapping, electrical recording, optical methods, magnetic resonance imaging, molecular recording, embedded electronics

\begin{abstract}
"To understand in depth what is going on in a brain, we need tools that can fit inside or between neurons and transmit reports of neural events to receivers outside. We need observing instruments that are local, non-destructive and non-invasive, with rapid response, high band-width and high spatial resolution... There is no law of physics that declares such an observational tool to be impossible."
\end{abstract}

Freeman Dyson, Imagined Worlds, 1997

\section{INTRODUCTION}

Neuroscience depends on monitoring the electrical activities of neurons within functioning brains (Alivisatos et al., 2012; Bansal et al., 2012; Gerhard et al., 2013) and has advanced through steady improvements in the underlying observational tools. The number of neurons simultaneously recorded using wired electrodes, for example, has doubled every 7 years since the 1950s, 
currently allowing electrical observation of hundreds of neurons at sub-millisecond timescales (Stevenson and Kording, 2011). Recording techniques have also diversified: activity-dependent optical signals from neurons endowed with fluorescent indicators can be measured by photodetectors, and radio-frequency emissions from excited nuclear spins allow the construction of magnetic resonance images modulated by activity-dependent contrast mechanisms. Ideas for alternative methods have been proposed, including the direct recording of neural activities into information-bearing biopolymers (Kording, 2011; Zamft et al., 2012; Glaser et al., 2013).

Each modality of neural recording has characteristic advantages and disadvantages. Multi-electrode arrays enable the recording of $\sim 250$ neurons at sub-millisecond temporal resolutions. Optical microscopy can currently record $\sim 100,000$ neurons at a $1.25 \mathrm{~s}$ timescale in behaving larval zebrafish using light-sheet illumination (Ahrens et al., 2013), or hundreds to thousands of neurons at a $\sim 100 \mathrm{~ms}$ timescale in behaving mice using a 1-photon fiber scope (Ziv et al., 2013). Magnetic resonance imaging (MRI) allows non-invasive whole brain recordings at a $1 \mathrm{~s}$ timescale, but is far from single neuron spatial resolution, in part due to the use of hemodynamic contrast. Finally, molecular recording devices have been proposed for scalable physiological signal recording but have not yet been demonstrated in neurons (Kording, 2011; Zamft et al., 2012; Glaser et al., 2013).

Figure 1 illustrates the recording modalities studied here. While further development of these methods promises to be a crucial driver for future neuroscience research (Kandel et al., 2013), their fundamental scaling limits are not immediately obvious. Furthermore, inventing new technologies for scalable neural recording requires a quantitative understanding of the engineering problems that such technologies must solve, a landscape of constraints which should inform design decisions.

Our analysis is predicated on assumptions that enable us to estimate scaling limits. These include assumptions about basic properties of the brain, which are treated in Section 2, as well as those pertaining to the required measurement resolution and the limits to which a neural recording method may perturb brain tissue, which are treated in Section 3. Together, these considerations form the basis for our estimates of the prospects for scaling of neural recording technologies. We analyze four modalities of brain activity mapping-electrical, optical, magnetic resonance and molecular - in light of these assumptions, and conclude with a discussion on opportunities for new developments.

Importantly, our assumptions, analyses and the conclusions thereof are intended as first approximations and are subject to debate. We anticipate that as much can be learned from where our logic breaks down as from where it succeeds, and from methods to work around the limits imposed by our assumptions.

\section{BASIC CONSTRAINTS}

\subsection{MOUSE BRAIN}

The mouse brain contains $\sim 7.5 \times 10^{7}$ neurons in a volume of $\sim 420 \mathrm{~mm}^{3}$ (Vincent et al., 2010) and weighs about $0.5 \mathrm{~g}$. The packing density of neurons varies widely between brain regions. In the below, we will use a cell density of $\rho_{\text {neurons }} \approx 92,000 / \mathrm{mm}^{3}$, as measured for mouse cortex (Braitenberg and Schüz, 1991).

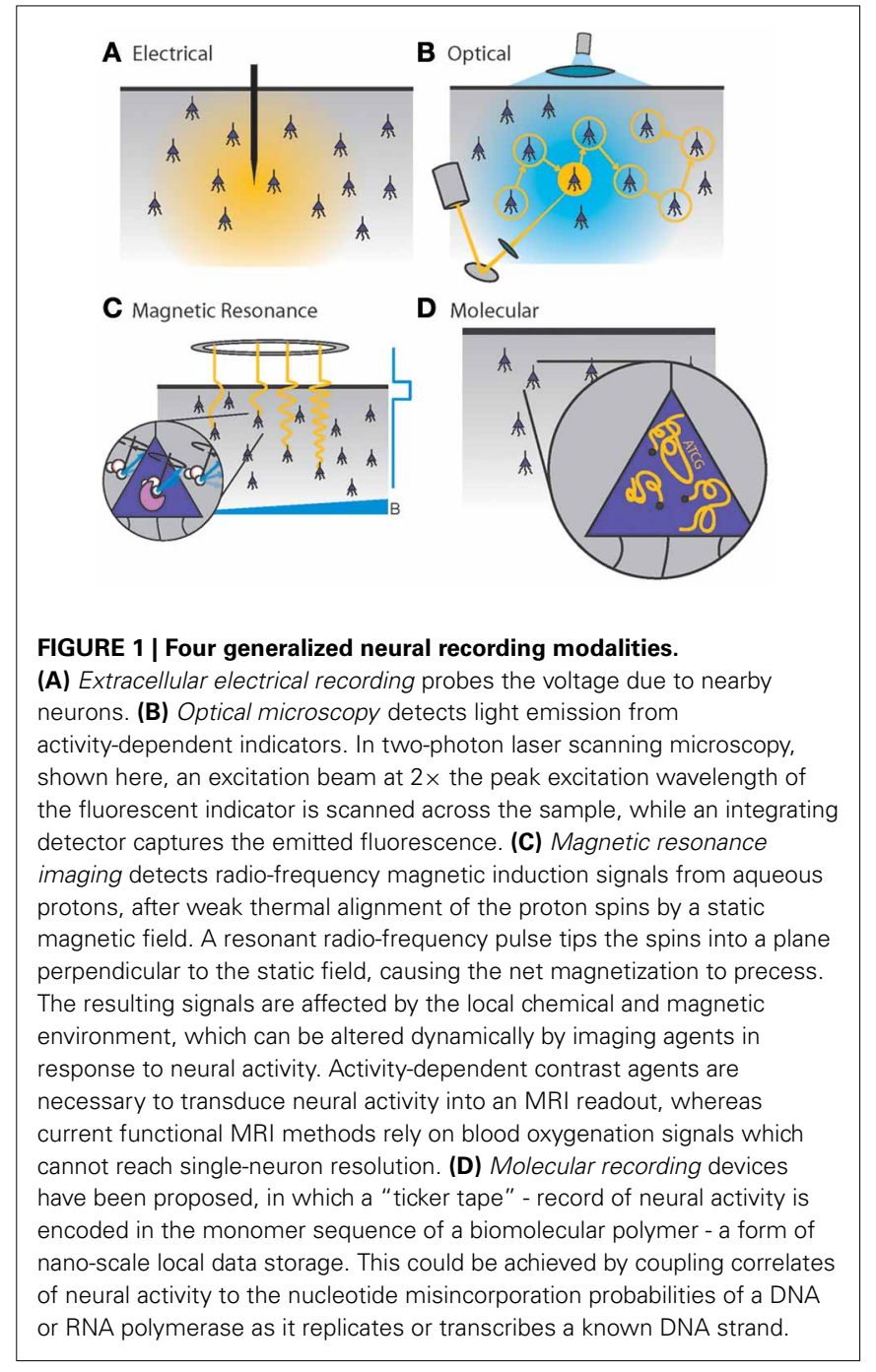

This corresponds roughly to one neuron per $22 \mu \mathrm{m}$ voxel. The density of cortical synapses, on the other hand, approaches $10^{9} / \mathrm{mm}^{3}$, i.e., one synapse per $1 \mu \mathrm{m}^{3}$ voxel. For comparison, the human brain has roughly $8 \times 10^{10}$ neurons (Azevedo et al., 2009) in a volume of $1200 \mathrm{~cm}^{3}$ (Allen et al., 2002).

The human brain consumes $\sim 15 \mathrm{~W}$ of power (performing, at synapses, a rough equivalent of at least $10^{17}$ floating point computational operations per second on that power budget, according to one definition (Sarpeshkar, 2010), although the analogy with digital computers should not be taken literally). Because power consumption scales approximately linearly with the number of neurons (Herculano-Houzel, 2011), the mouse brain is expected to utilize $\sim 15 \mathrm{~mW}$. For comparison, the metabolic rate of the $\sim 20-30$ g mouse is $\sim 200-600 \mathrm{~mW}$ depending on its degree of physical activity (Speakman, 2013).

\subsection{NEURAL ACTIVITIES}

Action potentials (spikes) last $\sim 2 \mathrm{~ms}$. The rate of neuronal spiking is highly variable. Some authors have assumed an average rate of $5 \mathrm{~Hz}$ (Sarpeshkar, 2010; Harris et al., 2012), but certain neurons spike at $500 \mathrm{~Hz}$ or faster (Gittis et al., 2010), while many 
neurons spike much more slowly. For example, cerebellar granule cells, which make up half of the neurons in the brain, have spontaneous firing rates of $\sim 0.5 \mathrm{~Hz}$ (Chadderton et al., 2004). In neocortex, one analysis estimated 0.16 spikes per second per neuron (in primate) as energetically sustainable (Lennie, 2003). There may be as much as a two-fold change in metabolism and hence firing rate across brain states (Howarth et al., 2012). Certain neurons (possibly up to $90 \%$ for some neuron types in some brain areas) may be effectively silent (Shoham et al., 2006; Barth and Poulet, 2012), e.g., spiking less than once every 10 s. Some studies have attempted to measure the distribution of neural firing rates in various cortical areas (as opposed to just the average rate), and have observed that these distributions are often long-tailed: a small minority of the neurons fires a majority of the spikes (Shafi et al., 2007; Hromádka et al., 2008; O’Connor et al., 2010; Roxin et al., 2011).

While these estimates of typical firing rates are useful numbers to have in mind, in the below we aim to sample all neurons at $1 \mathrm{kHz}$ rates (or higher for techniques requiring observation of detailed spike waveforms). This choice is informed by several factors. First, measuring spike timing with millisecond precision is relevant for understanding network function, due to the possibilities for timing codes, spike-timing dependent plasticity mechanisms, and other effects relying on temporally-precise spiking patterns (Markram et al., 2011; Babadi and Abbott, 2013; Gire et al., 2013; Taillefumier and Magnasco, 2013). In this regard, it is also important for a recording method to maintain precise temporal phasing between measurements at different brain locations: activity measurements should be locked to precise global clocks, perhaps with a tolerable phase imprecision between any two measurements in the range of $\frac{1}{2 \pi} \times 1 \mathrm{~ms} \approx$ 100-200 ms. Furthermore, the activities of neurons can be highly correlated locally or across large networks (Schneidman et al., 2006), suggesting that local activity sensors may be subjected to high instantaneous total firing rates due to simultaneously-active neurons.

\subsection{ABSORPTION AND SCATTERING OF RADIATION}

All existing methods of neural recording utilize electromagnetic waves, from the near-DC frequencies of wired electrical recordings $(\sim 1 \mathrm{kHz})$ to the radio-frequencies of wireless electronics and $\mathrm{fMRI}(\mathrm{MHz}-\mathrm{GHz})$ to visible light in optical approaches $(\sim 500 \mathrm{THz})$. These electromagnetic waves are attenuated in brain tissue by absorption and scattering. As an approximation to the electromagnetic absorption by brain tissue, we treat the absorption by water, the brain's main constituent $(68-80 \%$ by mass in humans Dobbing and Sands, 1973; Fatouros and Marmarou, 1999). At visible and near-IR wavelengths, scattering dominates absorption: absorption lengths are in the $\sim 1 \mathrm{~mm}$ range, while scattering lengths are $\sim 25-200 \mu \mathrm{m}$ (Wilt et al., 2009). The combined effect of absorption and scattering is measured by the attenuation length, the distance over which the signal strength is reduced by a factor of $1 / e$ along a path. Figure 2 shows the absorption length of water (Kou et al., 1993), and the attenuation length in a Mie scattering model from Horton et al. (2013) intended to approximate the scattering properties of cortical tissue [and see (Gabriel et al., 1996) for tissue skin depth

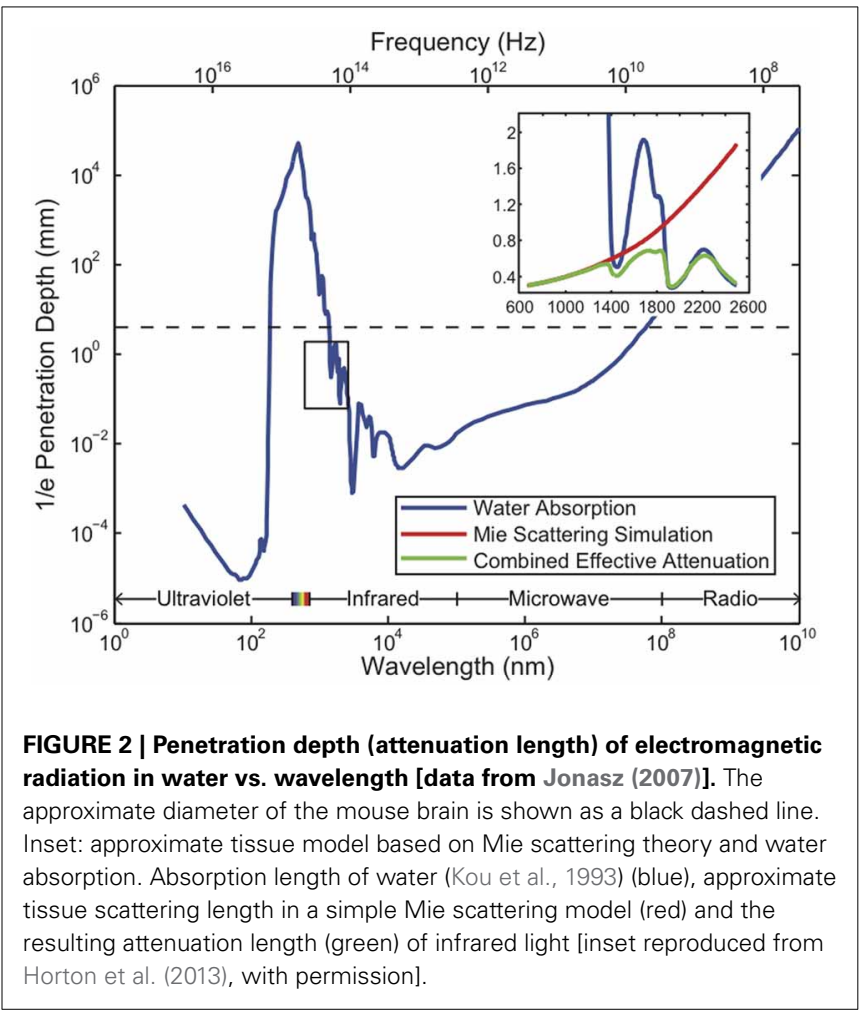

measurements in the $10 \mathrm{~Hz}$ to $100 \mathrm{GHz}$ range]. This gives a preliminary indication of which wavelengths can be used to measure deep-brain signals with external detectors. Note that the attenuation length is only one of several relevant metrics: for example, scattering not only causes signal attenuation, but also causes noise and impairs signal separation, so the magnitude of the scattering is a key figure of merit.

\section{CHALLENGES FOR BRAIN ACTIVITY MAPPING}

Any activity mapping technology must extract the required information without disrupting normal neuronal activity. As such, we consider three primary challenges: spatiotemporal resolution and informational throughput, energy dissipation and volume displacement.

\subsection{SPATIOTEMPORAL RESOLUTION AND INFORMATIONAL THROUGHPUT}

A sampling rate of $1 \mathrm{kHz}$ is necessary to capture the fastest trains of action potentials at single-spike resolution. A minimal data rate of $7.5 \times 10^{10}$ bits processed per second is then required to record 1 bit per mouse neuron at $1 \mathrm{kHz}$.

In electrical recording, higher sampling rates (e.g., $10-40 \mathrm{kHz}$ ) are often necessary to distinguish neurons based on spike shapes when each electrode monitors multiple neurons. More fundamentally, one bit per neuron sampling at $1 \mathrm{kHz}$ would likely not be sufficient to reliably distinguish spikes above noise: transmitting $\sim 10$ bit samples at $\sim 10 \mathrm{kHz}$ (full waveform) or $\sim 10-20$ bit time-stamps upon spike detection would be more realistic.

Conversely, it may be possible to locally compress measurements of a spike train before transmission. The degree of 
compressibility of neural activity data is related to the variability in the distribution of neural responses (e.g., such a distribution may be defined across time bins or repeated stimulus presentations) (Strong et al., 1998). In the blowfly Calliphora vicina, the entropy of spike trains has been measured to be up to $\sim 180 \mathrm{bit} / \mathrm{s}$, and the information about a stimulus encoded by a spike train was as high as $~ 90 \mathrm{bit} / \mathrm{s}$ (Strong et al., 1998). Extrapolating from fly to mouse, this would suggest that a compression factor of $5 \times-10 \times$ should be possible, relative to a 1000 bit/s raw binary sampling.

As a naïve estimate of the entropy as a function of firing rate, one can write the entropy $H$ in bit/s, assuming $1 \mathrm{~ms}$ long spikes and $f=1000 \mathrm{~Hz}$ sampling rate, as

$H \approx\left(-P_{\text {spike }} \cdot \log _{2}\left(P_{\text {spike }}\right)-\left(1-P_{\text {spike }}\right) \cdot \log _{2}\left(1-P_{\text {spike }}\right)\right) \cdot f$

where $P_{\text {spike }}$ is the probability of spiking during the sampling interval (average firing rate/f). For an average firing rate of $5 \mathrm{~Hz}$, $P_{\text {spike }}=0.005$ and $H=45 \mathrm{bit} / \mathrm{s}$, corresponding to a compression factor of $\sim 20 \times$. However, at $500 \mathrm{~Hz}$ average firing rate, $P_{\text {spike }}=0.5$ with $H \approx 1000 \mathrm{bit} / \mathrm{s}$, i.e., there is no compressibility. Therefore, compression could conceivably reduce the data transmission burden for activity mapping by 1-2 orders of magnitude, depending on the neurons and activity regimes under consideration. Note that these compressibility calculations have assumed that firing patterns are independent across cells; they represent the temporal compressibility of the spike train from each cell, treated individually. Patterns across cells could conceivably be compressed by a much larger amount, to the extent that there is redundancy between cells. Nevertheless, we use $1 \mathrm{bit} /$ neuron/ms or $100 \mathrm{Gbit} / \mathrm{s}$ as a "minimal whole brain data rate" in what follows. In many cases, this likely constitutes a lower bound on what is feasible in practice.

\subsection{ENERGY DISSIPATION}

Brain tissue can sustain local temperature increases $(\Delta T)$ of $\sim 2^{\circ} \mathrm{C}$ without severe damage over a timescale of hours. Indeed, changes of this magnitude may occur naturally in rats in response to varying activity levels (Wolf, 2008). Assuming that the brain is receiving a constant power influx $P_{\text {delivered }}$ and that the local thermal transport properties of mouse brains are similar to those of humans, we can approximate the temperature change in deepbrain tissue as a function of the applied power (Lazzi, 2005; Sotero and Iturria-Medina, 2011):

$$
\frac{\mathrm{d} T}{\mathrm{~d} t}=\left(P_{\text {delivered }}+P_{\text {metabolic }}-\rho \text { blood } C_{\text {blood }} f_{\text {blood }} \Delta T\right) / C_{\text {tissue }}
$$

where $P_{\text {metabolic }}=0.0116 \mathrm{~W} / \mathrm{g}$ is the power per unit mass of basal metabolism, $C_{\text {tissue }} \approx 3.7 \mathrm{~J} /(\mathrm{Kg}) \approx 0.88 \cdot C_{\text {water }}$ is the specific heat capacity of brain tissue, $\rho_{\text {blood }}=1.05 \mathrm{~g} / \mathrm{cm}^{3}$ is the density of blood, $C_{\text {blood }}=3.9 \mathrm{j} /(\mathrm{Kg})$ is the specific heat capacity of blood, $f_{\text {blood }}=9.3 \times 10^{-9} \mathrm{~m}^{3} / \mathrm{g} / \mathrm{s}$ is the volume flow rate of blood, and $\Delta T$ is the temperature difference between the brain tissue and the blood (at $37^{\circ} \mathrm{C}$ ). A steady-state temperature increase $(\mathrm{d} T / \mathrm{d} t=0)$ of $2^{\circ}$ corresponds to dissipation of $\sim 40 \mathrm{~mW}$ per $500 \mathrm{mg}$ mouse brain. Therefore, a recording technique should not dissipate more than $\sim 40 \mathrm{~mW}$ of power in a mouse brain at steady state.

This estimate of the power dissipation limit in mouse brains, based on such a simplified model of the brain's thermal transport mechanisms, is likely an under-estimate of the actual maximum steady-state power dissipation. Radiative heat loss was ignored here since infrared light emitted by deep-brain tissue is quickly re-absorbed by nearby tissue. We have also ignored cooling due to flows in the cerebrospinal ventricles (Smith and Zhu, 2010) and in the glymphatic system (Iliff et al., 2012). We have further assumed that conductive heat loss from the brain surface is negligible compared to the heat extracted volumetrically by blood flow. While this may hold true locally in deep brain voxels and over short timescales (e.g., $<1 \mathrm{~min}$ ), further work [e.g., a wholehead model (Sukstanskii and Yablonskiy, 2004; Lazzi, 2005)] is needed to define the true limits of sustained volumetric heat production by neural recording systems distributed throughout the mouse brain. Indeed, the characteristic length scale of temperature inhomogeneities in the brain is on the order of millimeters (Sukstanskii and Yablonskiy, 2006), whereas heat exchange with the flowing blood dampens the effects of local perturbations over longer length scales. For large brains, this means that sources and sinks of heat exert only local thermal effects; for a mouse brain on the scale of $<10 \mathrm{~mm}$, however, surface and volumetric effects likely combine to influence temperature changes at any site in the brain (Sukstanskii and Yablonskiy, 2007). Experimentally, increasing the temperature gradient at the brain surface, via a cranial window exposed to ambient air at $\sim 25^{\circ} \mathrm{C}$ (i.e., the common craniotomy technique used to access mouse neocortex), has been shown to dis-regulate brain temperature down to a depth of several millimeters (Kalmbach and Waters, 2012). For the above reasons, our estimates of the brain's capacity for heat dissipation should be treated only as first approximations.

Higher power levels, compared to the maximum steady state power, may be introduced into brains transiently. According to the above equation, if a neural recorder dissipates $\sim 40 \mathrm{~mW}$ per $500 \mathrm{mg}$ mouse brain, then the brain approaches the steady-state temperature in 2-3 min, making shorter experiments potentially feasible. This is in agreement with the estimate from Sukstanskii and Yablonskiy (2006) of a $\sim 1$ min time constant for brain temperature changes, as well as with experimental measurements showing similar time constants for temperature variations resulting from sustained neural stimulation (McElligott and Melzack, 1967; Trübel et al., 2005). Increasing convective heat loss from the brain by increasing blood flow (e.g., via increased heart rate) or cooling the brain (volumetrically or via its surface Sukstanskii and Yablonskiy, 2007), the blood, the cerebrospinal fluid (CSF), or the whole animal (Polderman, 2004), could increase the allowable transient or steady-state power dissipation.

There are also limits on the power density of radiation applied to brain tissue. For radio-frequency electromagnetic radiation, the specific absorption rate (SAR) limit on the power density exposed to human tissue is $\sim 10 \mathrm{~mW} / \mathrm{cm}^{2}$ (IEEE, 2006), while for ultrasound (which couples less strongly to dissipative loss mechanisms in tissue) the SAR limit is up to $72 \times$ higher (FDA, 2008). The power density limit for visible and near-IR light exposures 
are also in the $\sim 10-100 \mathrm{~mW} / \mathrm{cm}^{2}$ range for $\sim 1 \mathrm{~ms}$ long exposures, decreasing as the exposure time lengthens [based on the IEC 60825 formulas (IEC, 2007)].

High local power dissipation (transient or steady-state) can modify the electrical properties of excitable membranes, altering neuronal activity patterns. For example, heating of cell membranes and of the surrounding solution by millisecondlong optical pulses leads to changes in membrane electrical capacitance mediated by the ionic double layer (Shapiro et al., 2012). Slower temperature changes (on a scale of seconds) resulting from RF radiation lead to accelerated ion channel and transporter kinetics (Shapiro et al., 2013). Both of these effects are appreciable when the temperature changes are on the order of $1-10^{\circ} \mathrm{C}$.

For comparison with current practice, common guidelines for chronic heat exposure from biomedical implants (Wolf, 2008) use upper limits of $2^{\circ} \mathrm{C}$ temperature change, $40 \mathrm{~mW} / \mathrm{cm}^{2}$ heat flux from the surface of implanted brain machine interface (BMI) hardware, and an SAR limit of

$$
\frac{\sigma E^{2}}{2 \rho}<1.6 \mathrm{~mW} / \mathrm{g}
$$

for electromagnetic energy absorbed by tissue, where $E$ is the peak electric field amplitude of the applied radiation, $\sigma \approx 0.18 \mathrm{~S} / \mathrm{m}$ is the electrical conductivity of grey matter and $\rho \approx 1 \mathrm{~g} / \mathrm{cm}^{3}$ is the tissue density (Lazzi, 2005) (this corresponds to an irradiance of $\varepsilon_{0} c E^{2} / 2 \approx 2.4 \mathrm{~mW} / \mathrm{cm}^{2}$ ). A 96-channel BMI system demonstrated in living brains had dissipated areal power density approaching $40 \mathrm{~mW} / \mathrm{cm}^{2}$ (Rizk et al., 2009).

\subsection{SENSITIVITY TO VOLUME DISPLACEMENT}

To prevent damage to the brain, we assume that a recording technique should not displace $>1 \%$ of the brain's volume. The appropriate damage threshold is not yet established, however, so this constitutes a first guess. It is possible to insert large numbers of probes throughout multiple brain areas without compromising function. In rats, 96 electrodes of $50 \mu \mathrm{m}$ diameter were simultaneously inserted across four forebrain structures (cortex, thalamus, hippocampus and putamen) (Ribeiro et al., 2004). In rhesus macaque, 704 electrodes of diameter $50 \mu \mathrm{m}$ and average depth $2.5 \mathrm{~mm}$ were chronically implanted in cortex (Nicolelis et al., 2003). Note, however, that the total volume displacement in these experiments was below $0.1 \%$, and below $0.01 \%$, respectively. Furthermore, these studies used a low density of electrodes. Thus, detailed limits on the amount and density of inserted material are unknown.

Furthermore, the nature of the volume displacement is important - sheets of instrumentation that sever long-range connectivity, for example, would disrupt normal brain function regardless of the degree of volume displacement. Conversely, higher volume displacement might be possible if introduced gradually, or during early development, insomuch as the brain can adapt without disrupting natural computation. One important consideration in this regard would be the disruption of blood circulation by inserted material; a high density of implanted material in a brain region could cause stroke due to widespread vascular damage. Recent studies have defined in microscopic detail the complete vascular network of the mouse cortex using high-throughput histology (Blinder et al., 2013); this type of information could be used to enumerate key vascular pathways which could be spared from damage. To apply this in a particular animal, however, would require a nondestructive method to image the vasculature at a similar resolution; otherwise, only a broad statistical view can be obtained, since the detailed vascular geometry will vary from animal to animal.

Secondary effects like glial scarring may also pose obstacles to the long-term implantation of large numbers of probes (Polikov et al., 2005; Ward et al., 2009), although methods are being developed to alleviate this (Reichert et al., 2008; Reichert, 2010; Taub et al., 2012). In the context of electrical recording, the impact of glial scarring may vary depending on geometry. For example, the recording sites at the tip of a Utah or Duke multi-electrode array are typically viable in chronic recordings of up to 18 months in primates (Nicolelis et al., 2003; Suner et al., 2005), whereas in array formats with multiple electrodes along each shaft, such as the Michigan array, chronic recordings of up to 4 months have been reported in rats (Vetter et al., 2004). Differences in recording lifetime may be due to differences in the pattern of glial encapsulation of the contacts.

\section{EVALUATION OF MODALITIES}

We next evaluate neural recording technologies with respect to the above challenges, using the mouse brain as a model system. Table 1 lists the modalities studied, the assumptions made, the analysis strategies applied, and the conclusions derived.

\subsection{ELECTRICAL RECORDING}

In the oldest strategy for neural recording, an electrode is used to measure the local voltage at a recording site, which conveys information about the spiking activity of one or more nearby neurons. The number of recording sites may be smaller than the number of neurons recorded since each recording site may detect signals from multiple neurons. As a note for practitioners, we use the term "electrode" interchangeably with the terms "recording site" or "contact", meaning a point-like voltage sensing node: many multi-electrode arrays in common use (e.g., the Duke and Utah arrays) are conductive only at the tip, whereas other designs (such as the Michigan array) have multiple contacts along the shaft. Each shaft in a Michigan array would thus constitute multiple "electrodes" or "recording sites" in our parlance. Traditional electrical recording techniques keep active devices such as amplifiers outside the skull and therefore do not pose a heat dissipation challenge; this may change if amplifiers are brought closer to the signal sources to reduce noise.

Slowly varying (e.g., $<300 \mathrm{~Hz}$ ) extracellular potentials (LFPs) (Buzsáki et al., 2012; Reimann et al., 2013) on the order of 0.1-1 mV, and fields (Anastassiou et al., 2010) on the order of $1-10 \mathrm{mV} / \mathrm{mm}$, are generated by neural activity. While LFPs can be filtered from the higher-frequency signals associated with extracellular voltage spikes, these and other effects necessitate maintaining precise potential references (i.e., ground levels) for voltage measurements distributed widely across the brain. 
Table 1 | Summary of modalities, models, assumptions and conclusions.

\begin{tabular}{|c|c|c|c|}
\hline Modality & Analysis Strategy & Assumptions & Conclusions \\
\hline $\begin{array}{l}\text { Extracellular } \\
\text { electrical recording }\end{array}$ & $\begin{array}{l}\text { Compute minimal number of } \\
\text { recorders based on max distance } \\
\text { from recorder to recorded neuron } \\
\text { Compute channel capacity limits } \\
\text { to spike sorting }\end{array}$ & $\begin{array}{l}\text { Decay profile of extracellular voltage } \\
\text { Approximate noise levels at recording } \\
\text { site }\end{array}$ & $\begin{array}{l}\text { Maximum recording distance } r_{\max } \approx 100-200 \mu \mathrm{m} \\
\text { from electrode to neuron measured } \\
\sim 10^{5} \text { recording sites are required per mouse } \\
\text { brain at current noise levels assuming perfect } \\
\text { spike sorting } \\
\sim 10^{6} \text { recording sites are required at current noise } \\
\text { levels at the physical limits of spike sorting } \\
\sim 10^{7} \text { recording sites are required using current } \\
\text { spike sorting algorithms }\end{array}$ \\
\hline
\end{tabular}

Implanted electrical Compute power dissipation of recorders electronic devices that digitally sample neuronal activity
Physical limit: $k_{\mathrm{B}} T \ln (2) /$ bit erased Practical limit: $\sim 10 k_{\mathrm{B}} T /$ bit processed Current $\mathrm{CMOS}$ digital circuits: $>10^{5} \mathrm{kBT} /$ bit processed

Requires 2-3 orders of magnitude increase in the power efficiency of electronics relative to current devices to scale to whole-brain simultaneous recordings

Minimalist architectures could be developed to reduce local data processing overhead

Transmission at optical or near-optical frequencies is needed to achieve sufficient single-channel data rates using electromagnetic radiation. Radio-frequency (RF) electromagnetic transmission of whole-brain activity data draws excessive power due to bandwidth constraints Bandwidth cannot be split over multiple independent RF channels, but IR light or ultrasound may allow spatial multiplexing

\begin{tabular}{ll}
\hline Optical imaging & Relate the scattering and \\
& absorption lengths of optical \\
& wavelengths in brain tissue to \\
& signal-to-noise ratios for optical \\
& imaging
\end{tabular}

Approximate values of scattering and absorption lengths as a function of wavelength

Light scattering imposes severe constraints, but strategies exist which could negate the effects of scattering, such as implantable optics, infrared indicators, signal modulation, and online inversion of the scattering matrix

\begin{tabular}{lll}
$\begin{array}{l}\text { Multi-photon } \\
\text { optical imaging }\end{array}$ & $\begin{array}{l}\text { Compute minimum total } \\
\text { excitation light power to excite } \\
\text { multi-photon transitions from } \\
\text { indicators within each neuron in } \\
\text { every imaging frame }\end{array}$ & $\begin{array}{l}\text { Approximate values of multi-photon } \\
\text { cross-sections } \\
\text { Pulse durations similar to those } \\
\text { currently used in multi-photon imaging }\end{array}$ \\
\hline $\begin{array}{l}\text { Beam scanning } \\
\text { microscopies }\end{array}$ & $\begin{array}{l}\text { Calculate device and indicator } \\
\text { parameters necessary for fast } \\
\text { beam repositioning and signal } \\
\text { detection }\end{array}$ & $\begin{array}{l}\text { Fast optical phase modulators could } \\
\text { reposition beams at } \sim \text { GHz switching } \\
\text { rates } \\
\text { Fluorescence lifetimes in the }\end{array}$ \\
& & $0.1-1.0 \mathrm{~ns}$ range
\end{tabular}

Magnetic resonance imaging
Calculate spatial and temporal resolution of MRI based on spin relaxation times and spin diffusion
Proton MRI using tissue water Approximate $T_{1}$ and $T_{2}$ relaxation times and self-diffusion times for tissue water
Whole-brain multi-photon excitation will over-heat the brain except in very short experiments, unless ultra-high-cross-section indicators are used
Beam repositioning time limits the speed of current systems but these are far from the physical limits

Fluorescence lifetimes of indicators constrain design of ultra-fast scanning microscopies

Proton MRI is limited by the $T_{1}$ relaxation time of water to $\sim 100 \mathrm{~ms}$ temporal resolution and by the self-diffusion of water to spatial resolutions of $\sim 40 \mu \mathrm{m}$. $T_{1}$ pre-mapping could allow $T_{2}$ contrast on a $\sim 10 \mathrm{~ms}$ timescale. Achieving these limits for functional imaging requires going beyond BOLD contrast

Attenuation of ultrasound by brain tissue and bone may be prohibitive at the $\sim 100 \mathrm{mHz}$ frequencies needed for single-cell resolution ultrasound imaging Ultrasound may be viable for spatially multiplexed data transmission from embedded devices (Seo et al., 2013) 
Table 1 | Continued

\begin{tabular}{|c|c|c|c|}
\hline Modality & Analysis Strategy & Assumptions & Conclusions \\
\hline $\begin{array}{l}\text { Molecular } \\
\text { recording }\end{array}$ & $\begin{array}{l}\text { Compute metabolic load and } \\
\text { volume constraint for rapid } \\
\text { synthesis of large nucleic acid } \\
\text { polymers } \\
\text { Evaluate temporal resolution in } \\
\text { simulated experiments using } \\
\text { kinetic models (Glaser et al., 2013) }\end{array}$ & $\begin{array}{l}\text { Polymerase biochemical parameter } \\
\text { ranges } \\
\text { Metabolic requirements of genome } \\
\text { replication }\end{array}$ & $\begin{array}{l}\text { Molecular recording devices appear to fall within } \\
\text { physical limits but their development poses } \\
\text { multiple major challenges in synthetic biology } \\
\text { Synchronization or time-stamping mechanisms } \\
\text { are required for temporal resolution to approach } \\
\text { the millisecond scale }\end{array}$ \\
\hline
\end{tabular}

\subsubsection{Spatiotemporal resolution}

4.1.1.1. Limits assuming perfect spike sorting. We begin with an idealized estimate of the number of electrodes required to record from the entire mouse brain, neglecting the difficulty of assigning observed spikes to specific cells (spike sorting), and focusing only on what is needed to detect spikes from every neuron on at least one electrode. The key variable here is the maximum distance between an extracellular electrical recorder and a neuron from which it records spikes. In a first approximation, this is determined by two factors: the decay of the signal with distance from the spiking neuron and the background noise level at the recording site. We assume that for an electrode to reliably detect the signal from a given neuron, the magnitude of that neuron's signal must be larger than the electrode's noise level. Note, however, that knowledge of spike shape distributions could potentially be used to extract low-amplitude spikes from noise.

The peak signals of spikes from neurons immediately adjacent to an electrode are in the $0.1-1.0 \mathrm{mV}$ range and scale roughly as $e^{-r / r_{0}}$, where $r$ is the distance from the cell surface and the $1 / e$ falloff distance, $r_{0}$, has been experimentally measured at $\sim 28 \mu \mathrm{m}$ in both salamander retina (Segev et al., 2004) and cat cortex (Gray et al., 1995), and computed at $\sim 18 \mu \mathrm{m}$ in a biophysically realistic simulation (Gold et al., 2007; Anastassiou et al., 2013). However, this decay is strongly influenced by the detailed geometry of neuronal currents and the properties of the extracellular space [e.g., its inhomogeneity, which may lead to a frequency-dependent falloff of the extracellular potential (Bédard et al., 2004)], making analytical calculation of the decay rate difficult (at large distances, a much slower $1 / r^{2}$ dipole falloff is expected).

Several sources of background noise enter the recordings. Johnson noise, which arises from thermal fluctuations in the electrode, is

$$
V_{\text {johnson }}=\left(4 k_{\mathrm{B}} T Z \mathrm{BW}\right)^{1 / 2}
$$

which for physiological temperature, electrodes of impedance $Z=0.5 \mathrm{~m} \Omega$, and $\mathrm{BW}=10 \mathrm{kHz}$ bandwidth is $V_{\text {johnson }} \approx 9 \mathrm{mv}$. The recordings are also affected by interference from other neurons, which has been reported to exceed the Johnson noise, and is non-stationary due to changes in the cells' firing properties (Sahani, 1999). The noise and interference from these sources realistically produces $>10-20 \mu \mathrm{V}$ of voltage fluctuations (Camuñas Mesa and Quian Quiroga, 2013). Current recording setups thus have signal to interference-plus-noise ratios (SINRs) of $<100$, where the SINR is defined as the ratio of the peak voltage from immediately adjacent neurons to the voltage fluctuation floor of the electrode.

A limit on the maximum recording distance is the distance at which the signal from the farthest neuron falls below the noise floor, $r_{\max } \approx r_{0} \ln (\mathrm{SINR})$. For SINR $\approx 100, r_{\max } \approx 130 \mu \mathrm{m}$. For comparison, recent experimental data from multi-site silicon probes has shown few detectable neurons beyond $\sim 100 \mu \mathrm{m}$ and none detectable beyond $160 \mu \mathrm{m}$ (Du et al., 2011). Recordings in the hippocampal CA1 region could not detect spikes from cells farther than $140 \mu \mathrm{m}$ from the electrode tip (Henze et al., 2000), even after averaging over observations triggered on an intracellularly recorded spike; in hippocampus, this corresponds to a detection volume containing approximately 1000 neurons (Buzsáki, 2004). Furthermore, in many studies (in monkeys, rats and mice) using multi-electrode arrays with 150-300 $\mu \mathrm{m}$ interelectrode spacings, no neuron is seen by more than one electrode (Wessberg et al., 2000; Carmena et al., 2003; Jin and Costa, 2010; Koralek et al., 2012).

Due to the steep local falloff, even improving the SINR by a factor of 10 only extends the maximal recording distance to $r_{\max } \approx 190 \mu \mathrm{m}$. Assuming packing of the brain into equal sized cubes of side length $d=\frac{2 \sqrt{3}}{3} r_{\max } \approx 150 \mu \mathrm{m}$ gives $N>13,0000$ electrodes for whole brain recording using recording sites with $r_{\max } \approx 130 \mu \mathrm{m}$. Note that $N$ varies as the third power of $r_{\max }$ and is therefore highly sensitive to variations in the assumed maximal recording distance; the number of required recorders can range from 38,000 to 210,000 as $r_{\max }$ varies from 190 to $110 \mu \mathrm{m}$.

These calculations, by assuming perfect spike sorting, greatly underestimate the required number of electrodes in practice. First, signals from the weakest cells are far weaker than those from the strongest cells and the signals from some cells decay much faster than others (Gray et al., 1995). Second, because of neuronal synchronization, the local noise produced by nearby neurons may sometimes be large. Third, spike waveforms can vary over the course of a recording session (Fee et al., 1996; Stratton et al., 2012). Finally, with many neurons per electrode or at high firing rates, spikes from detectable neurons will often temporally overlap, making spike sorting difficult.

4.1.1.2. Limits from spike sorting. The previous calculations have assumed that any spike which is visible above the noise on at least one electrode can be detected and correctly assigned to a particular cell, i.e., that the problem of spike sorting can be solved perfectly. However, perfect spike sorting is far beyond 
current algorithmic capabilities and in fact may not be possible in principle.

To achieve the scenario described above, with $N=13,0000$ recording sites per mouse brain, would require each electrode to sort spikes from all $\frac{4}{3} \pi r_{\max }^{3} \rho_{\text {neurons }}$ neurons in a sphere of radius $r_{\max } \approx 130 \mu \mathrm{m}$ surrounding the recording site, where $\rho_{\text {neurons }} \approx 92,000 / \mathrm{mm}^{3}$ is the density of neurons. This assigns $\sim 800$ neurons to a single electrode. Roughly half (i.e., 400) of these neurons will lie at $>100 \mu \mathrm{m}$ distance from the electrode, and their signals on the electrode will therefore have voltage SINRs of $<100 e^{-100 \mu \mathrm{m} / 28 \mu \mathrm{m}} \approx 2.8$, assuming as above that extracellular spike amplitudes decay exponentially in space.

Electrical recording can be viewed as a data transmission problem, with the electrode playing the role of a communication channel (see section 4.4). According to the Shannon Capacity Theorem (Cover and Thomas, 2006), the information capacity $C$ of a single analog channel (with additive white Gaussian noise) is

$$
C=\mathrm{BW} \log _{2}(1+S / N)
$$

where BW is the bandwidth, $S$ is the signal power (proportional to the square of the voltage), and $N$ is the noise power. Here the bandwidth is $\mathrm{BW} \approx 10 \mathrm{kHz} / \mathrm{s}$, and the ratio of peak signal power to noise power of a single spike for the outer 400 cells is no more than $2.8^{2}$, or $0.5 \times 2.8^{2}$ using the RMS signal power instead of the peak. With 400 cells emitting $2 \mathrm{~ms}$ spikes at $5 \mathrm{~Hz}$, there will be an average of 4 cells spiking at a time, for $S / N \approx 0.5 \times 4 \times$ $2.8^{2} \approx 15.7$ counting the signal power from all the spikes. The channel capacity is then $C \approx 40 \mathrm{kbit} / \mathrm{s}$. This represents the maximum amount of information (e.g., about which neuron spiked when) that the population of spiking neurons can transmit via the electrode which measures them. To transmit uniquely identifiable signals from all 400 neurons at millisecond temporal precision, however, requires $1 \mathrm{kbit} / \mathrm{s} \times 400=400 \mathrm{kbit} / \mathrm{s}$, which is $>10 \times$ greater than the channel capacity and is therefore not achievable. Even with optimal temporal compression of $\sim 5 \mathrm{~Hz}$ spikes (see section 2 ), we would need to transmit $\sim 400 / 20=20 \mathrm{kbit} / \mathrm{s}$, which is strictly less than the channel capacity and thus possible in principle, but barely so. Furthermore, the channel capacity given here is an overestimate, since 2.8 is an upper bound on the SINR of the outer cells. On the other hand, note that the use of a nominal $5 \mathrm{~Hz}$ average firing rate here (in the estimates of signal to noise ratio and of temporal compressibility) greatly oversimplifies the distribution of firing rates across neurons, as discussed in section 2 above, so this analysis can only be treated as a first approximation.

Based on these rough estimates, perfect spike sorting may not be possible at $\sim 800$ neurons per electrode, in a sphere of radius $130 \mu \mathrm{m}$ surrounding a recording site, and at the noise levels typical of current electrodes. In essence, there may not be enough room on the electrode's voltage trace to discriminate such a large number of weak, noisy signals. Note that these information-theoretic limits still apply even if it is possible to resolve temporally overlapping spikes. In fact, the channel capacity is what ultimately limits the ability of a spike sorting algorithm to resolve such overlapping spikes.

To see the regime in which spike sorting becomes feasible, suppose that each electrode is only responsible for spike sorting from the population of $\sim 100$ neurons nearest to the electrode, i.e., in a sphere of radius $r \approx 64 \mu \mathrm{m}$, assuming the $92,000 / \mathrm{mm}^{3}$ cell density from mouse cortex. The outermost $50 \%$ of these neurons are then positioned $>50 \mu \mathrm{m}$ from the recording site. For these outermost 50 neurons, the voltage SINR is $<100 e^{-50 \mu \mathrm{m} / 28 \mu \mathrm{m}} \approx 17$ and $S / N<0.5 \times 17^{2} \times(2 \mathrm{~ms} \times 5 \mathrm{~Hz} \times 50) \approx 72.3$. The channel capacity is therefore $<62 \mathrm{kbit} / \mathrm{s}$, whereas $50 \mathrm{kbit} / \mathrm{s}$ is needed for signal transmission from 50 neurons without temporal compression versus $\sim 2.5 \mathrm{kbit} / \mathrm{s}$ with temporal compression. Even 100 neurons per electrode may therefore still be close to the limits of information transmission through the noisy channel corresponding to a single electrode.

In practice these limits are likely to be highly optimistic, since the set of spikes emerging from a neuronal population is far from an optimally designed code from the perspective of multiplexed signal transmission through a voltage-sensing electrode: the waveforms for different neurons are similarly-shaped rather than orthogonal, the spikes emitted by a given neuron vary somewhat in amplitude and exhibit shape fluctuations (signaldependent noise), and it is not known in advance what the characteristic signal from each neuron looks like (or even how many neurons there are).

Indeed, current practice is far from the above informationtheoretic limits. At present, spike sorting algorithms operating on data from large-scale (250-500 electrodes), densely spaced $(\sim 30 \mu \mathrm{m}), 2 \mathrm{D}$ multi-electrode arrays can reliably identify and distinguish spikes from nearly all of the 200-300 retinal ganglion cells (Marre et al., 2012; Pillow et al., 2013) in a small patch of retina, and can also infer approximate cell locations through spatial triangulation of spike amplitudes. This represents a roughly $1: 1$ ratio of cells to electrodes. Electrodes with up to 4 single units can be found in chronically implanted multielectrode arrays (in both mouse and primate) (Nicolelis et al., 2003; Costa et al., 2004), where the electrodes are sparse, although the average yield of cells per electrode is closer to $1: 1$; if only electrodes with at least one cell are counted, the average rises to $\sim 1.5-1.7$ cells per electrode. Optimistically, simulations of neural activity suggest that 5-10 neurons per electrode may be distinguishable using current spike sorting algorithms (Sahani, 1999; Pedreira et al., 2012; Camuñas Mesa and Quian Quiroga, 2013). A limit of $\sim 10$ neurons per electrode would imply $N=7.5 \times 10^{6}$ electrodes to record from all neurons in the mouse brain, which could be accomplished by positioning recording sites on a cubic lattice with $\sim 40 \mu \mathrm{m}$ edge length.

Future algorithmic improvements could enable sorting from more than $\sim 10$ cells per electrode, but this becomes increasingly challenging. One simple estimate of a reasonable practical limit, for the regime of many neurons per electrode, would be the largest number of neurons that can be sorted without requiring the frequent resolving of temporally overlapping spikes: if the average neuron fires at $\sim 5 \mathrm{~Hz}$ and spikes last $\sim 2 \mathrm{~ms}$, then at most roughly 100 neurons per electrode can be sorted without requiring overlaps to be resolved. Note that while some present-day algorithms can successfully resolve overlapping spikes (Segev et al., 2004; Ge et al., 2011; Prentice et al., 2011; Marre et al., 2012; Pillow et al., 2013), they typically do so only in the case where electrodes are densely spaced and any given spike appears on many electrodes, 
such that spatial information can be used to resolve the overlap. Resolving overlaps when spikes appear on only one or a few channels is more difficult due to noise and spike-shape variation.

Overall, $\sim 100$ cells per electrode may be taken as a rough estimate of the limits of spike sorting, and would imply $N=750,000$ electrodes and an edge spacing of $\sim 80 \mu \mathrm{m}$ if a cubic lattice of recording sites were used. However, we should not exclude the possibility of game-changers which could alter the nature of the recorded data to improve the available information. For instance, CCD cameras could be attached to multi-electrode arrays to aid in the identification and localization of cells, or directional information on the source of spikes could be obtained at each recording site, for example by measuring the directions of gradients in voltage. Systems that capture such additional information could circumvent the above information-theoretic limits and improve spike sorting.

\subsubsection{Volume displacement}

We require $<1 \%$ total volume displacement from $N$ recorders. Wires from each electrode must make it to the surface of the brain, which implies an average length $l \approx 4 \mathrm{~mm}$ for the mouse brain (depending on assumptions about the wiring geometry).

As a rough approximation, consider each recorder to produce a volume displacement associated with a single cylindrical wire, with length $l$ and radius $r$. Thus $r$ must satisfy

$$
\pi r^{2} l N_{\text {min,rd }}<0.01 V_{\text {brain }}
$$

Using $N_{\text {min,rd }}=21,0000$ or 38,000 recording sites (lower and upper limits from the perfect spike sorting case from above) and $l \approx 4 \mathrm{~mm}$ requires wires of radius $r_{\max } \approx 6.0 \mu \mathrm{m}$, or $2.5 \mu \mathrm{m}$, respectively. Alternatively, if $7.5 \times 10^{6}$ electrodes must be used (current spike sorting case from above), the required wire radius is $\sim 200 \mathrm{~nm}$. While these dimensions are readily achievable using lithographic fabrication, there would be a challenge to produce isolated wires of such dimensions at scale (perhaps suggesting the use of wire bundles). Still, volume constraints per se are unlikely to fundamentally limit whole-mouse-brain electrical recording even in the most pessimistic scenario.

Figure 3 illustrates the above considerations as a function of the electrode SINR.

\subsubsection{Implanting electrodes in the brain}

There are several technology options for introducing many electrodes into a brain. For example, flexible nanowire electrodes could, in theory, be threaded through the capillary network (Llinás et al., 2005). Capillaries are present in the brain at a density of $2500-3000 / \mathrm{mm}^{3}$ (Schmidt and Thews, 1989), which equates to one capillary per $73 \mu \mathrm{m}$, with each neuron lying within $\sim 200 \mu \mathrm{m}$ of a capillary (Loffredo and Lee, 2008). The minimum capillary diameter is as small as $3-4 \mu \mathrm{m}$, although the average diameter is $\sim 8 \mu \mathrm{m}$, comparable to the non-deformed size of the red blood cells (Freitas, 1999). Blocking a significant fraction of capillaries could lead to stroke or to unacceptable levels of tissue necrosis/liquifaction.

The cerebrospinal ventricles may also provide a convenient location for recording hardware. Furthermore, neural tissues

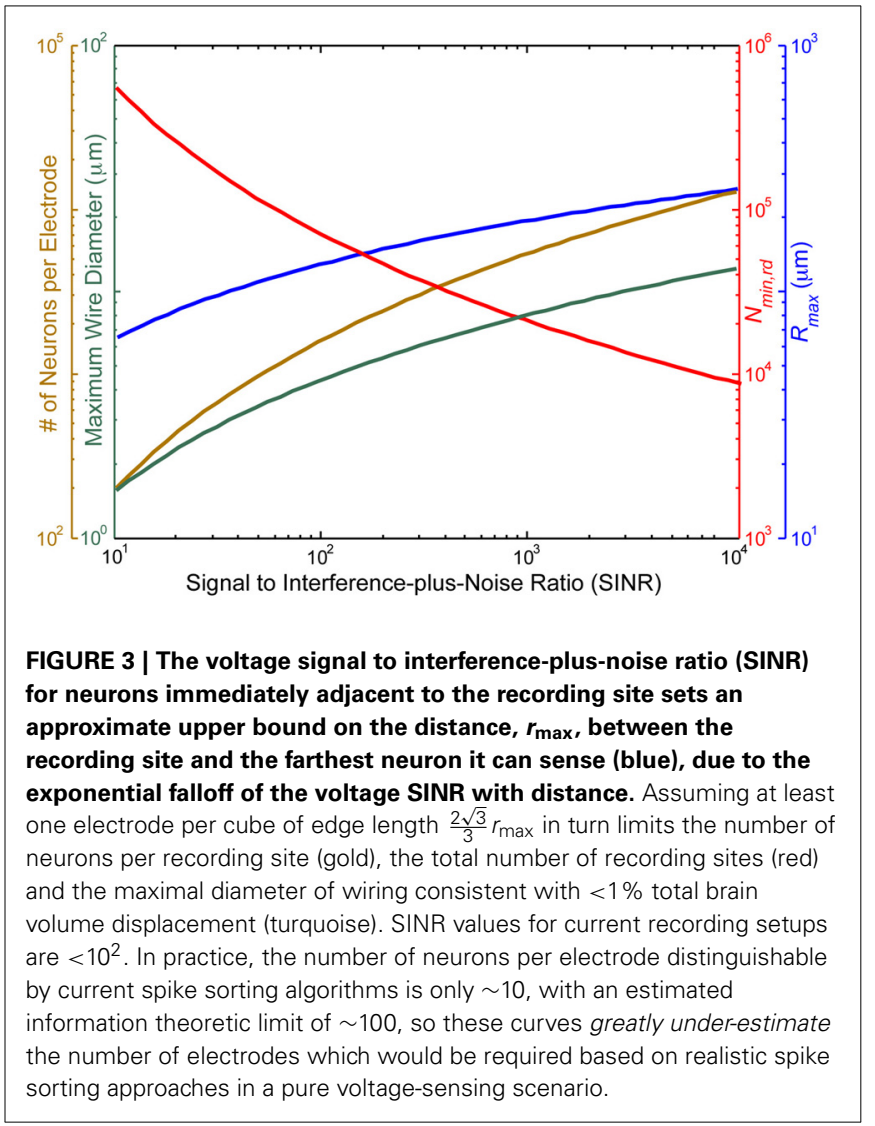

could be grown around pre-fabricated electrode arrays (Jadhav et al., 2012), or silicon probes arrays with many nano-fabricated recording sites per probe (Du et al., 2011) could be inserted into the brain.

Mechanical forces during insertion and retraction of silicon and tungsten microelectrodes from brain tissue have been measured in rat cortex at $\sim 1 \mathrm{mN}$ for electrodes of $\sim 25 \mu \mathrm{m}$ radius (Jensen et al., 2003). These forces are comparable to the Euler buckling force $F$ of a $2 \mathrm{~mm}$ long cylindrical tungsten rod of $r=$ $5 \mu \mathrm{m}$ radius

$$
F=\frac{\pi^{2} E I}{(K L)^{2}} \approx 1 \mathrm{mN}
$$

where $E=411 \mathrm{GPa}$ is the elastic modulus of tungsten, $I=$ $(\pi / 2) r^{4}$ is the moment of inertia of the wire cross-section, $L \approx$ $2 \mathrm{~mm}$ is the length of the wire, and $K$ is the column effective length factor which depends on the boundary conditions and is set to $K=1$ here for simplicity. This suggests that it may be possible to push structures of $<10 \mu \mathrm{m}$ diameter into brain tissue [see (Najafi and Hetke, 1990) for related calculations]. It might be advantageous to pull rather than push wires into the brain [e.g., using applied fields, or perhaps even cellular oxen (Weibel et al., 2005) to carry the wires], since the thinnest wires could withstand tension forces much higher than the compressive force at which they buckle (although there may also be ways to circumvent buckling, e.g., via rapid vibration). 


\subsubsection{Conclusions and Future Directions}

Electrical recording has the advantage of high temporal resolution, but the large number of required recording sites poses challenges for delivery mechanisms. Ongoing innovations in electrical recording that could be leveraged for dramatic scaling include the development of highly multiplexed probes, multilayer lithography for routing electrical traces, novel methods to implant large numbers of electrodes, smaller electrode impedances to reduce the Johnson noise, amplifiers with lower input-referred noise levels, spike sorting algorithms capable of handling temporally overlapping spikes and adaptively modeling the noise, and hybrid systems integrating electrical recording with implantable optics or other methods.

One challenge for a purely-electrical recording paradigm pertains to the ability to relate the measured electrical signals to specific cells within a circuit. As the set of neurons recorded by each electrode grows to encompass a large volume around the electrode, it will become more difficult to attribute the recorded spikes to particular neurons. Furthermore, given the complex geometries of neuronal processes, it is not obvious how to determine the spatial position or layout of a neuron from its electrical signature on a nearby electrode. A given electrode will be positioned near the axons or dendrites of some neurons, and near the cell bodies of other neurons, complicating data interpretation. If the spatial density of recording sites is increased such that many electrodes sample the same neuron, however, this could enable imaging of neuronal morphology and signal propagation via voltage signals across multiple electrodes (Bakkum et al., 2013). Currently, extracellular electrical recording also does not allow extraction of molecular information on the cells being recorded, although intracellular electrophysiological recording methods [e.g., (Kodandaramaiah et al., 2012)] might enable this for a limited number of cells.

\subsection{OPTICAL RECORDING}

Optical techniques measure activity-dependent light emissions from neurons, typically generated by fluorescent indicator proteins, although activity-dependent bioluminescent emissions are an emerging possibility. Current genetically encoded calcium indicators can only distinguish spikes below $\sim 50-100 \mathrm{~Hz}$ firing rates without averaging (Smetters et al., 1999) due to slow intra-molecular kinetics and indicator saturation at high firing rates, although significant improvements in speed are ongoing (Sun et al., 2013b). Intracellular calcium rises and drops can occur within $1 \mathrm{~ms}$ and 10-100 ms respectively (Higley and Sabatini, 2008), which sets the ultimate speed limit for calcium imaging. The field of genetically-encoded high-speed fluorescent voltage indicators is also advancing quickly (Barnett et al., 2012; Kralj et al., 2012; Akemann et al., 2013; Cao et al., 2013; Gong et al., 2013; Storace et al., 2013) and these may find particular use in monitoring sub-threshold events (Scanziani and Häusser, 2009).

\subsubsection{Spatiotemporal resolution}

4.2.1.1. Multiplexing strategies. For optical approaches, the light originating from the activity of each neuron must be separated from emissions originating from other points in the brain: this can be accomplished in many ways, leading to a variety of architectures for 3D imaging. Epi-fluorescence microscopy images a plane in the specimen (i.e., with depth of field DOF $=\frac{2 n \lambda}{N A^{2}}$, where $n$ is the refractive index, $\lambda$ is the wavelength and $N A$ is the numerical aperture of the imaging system Quirin et al., 2013) onto a spatially-resolved two-dimensional detector (e.g., a CCD camera). The focal plane is then scanned in order to reconstruct $3 \mathrm{D}$ images; because the entire $3 \mathrm{D}$ volume is illuminated during image acquisition, out-of-focus neurons cause background emissions. Light sheet imaging is similar to epi-flourescence imaging, except that only neurons near the focal plane are illuminated, reducing out of focus noise. Unfortunately, this requires transparent brains (Ahrens et al., 2013). Volumetric imaging can also be performed in a single snapshot using lightfield microscopes (Levoy et al., 2009; Broxton et al., 2013), which capture the directions of incoming light rays, trading in-plane resolution for axial resolution, or by using multi-focus microscopes (Abrahamsson et al., 2012). In multi-photon microscopy, non-linearities result in fluorescence excitation occurring only near the focal point of the excitation laser, which is scanned across the sample. In confocal scanning microscopy, only photons from a point of interest are measured due to geometric constraints (e.g., pinholes). Alternatively, 3D imaging can be performed via wavefront coding, which extends the depth of field by creating an axially-independent point-spread function using known optical aberrations, in combination with computational deconvolution (Dowski and Cathey, 1995). With a known 3D pattern of excitation light, wavefront coding can be applied to 3D fluorescence microscopy without scanning using a 2D detector array (Quirin et al., 2013). Emerging, alternative strategies rely on tagging emissions from different sources with distinguishable modulation patterns (Yin, 2006; Wu et al., 2006; Wang et al., 2012; Diebold et al., 2013; Ducros et al., 2013), or precisely controlling and tracking the timing of light emissions (Cheng et al., 2011). Optical techniques thus achieve signal separation by multiplexing spatially (e.g., direct imaging) or temporally (e.g., beam scanning), or often by a combination of the two.

While optics might seem to require a number of photodetectors comparable to the number of neurons (or a similar number of sampling events in the time domain, e.g., for scanning microscopies), new developments suggest ways of imaging with fewer elements. For example, compressive sensing or ghost imaging techniques based on random mask projections (Wakin et al., 2006; Tian et al., 2011; Studer et al., 2012; Sun et al., 2013a) might allow a smaller number of photodetectors to be used. In an illustrative case, an imaging system may be constructed simply from a single photodetector and a transmissive LCD screen presenting a series of random binary mask patterns (Huang et al., 2013), where the number of required mask patterns is much smaller than the number of image pixels due to a compressive reconstruction.

4.2.1.2. Effects of light scattering. Single-photon techniques limit imaging to a depth of a few scattering lengths at the excitation and emission wavelengths of activity indicators: up to $1-2 \mathrm{~mm}$ for certain infrared wavelengths (Horton et al., 2013; Kobat et al., 2009, 2011) vs. a few hundred microns for 
visible wavelengths (Wilt et al., 2009). Activity dependent dyes are currently available only in the visible spectrum; indicators operating in the infrared [see (Shcherbo et al., 2009; Filonov et al., 2011; Shcherbakova and Verkhusha, 2013) for far-red fluorescent proteins] could improve imaging depth.

Multi-photon excitation takes advantage of the deeper penetration of infrared light. Two or more infrared photons may together excite a fluorophore with an excitation peak in the visible range, leading to the emission of a visible photon. If only one neuron is illuminated with sufficient intensity to generate multiphoton excitation, all photons captured by the detector originate from that neuron, regardless of the scattering of the outgoing light. Hence, the emission pathway is limited less by scattering than by absorption. This has resulted in imaging at $>1 \mathrm{~mm}$ depth (Kobat et al., 2009, 2011; Horton et al., 2013).

There are at least five options for overcoming visible light scattering to enable signal separation from deep-brain neurons (Alivisatos et al., 2012, 2013):

1. Infrared light can excite multi-photon fluorescence in an excitation-scanning architecture.

2. Fluorophores with both excitation and emission wavelengths in the infrared could be developed.

3. By knowing the precise form of the scattering, it can be possible to correct for it. Emerging techniques based on beam shaping allow transmission of focused light through random scattering media by inverting the scattering matrix (Conkey et al., 2012). Because the scattering properties change over time, this must be done quickly, possibly faster than the imaging frame rate, necessitating high-speed wavefront modulation. This can currently be achieved with digital micro-mirror devices (DMDs), but not with the phase-only spatial light modulators (SLMs) that are used to prevent power losses in the excitation pathways for non-linear microscopies, although $\mathrm{GHz}$ switching of phase-only modulators appears feasible in principle (Alivisatos et al., 2013). High speed focusing through turbid media is also achievable using all-optical feedback in a laser cavity (Nixon et al., 2013), and it is even possible to measure the scattering matrix non-invasively (Chaigne et al., 2013) using a photo-acoustic technique, or via all-optical approaches based on speckle correlation (Bertolotti et al., 2012). Similar techniques are available for incoherent light (Katz et al., 2012). When using short optical pulses, scattering can lead to temporal distortions that degrade the peak light intensity at a focal spot. The $<100 \mathrm{fs}$ pulse durations used in two-photon microscopy, for example, are comparable to the time it takes light to travel $30 \mu \mathrm{m}$ in vacuum. Fortunately, wavefront shaping techniques can correct for scattering-induced temporal distortions as well (Katz et al., 2011; McCabe et al., 2011).

4. Light sources and/or detectors could be positioned close to the measured neurons, necessitating the use of embedded optical devices. This could be done using optical fiber (Mahalati et al., 2013) and/or waveguide (Zorzos et al., 2010, 2012) technologies, which are developing rapidly. For example, single-mode fiber cables can support $>1 \mathrm{~TB} / \mathrm{s}$ data rates (Ono and Yano, 1998; Bozinovic et al., 2013) with low light loss over hundreds of kilometers (Miya et al., 1979). It is possible to directly image through gradient index of refraction (GRIN) lenses (Murray and Levene, 2012) or optical fibers (Flusberg et al., 2005; Kang et al., 2010; Mahalati et al., 2013), which provides one way to multiplex multiple observed neurons per fiber.

5. Light emissions from distinct locations can be tagged with distinguishable time-domain modulation patterns, and the emission time-series for each source can later be decoded from the summed signal resulting from scattering (Wu et al., 2006; Yin, 2006; Cheng et al., 2011; Wang et al., 2012; Diebold et al., 2013; Ducros et al., 2013). For example, ultrasound encoding (Wang et al., 2012; Judkewitz et al., 2013), which frequencytags light emissions from a known location via a mechanical Doppler shift of the emitter (Mahan et al., 1998), provides a generic mechanism to sidestep problems of elastic optical scattering, although it requires distinguishing $\mathrm{MHz}$ frequency modulations in $\mathrm{THz}$ light waves (part per million frequency discrimination). Radio-frequency tagging of light emissions via a digitally synthesized optical approach is also an option and may be applicable to combatting the problem of emission scattering in deep-tissue, multi-point, multi-photon imaging (Diebold et al., 2013).

4.2.1.3. Speed of beam scanning. The speed of scanning microscopes is currently limited by beam repositioning times $(\sim 0.1 \mu \mathrm{s}$ for spinning disk (Flusberg et al., 2005; Kang et al., 2010; Mahalati et al., 2013), $3 \mu$ s for piezo-controlled linear scan mirrors, $\sim 10 \mu$ s for acousto-optic deflectors (Vučinić and Sejnowski, 2007), $\sim 8 \mathrm{kHz}$ line scans for resonant galvanometer mirrors). The $10 \mu$ s repositioning time for acousto-optic deflectors is set by the speed of sound in the deflector crystal, while scanning mirrors and spinning disks are limited by inertia. Note that $0.1 \mu \mathrm{s}$ repositioning time for current spinning-disk confocal techniques would require $10 \mathrm{~s}$ per frame for whole mouse brain imaging with a single scanned beam $\left(10^{-7}\right.$ s/site $\times$ $10^{8}$ sites/brain). There is therefore a need for a $10^{4}$ fold improvement in beam repositioning time and/or beam parallelization in order to achieve $1 \mathrm{kHz}$ imaging frame rates for whole mouse brains.

One strategy to implement parallelization would exploit (yet to be developed) fast, high-resolution phase modulator arrays to arbitrarily re-shape coherent optical wavefronts for multisite holographic multi-photon excitation in 3D (Papagiakoumou et al., 2010; Vaziri and Emiliani, 2012; Alivisatos et al., 2013). With fast phase modulation (e.g., $\sim 1 \mathrm{GHz}$ ), beating each excitation spot at a different frequency could allow a single detector to probe multiple sites in parallel, despite arbitrarily-large scattering of the outgoing light (Alivisatos et al., 2013). Emerging optical techniques may provide alternative means to implement similar strategies (Diebold et al., 2013). Temporal multiplexing of excitation pulses at distinct locations (e.g., via few-nanosecond beam delays) also allows parallelization of the excitation beam while combatting scattering ambiguity of the emitted light (Cheng et al., 2011). Furthermore, temporal focusing techniques in two-photon microscopy (depth-dependent pulse duration) can excite an entire plane or line within the sample (Oron et al., 2005; Tal et al., 2005; Sela et al., 2013; Packer et al., 2013), as well as arbitrary patterns of points (Papagiakoumou et al., 2010), potentially allowing 
fast axial scanning (somewhat analog to light-sheet techniques used with transparent samples). This method intrinsically corrects for scattering of the excitation light (Papagiakoumou et al., 2013), although not of the emission light. Like other multiphoton techniques, however, all these methods remain highly dissipative, as discussed below.

Fluorescence lifetimes in the $0.1-1$ ns range (Striker et al., 1999) ultimately constrain the design of scanning fluorescence microscopies. A delay of $0.1 \mathrm{~ns}$ per mouse neuron per frame corresponds to only $100 \mathrm{~Hz}$ frame rate without parallelization, implying that parallelization into at least 10 to 100 beams is essential. The fluorescence lifetime also limits the achievable modulation frequencies in beat-frequency-multiplexed parallelization strategies (Diebold et al., 2013), bit lengths in encoded strategies (Ducros et al., 2013), and temporal offsets in temporallymultiplexed strategies (Cheng et al., 2011), suggesting that parallelization of detectors may be necessary in a strongly scattering environment. Depending on the degree of parallelization, which constrains the achievable dwell times given a fixed frame rate, photon counts may also become a limiting factor for high-speed scanning in some approaches.

4.2.1.4. Diffraction. Using the small angle approximation, the diffraction-limited angular resolution of an aperture is $\theta \approx \frac{\Delta x}{y} \approx$ $\frac{\lambda}{D}$, where $\Delta x$ is the spacing which must be resolved, $y$ is the imaging depth, $\lambda$ is the wavelength, and $D$ is the aperture diameter. Thus distinguishing neurons which are $10 \mu \mathrm{m}$ apart and at a depth of $10 \mathrm{~mm}$ requires a lens aperture $D$ of $>1 \mathrm{~mm}$ when $\lambda \approx 1 \mu \mathrm{m}$. Diffraction therefore does not appear to be a limiting factor for cellular resolution imaging, except in the context of microscale apertures that might find use in embedded optics approaches.

\subsubsection{Energy dissipation}

Light that does not leave the brain is ultimately dissipated as heat. The total light power requirements for optical measurement of neuronal activity using fluorescent indicators depend on factors including fluorophore quantum efficiency, absorption crosssection, activity-dependent change in fluorescence, background fluorescence, labeling density, activation kinetics, detector noise, scattering and absorption lengths, and others. Unfortunately, many of these variables are unknown or highly dependent on particular experimental parameters.

A statistical analysis of photon count requirements for spike detection (in the context of calcium imaging) can be found in (Wilt et al., 2013), which derived a relationship between the number of background photon counts $\left(N_{\mathrm{bg}}\right)$ and the number of signal photon counts required for high fidelity spike detection given photon shot noise. This scales roughly as $N_{\text {signal }}>3 \sqrt{2 N_{\mathrm{bg}}}$, even at low absolute photon count rates. While this analysis governs the number of detected photons, the number of emitted photons will be higher due to losses. In one example using two-photon excitation, $5 \%$ of the emitted photons were captured by the photodetector (Kim et al., 1999). One implication of photon shot noise is that faster-responding indicators (e.g., voltage indicators which respond in near-real-time to the membrane potential) must be brighter.
4.2.2.1. Multi-photon excitation. Multi-photon experiments rely on short laser pulses with high peak light intensities at a focused excitation spot to excite non-linear transitions (Kim et al., 1999). This imposes an experimentally relevant physical limit: at least one excitation pulse of sufficient intensity per neuron per frame is required in order to excite multi-photon fluorescence during each frame. Assuming $1 \mathrm{kHz}$ frame rate and $0.1 \mathrm{~nJ}$ pulses (Cheng et al., 2011), delivering only one pulse per neuron per frame would dissipate roughly $\left(10^{8} \times 1 \mathrm{kHz} \times 0.1 \mathrm{~nJ}\right) 10 \mathrm{~W}$ in the mouse brain, which is clearly prohibitive. This is a lower bound because, in general, more than one excitation pulse per neuron per frame may be required to excite detectable fluorescence [e.g., one reference reported 12 pulses per spot (Kim et al., 1999)]. For three-photon excitation, the situation will be even worse as higher peak light intensities are required to excite three-photon fluorescence.

Could the single-pulse energy be reduced while maintaining efficient two-photon excitation? The number of two-photon (2P) transitions excited per fluorophore per pulse is $n_{a}=F^{2} C / t$, where $F$ is the number of photons per pulse per area in units of photon $/ \mathrm{cm}^{2}, C$ is the two-photon cross-section in units of $\mathrm{cm}^{4} \mathrm{~s} /$ photon, and $t$ is the pulse duration in seconds. This can be approximated as

$$
n_{a}=\left(\frac{\frac{E}{h c / \lambda}}{\left(\frac{\lambda}{2(\mathrm{NA})}\right)^{2}}\right)^{2} \frac{C}{t}=\left(\frac{4 E(\mathrm{NA})^{2}}{h c \lambda}\right)^{2} \frac{C}{t}
$$

where NA is the numerical aperture of the focusing optics, $E$ is the pulse energy and $\lambda$ is the stimulation wavelength. For a $2 \mathrm{P}$ experiment with $100 \mathrm{fs}, 0.1 \mathrm{~nJ}$ pulses, assuming a $2 \mathrm{P}$ cross section (Masters, 2006; Drobizhev et al., 2011) of $10^{-48} \mathrm{~cm}^{4} \mathrm{~s} /$ photon (i.e., 100 Goeppert-Mayer units Goeppert-Mayer, 1931, comparable to that of DsRed2 Drobizhev et al., 2011), $\lambda=900 \mathrm{~nm}$ and $\mathrm{NA}=1.0, n_{a} \approx \frac{1}{2}$. Thus, a few pulses are likely necessary and sufficient to excite $2 \mathrm{P}$ fluorescence by each fluorophore within the focal spot. With a $2 \mathrm{P}$ cross section above $10^{-47} \mathrm{~cm}^{4} \mathrm{~s}$ /photon (1000 Goeppert-Mayer units, higher than that of any fluorescent protein that we are aware of Drobizhev et al., 2011), one could reduce the pulse energy by an order of magnitude (and hence $n_{a}$ by two orders of magnitude) while maintaining $n_{a}>\frac{1}{20}$, i.e., one in 20 fluorophores excited by each pulse. Reducing the pulse energy much further might lead to unacceptably low excitation levels. Alternatively, shorter pulse durations could increase the light intensity, and hence $2 \mathrm{P}$ excitation probability, at fixed pulse energy.

Quantum dots can have $2 \mathrm{P}$ cross sections much higher than those of fluorescent proteins: water-soluble cadmium selenidezinc sulfide quantum dots have been reported with $2 \mathrm{P}$ cross sections of 47000 Goeppert-Mayer units and are compatible with in vivo imaging (Larson et al., 2003). These would allow excitation efficiencies of $n_{a}>\frac{1}{20}$ at $\mathrm{pJ}$ pulse energies, bringing whole-brain $2 \mathrm{P}$ imaging into the $\sim 100 \mathrm{~mW}$ range. Thus, the use of quantum dots or other ultra-bright multi-photon indicators could be decisive for supporting the energetic feasibility of multi-photon methods at whole brain scale; there are also plausible strategies for 
coupling quantum dot fluorescence to neuronal voltage (Marshall and Schnitzer, 2013). However, some quantum dots have long fluorescence lifetimes (Dahan et al., 2001), which may constrain scan speed.

For comparison to current practice, in a typical multi-photon experiment on mice, $\sim 50 \mathrm{~mW}$ of time-averaged laser power at the sample was used with a dwell time of $\sim 3 \mu$ s (Wilson et al., 2007), corresponding to $\sim 150 \mathrm{~nJ}$ energy dissipation per spot per frame. This dwell time would allow imaging only $\sim 300$ neurons at millisecond resolution with a single scanned excitation beam. The average excitation power here is likely already close both to whole-brain thermal dissipation limits, and to photo-damage limits for pulsed two-photon excitation (König et al., 1997; Hopt and Neher, 2001).

\subsubsection{Bioluminescence}

To work around the requirement for large amounts of excitation light, bioluminescent rather than fluorescent activity indicators could be used (Martin et al., 2007; Martin, 2008; Naumann et al., 2010). Consider a hypothetical activity-dependent bioluminescent indicator emitting at $\sim 1700 \mathrm{~nm}$ (IR), in order to evade light scattering. As a crude estimate, assuming that 100 photons must be collected by the detector per neuron per $1 \mathrm{~ms}$ frame, and $1 \%$ light collection efficiency by the detector relative to the emitted photons, $\sim 100 \mathrm{~mW}$ of bioluminescent photons emissions are required for the entire mouse brain (using $E_{\text {photon }}=$ $h c / \lambda)$. This would be feasible from the perspective of heat dissipation. By contrast, in a 1-photon fluorescent scenario, if 100 excitation photons must be delivered into the brain to generate a single fluorescent emission photon, the power requirement becomes $10 \mathrm{~mW}$, which is on the threshold of the steady-state heat dissipation limit. Therefore, bioluminescent indicators could potentially circumvent problems of heat dissipation even in the 1-photon case.

The widely used bioluminescent protein firefly luciferase is $\sim 80 \%$ efficient in converting ATP hydrolysis coupled with luciferin oxidation into photon production, yielding $\sim 0.8$ photons per ATP-luciferin pair consumed (Seliger and McElroy, 1960 ), and has $\sim 90 \%$ energetic efficiency in converting free energy to light production. Heat dissipation associated with the luciferase biochemistry itself is therefore not a significant overhead relative to the $100 \mathrm{~mW}$ of emitted photons calculated above. In the same scenario, however, each neuron would consume $\sim 6 \times$ $10^{8}$ additional ATP molecules per minute in order to power the bioluminescence, which is within the limits of cellular aerobic respiration rates $\left(\sim 1 \mathrm{fmol} \mathrm{O}_{2}\right.$ per minute per cell Molter et al., 2009, with $\sim 30$ ATP per $6 \mathrm{O}_{2}$, hence $3 \times 10^{9}$ molecules ATP synthesized per minute from ADP via glucose oxidation), but not by a large margin. Transient increases in metabolic rate are possible: energy dissipation more than doubles in the mouse during high physical activity (Speakman, 2013). Therefore, whole-brain activitydependent bioluminescence, at speeds high enough to achieve millisecond frame rates, may be metabolically taxing for the cell but is nevertheless plausible as a light generation strategy. Note that we have not treated the energy required to bio-synthesize the luciferin compound, which may create additional overhead (though conceivably luciferin could be provided exogenously).

\subsubsection{Conclusions and future directions}

Scattering of visible light in the brain creates a problem of signal-separation from deep-brain neurons. Multi-photon techniques, which scan an infrared excitation beam, can work around this scattering problem. However, current multi-photon techniques using fluorescent protein indicators, when applied at whole brain scale, would dissipate too much power to avoid thermal damage to brain tissue. Systems [such as plasmonic nano-antennas (Blanchard et al., 2011) or subwavelength metallic gratings (Harats et al., 2011)] that could locally excite multiphoton fluorescence without the need for high-energy laser pulses could conceivably ameliorate this issue. Importantly, quantum dots show promise as ultra-bright multi-photon indicators, if they can be targeted to neurons and optimized in terms of fluorescence lifetime. New methods besides multi-photon techniques could also work around the scattering of visible light in the brain. For example, fluorophores or bio-luminescent proteins could be developed which operate at infrared wavelengths. A compelling example from nature is the black dragonfish, which generates far red light $(\sim 705 \mathrm{~nm})$ via a multi-step bioluminescent process (using this light to see in deep ocean waters) (Widder et al., 1984; Campbell and Herring, 1987). A large set of activity indicators with distinguishable colors, generated through a combinatorial genetic recombination mechanism such as BrainBow (Livet et al., 2007), could also improve signal separation. Targeting, via protein tags, of activity indicators to specific locations-such as the axon, soma, soma and proximal dendrites, distal dendrites, pre-synaptic terminals, post-synaptic terminals, or intact synapses-could also aid in signal discrimination (El-Husseini et al., 2001; Jacobs et al., 2003; Boeckers et al., 2005; Arnold, 2007; Feinberg et al., 2008; Vacher et al., 2008; Corrêa et al., 2009; Yamagata and Sanes, 2012). In addition, implanted optical devices, which place emitters and detectors within a few scattering lengths of the neurons being probed, could potentially obviate the negative effects of scattering and allow visible-wavelength indicators to be used without a need for multi-photon excitation. In principle, excitation and detection do not need to make use of the same modality. For example, photoacoustic microscopy (Filonov et al., 2012) uses pulsed laser excitation to drive ultrasonic emission, leading to optical absorption contrast. Such asymmetric techniques impose fundamentally different requirements from pure-optical techniques relative to fluorophore properties, required light intensities and other parameters.

\subsection{EMBEDDED ACTIVE ELECTRONICS}

The preceding sections have assumed that electrical or optical signals from the recorded neurons are shuttled out of the brain before digitization and storage, but it is also conceivable to develop embedded electronic systems that locally digitize and then store or transmit (e.g., wirelessly) measurements of the activities of nearby neurons. This could allow for shorter wires in electrical recording approaches, and for shorter light path lengths in optical recording approaches, as well as for more facile (e.g., non-surgical) delivery mechanisms for the recording hardware.

Integrated circuits have shrunk to a remarkable degree: in about 3 years, following the Moore's law trajectory, it will likely be possible to fit the equivalent of Intel's original 4004 
micro-processor in a $10 \times 10 \mu \mathrm{m}$ chip area. Functional wirelessly powered radio-frequency identification (RFID) chips as small as $50 \mu \mathrm{m}$ in diameter have been developed (Usami et al., 2007) and tags with chip-integrated antennas function at the $400 \mu \mathrm{m}$ scale (Impinj, Inc.). Integrated neural sensors including analog front ends are also scaling to unprecedented form factors: a $250 \times 450 \mu \mathrm{m}$ wireless implant-including the antenna, but not including a $\sim 1 \mathrm{~mm}$ electrode shank used to separate signal from ground-draws only $2.5 \mu \mathrm{W}$ per recording channel (Biederman et al., 2013). The system operates at $\sim 1 \mathrm{~mm}$ range in air, powered by a transmitter generating $\sim 50 \mathrm{~mW}$ of transmitted power. Note that for a single such embedded recording device, the heat dissipation constraint is set not by the device's own dissipation (10 $\mu \mathrm{W}$ for four recording channels) but rather by the RF specific absorption rate limit associated with the $50 \mathrm{~mW}$ transmit power.

Possibilities may exist for non-surgical delivery of embedded electronics to the brain: remarkably, cells such as macrophages $(\sim 13 \mu \mathrm{m}$ in size $)$ can engulf structures up to at least $20 \mu \mathrm{m}$ in diameter (Cannon and Swanson, 1992) and have been studied as potential delivery vehicles for nano-particle drugs (Kadiu et al., 2011), suggesting that they might be used to deliver tiny microchips. T-cells and other immune cells can trans-migrate across the blood brain barrier (Engelhardt, 2006) and ghost cells (membranes purged of their contents) engineered to encapsulate synthetic cargo (Cinti et al., 2011) can fuse with neurons (Hikawa et al., 1989). It might even be possible to engineer such cell-based delivery vehicles to form electrical gap junctions (Spruston, 2001) with neurons or to act as local biochemical sensors (Nguyen et al., 2009).

The real-time transmission bandwidth requirements for neural recording could be significantly reduced if it is only desired to take a "snapshot" of neural activity patterns over a limited period of time, but this would require a large amount of local storage. For example, flash memory can store $>10$ Mbit of data in a device $100 \mu \mathrm{m}$ on a side: a 64 giga-byte microSD card with $1.5 \mathrm{~cm}^{2}$ area corresponds to 34 mega-bits per $(100 \mu \mathrm{m})^{2}$ area. Even denser forms of memory storage are under development and could perhaps be used in a one-time-write mode in the context of neural recording long before they become commercially viable for use as rewritable media in the electronics industry.

Here we consider the power dissipation associated with embedded electronic recording devices, as well as the constraints on possible methods to power them. In the next section, we describe how physics constrains the data transmission rates from such devices.

\subsubsection{Power Requirements for Recording}

Any embedded system needs to process data, in preparation for either local storage or wireless transmission. Physics defines hard limits on the required power consumption associated with data processing (neglecting the possibility of reversible logic architectures Bennett, 1973), arising from the entropy cost for erasing a bit of information (Landauer, 1961):

$$
E_{\text {Landauer }}=\ln (2) k_{\mathrm{B}} T \approx 3 \times 10^{-21} \mathrm{~J} / \mathrm{bit} \quad \text { (the Landauer limit) }
$$

Ambitious yet physically realistic values for beyond-CMOS logic lie in the tens of $k_{\mathrm{B}} T$ per bit processed (Yablonovitch, 2008).
Scaling $40 k_{\mathrm{B}} T /$ bit to record raw voltage waveforms at a minimal $1 \mathrm{kbit} / \mathrm{s} /$ neuron (e.g., $1 \mathrm{kHz}$ sampling rate, 1 bit processed per neuron per sample), the total power consumption for whole mouse brain recording could in principle be as low as $\sim 16 \mathrm{nW}$. While this leaves $>10^{6}$-fold more room (energetically) for increased data processing (more required bit flips per second), or energetic inefficiency of the switching device (greater dissipation per bit), realistic devices in the near-term may in fact require this much overhead, if not more. This necessitates a more detailed consideration of limiting factors for today's microelectronic devices.

In the context of electrical recording, the first step that must be performed by an embedded neural recording device is digitization of the voltage waveform. Until $\mathrm{mV}$-scale switching devices are developed (see discussion below), it is necessary to amplify the $\sim 10-100 \mu \mathrm{V}$ spike potential in order to drive digital switching events in downstream gates. During this sub-threshold amplification step, a CMOS (or BJT) device will dissipate static power (associated with a bias current). Importantly, in order to decrease the input-referred voltage noise of this amplification process, it is necessary to increase the bias current and hence the static power dissipation. For a simple differential transistor amplifier, the minimal bias current scales as

$$
I_{\mathrm{d}}=\frac{\pi}{2} \frac{4 k_{\mathrm{B}} T}{V_{\text {noise }}^{2}} \frac{k_{\mathrm{B}} T}{q} \mathrm{BW}
$$

where $V_{\text {noise }}$ is the input-referred voltage noise of the amplifier and $q$ is the electron charge. For an extracellular recording with $\mathrm{BW}=10 \mathrm{kHz}$ and $V_{\text {noise }}=10 \mu \mathrm{V}$, this implies a minimal bias current $I_{\mathrm{d}} \approx 60 \mathrm{nA}$ or a minimal static power of $\left(I_{\mathrm{d}} V_{\mathrm{dd}}\right) \approx$ $6 \times 10^{-8} \mathrm{~W}$ at $V_{\mathrm{dd}} \approx 1 \mathrm{~V}$ operating voltage. Assuming 10 neurons per recording channel, there are then 7.5 million recording channels for a mouse brain, which gives a power dissipation associated with signal amplification of $\sim 500 \mathrm{~mW}$. Note that realistic analog front ends (which are subject to $1 / f$ noise and require multiple gain stages) draw $6 \times-10 \times$ greater bias current, quantified by the noise efficiency factor (NEF) (Steyaert et al., 1987), to achieve the same input-referred noise levels.

Local on-chip digital computation also incurs an energy cost. Current CMOS digital circuits consume 5-6 orders of magnitude (Yablonovitch, 2008; Koomey et al., 2011; Tucker, 2011; Tucker and Hinton, 2011) more energy per switching event $(\sim 1 \mathrm{fJ} / \mathrm{bit}$ including charging of the wires Tucker and Hinton, 2011) compared to the Landauer limit (e.g., for a digital CMOS inverter, and ignoring the static power associated with the leakage current). This corresponds to a $\sim 1 \mathrm{fF}$ total load capacitance at $1 \mathrm{~V}$ operating voltage. For $100 \mathrm{GHz}$ switching rates $\left(10^{8}\right.$ neurons $\times$ $1 \mathrm{kHz}$ ) as above, this corresponds to $0.01-0.1 \mathrm{~mW}$. Realistic architectures, however, will incur overhead in the number of switching events required to store, compress and/or transmit neural signals, likely bringing the power consumption into an unacceptable range (e.g., 1000 bits processed per sample would be $100 \mathrm{~mW}$ here). To take a concrete example, commercial RFID tags consume $\sim 10 \mathrm{~mW}$ (Fraunhofer Institute for Photonic Microsystems, 2011). At a chip rate of $256 \mathrm{kbit} / \mathrm{s}$ (with a Miller encoding of 2), this yields $7.8 \times 10^{-11} \mathrm{~J} / \mathrm{bit}$, which is $\sim 10$ orders of magnitude 
higher than the Landauer limit. Applying current RFID technology to whole mouse brain recording at $1 \mathrm{kbit} / \mathrm{s} /$ neuron would thus draw $\sim 8 \mathrm{~W}$ of power. Therefore, at least $2-3$ orders of magnitude reduction in power consumption will be necessary in order to apply embedded electronics for whole-brain neural recording.

Until recently, the energy efficiency of digital computing has scaled on an exponential improvement curve (Koomey et al., 2011). This was a consequence of Moore's law and Dennard scaling, where both the capacitance of each transistor and its associated interconnect, as well as the operating voltages, were reducing with the device dimensions. Unfortunately, issues related to device variability and the $3 \mathrm{D}$ structures needed to maintain the on-to-off current ratio have largely stopped the reduction in effective capacitance per device; current devices are stuck at $\sim 100-200 \mathrm{aF}$ for a minimum sized transistor. Furthermore, the exponential increase in leakage current that comes along with the scaling of the threshold voltage in this scenario has precluded substantial further decreases in voltage at a given performance level. Indeed, for the past several technology generations (since about 2005), CMOS devices have operated at a supply voltage of $\sim 1 \mathrm{~V}$.

While neural signal processing does not demand very stringent transistor speeds and so reductions below $\sim 1 \mathrm{~V}$ are certainly feasible, a fundamental limitation in scaling the supply voltage still remains. Specifically, CMOS has a well-defined minimum-energy per bit and an associated minimum-energy operating voltage that is defined by the tradeoff between static (leakage) and dynamic (switching) energy: as the operating voltage is decreased, the capacitive switching energy decreases, but the ratio of currents in the on and off states, $I_{\text {off }} / I_{\text {on }}$, increases exponentially, increasing the energy associated with leakage (this effect is independent of the threshold voltage in the sub-threshold regime). For practical circuits, the supply voltage that leads to this minimum energy is on the order of $300-500 \mathrm{mV}$, and thus supply voltage scaling will at most provide $3 \times-10 \times$ improvement in energy over today's designs.

Thus, a paradigm shift in microelectronic hardware is needed to reduce power by several orders of magnitude if we are to approach the physical limits. Developing a switching device operating in the $\mathrm{mV}$ range, rather than the $1 \mathrm{~V}$ range of current transistors, would allow $(1 \mathrm{~V} / 1 \mathrm{mV})^{2}=10^{6}$ fold reduction in power consumption (Yablonovitch, 2008). Electronic circuits constructed using analog techniques (Sarpeshkar, 1998), which sometimes rely on bio-inspired computational architectures, show promise for reducing energy costs by up to five orders of magnitude (Sarpeshkar, 1998; Mandal and Sarpeshkar, 2007; Rapoport et al., 2009), depending on the nature of the computation and the required level of precision.

Figure 4 shows the power consumption per bit processed for several technology classes as well as the corresponding total power consumption required for whole brain readout, assuming a minimal whole-brain bit rate of $100 \mathrm{Gbit} / \mathrm{s}$.

\subsubsection{Powering embedded devices}

Embedded systems need power, which could be supplied via electromagnetic or acoustic energy transfer, or could be harvested from the local environment in the brain.

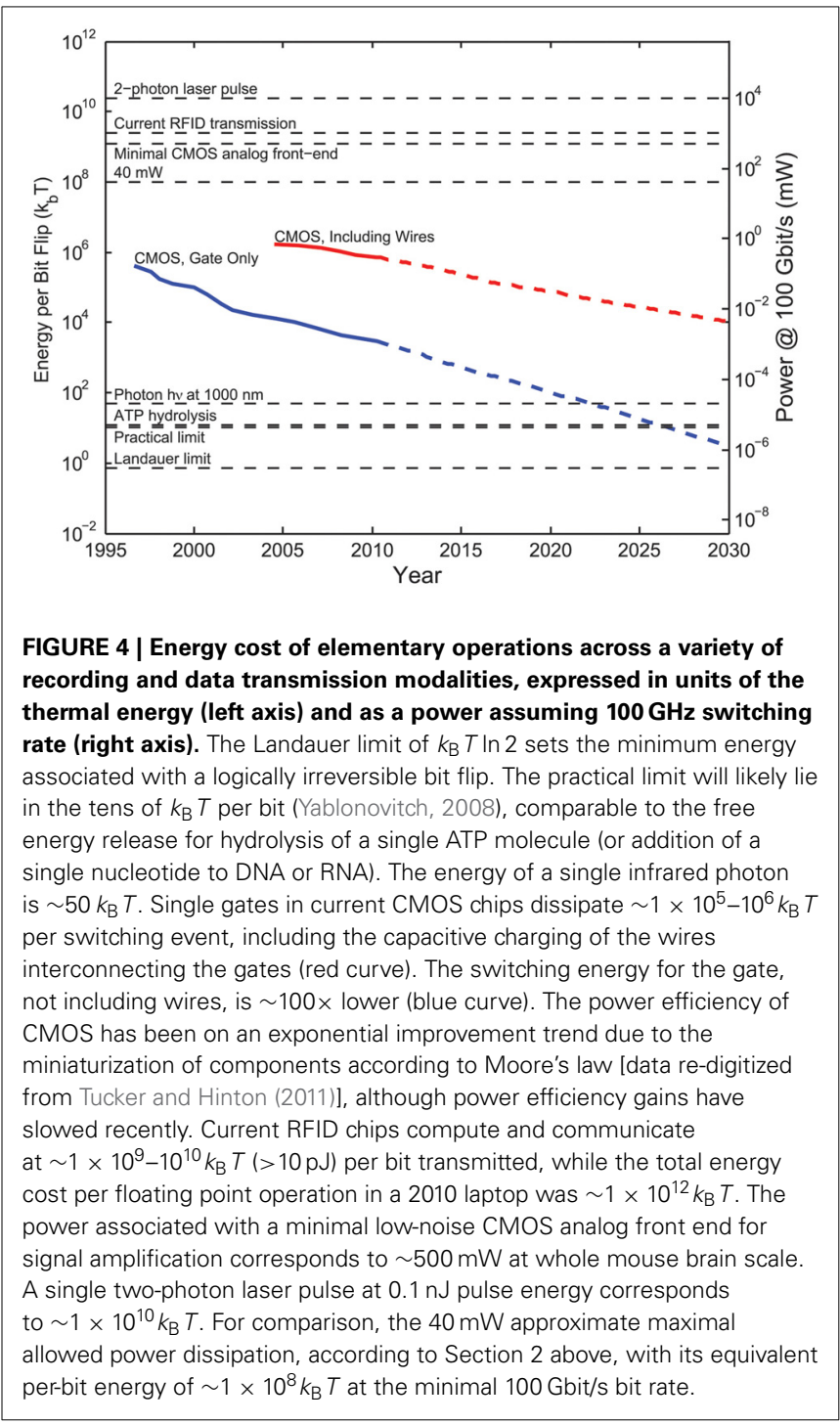

There are two key regimes for wireless electromagnetic power transfer: non-linear device rectification and photovoltaics. If the single-photon energy is sufficient to allow electrons to move from the valence to the conduction band-that is, band gap $<h v / q$, where $q$ is the electron charge, $h$ is Planck's constant, and $v$ is the frequency of the photon-a photovoltaic effect can occur. Otherwise, electromagnetic energy is converted to voltage by an antenna and non-linear device rectification may occur.

When photon energies are much lower than the band gap, power conversion is governed by the total RF power and by the impedances of the antenna and the rectifier, rather than by the individual photon energy. For a monochromatic RF source, there is no thermodynamic or quantum limit to the RF to DC conversion efficiency, other than the resistive losses and threshold voltages for a semiconductor process. For rectification, when the input voltage to the rectifier is much higher than a semiconductor process threshold, conversion efficiencies of $85 \%$ have been achieved (Sun and Chang, 2002). At low input voltages relative to 
the semiconductor process threshold, efficiencies as high as $25 \%$ and $2 \mu \mathrm{W}$ load have been achieved [see (Mandal and Sarpeshkar, 2007) for an analysis of power efficiency]. Ultimately, rectification improvements are dependent on the same improvements which will be needed for next-generation low-power computing: $\mathrm{mV}$ scale switching devices (promising research directions include tunnel FETs (Ionescu and Riel, 2011), electromechanical relays (Liu et al., 2012) and other options).

While efficient rectification is thus not a fundamental issue, capturing sufficient RF energy in the first place becomes increasingly challenging as microchips become smaller and more deeply embedded in tissue. Wireless electromagnetic power transfer imposes range constraints due to the loss in power density with distance. For directional power transfer, placing the receiver at the edge of the transmitter's near field (the Rayleigh distance $\frac{D^{2}}{4 \lambda}$ where $D$ is the transmitter aperture) has advantages in terms of energy capture efficiency (Ozeri and Shmilovitz, 2010), whereas for omni-directional antennas it is advantageous to place the receiver as close as possible to the transmitter. If embedded chips are oriented randomly with respect to the transmitter, the radiation patterns of their antennas cannot be highly directional, i.e., their gains $G_{r}$ (a measure of directionality) must be close to one. In the far field, this lack of directionality limits power capture by the antenna [due antenna reciprocity (Gershenfeld, 2000)]: the maximal power $P_{A}$ available to the chip is

$$
P_{A}=\frac{G_{r} P_{\mathrm{rad}} \lambda^{2}}{4 \pi}
$$

where $P_{\text {rad }}$ is the power density of radiation around the antenna, $\lambda$ is the wavelength and $G_{r} \approx 1$ for a non-directional antenna (Mandal and Sarpeshkar, 2007).

It may be possible to power devices with pure magnetic fields (which are highly penetrant) via near-field (non-radiative) inductive coupling, which is widely used in systems ranging from biomedical implants to electric toothbrushes, or conceivably by using magneto-electric materials (Fiebig, 2005; Priya et al., 2009; Kitagawa et al., 2010; Yue et al., 2012). For the case of simple inductive coupling, however, the tiny cross-sections of microdevices limit the amount of power which can be captured: a loop of $10 \mu \mathrm{m}$ diameter in an applied field of $1 \mathrm{~T}$ switching at $1000 \mathrm{~Hz}$ produces an induced electromotive force of only $0.1 \mu \mathrm{V}$. Assuming a copper loop $(\sim 17 \mathrm{n} \Omega \mathrm{m}$ resistivity) with $1 \times 1 \mu \mathrm{m}$ cross-section and $40 \mu \mathrm{m}$ length (around the outer edge of the chip) gives a power $\left(V^{2} / R\right)$ of only $\sim 15 \mathrm{fW}$ associated with the induced current. In general, the use of coupled high- $Q$ resonators can increase the range and efficiency of near-field electromagnetic power transfer by orders of magnitude (Karalis et al., 2008) compared to non-resonant inductive power transfer and may be particularly relevant for implanted devices (Ho et al., 2013). Unfortunately, at the $\sim 10 \mu \mathrm{m}$ length scale, the achievable onchip inductances and capacitances are severely limited, which restricts the operating range of any resonant device to high frequencies $\left(f_{\text {resonant }}=(2 \pi \sqrt{L C})^{-1}\right)$ which will be attenuated by tissue. Electromagnetic near-field power transfer though tissue to ultra-miniaturized microchips may thus be inefficient, again due to low capture efficiency of the applied fields by tiny device cross-sections.

Alternatively, if the photon energy is above the silicon band gap ( $\lambda<\frac{h c}{q V_{\text {th }}} \approx 3 \mu \mathrm{m}$ or less for silicon), the chip is essentially acting as a photovoltaic cell. There is no thermodynamic or quantum limit to the conversion efficiency of light to DC electrical power for monochromatic sources, other than resistive losses and dark currents in the material ( $86 \%$ in GaAs for example Bett et al., 2008). Again, however, capturing sufficient light becomes difficult for tiny devices. To supply $10 \mu \mathrm{W}$ (typical of current wirelesslypowered RFID chips) photovoltaically to a $10 \times 10 \mu \mathrm{m}$ (cell sized) chip at $34 \%$ photovoltaic efficiency requires a light intensity of $\sim 300 \mathrm{~kW} / \mathrm{m}^{2}$ at the chip, which is prohibitive. Furthermore, in the use of infrared light for photovoltaics, the penetration of the photons through tissue is decreased compared to radio frequencies.

Piezoelectric harvesting of ultrasound energy by micro-devices is a possibility (Seo et al., 2013). The efficiency of electrical harvesting of mechanical strain energy in piezoelectrics can be above $30 \%$ for materials with high electromechanical coupling coefficients (e.g., PZT) (Ahmad and Akdoan, 2008; Xu et al., 2012). The losses in the piezoelectric transduction process are well described by models such as the KLM model (Krimholtz et al., 1970; Castillo et al., 2003).

An alternative to wireless energy transmission is the local harvesting of biochemical energy carriers. Implanted neural recording devices could conceivably be powered by free glucose, the main energy source used by the brain itself. The theoretical maximum thermodynamic efficiency for a fuel cell in aqueous solution is equal to that of the hydrogen fuel cell: $\Delta G^{0} / \Delta H^{0}=83 \%$ at $25^{\circ} \mathrm{C}$. Furthermore, if glucose is only oxidized to gluconic acid, the Coulombic (electron extraction) efficiency is at most $8.33 \%$ (Rapoport et al., 2012), which bounds the thermodynamic efficiency. The blood glucose concentration in rats has been measured at $\sim 7.6 \mathrm{mM}$, with an extracellular glucose concentration in the brain of $\sim 2.4 \mathrm{mM}$ (Silver and Erecińska, 1994). A hypothetical highly miniaturized neural recorder with a device area of $25 \times 25 \mu \mathrm{m}$ and efficiency of $80 \%$, processing a blood flow rate of $\sim 1 \mathrm{~mm} / \mathrm{s}$ (Ivanov et al., 1981) could extract $(80 \%)(7.6 \mathrm{mM})(25 \mu \mathrm{m})^{2}(1 \mathrm{~mm} / \mathrm{s})(2880 \mathrm{~kJ} / \mathrm{mol}) \approx$ $11 \mu \mathrm{W}$, which is sufficient for low-power device such as RFID chips (Cho et al., 2005). Unfortunately, current nonmicrobial glucose fuel cells obtain only $\sim 180 \mu \mathrm{W} / \mathrm{cm}^{2}$ peak power and $\sim 3.4 \mu \mathrm{W} / \mathrm{cm}^{2}$ steady state power (Rapoport et al., 2012). Thus there is a need for $10^{4}$ - and $10^{6}$-fold improvements in peak and steady state power densities, respectively, for non-microbial glucose fuel cells to power brain-embedded electronics of the complexity of today's RFID chips (or for the corresponding decrease in power requirements, as emphasized above).

\subsubsection{Conclusions and future directions}

The power consumption of today's microelectronic devices is more than six orders of magnitude higher than the physical limit for irreversible computing, and 2-3 orders of magnitude higher than would be permissible for use in whole brain millisecond resolution activity mapping, even under favorable 
assumptions on the required switching rates and neglecting both the power associated with noise rejection in the analog front end and the CMOS leakage current. Thus, the first priority is to reduce the power consumption associated with embedded electronics. In principle, methods such as infrared light photovoltaics, RF harvesting via diode rectification, or glucose fuel cells, could supply power to embedded neural recorders, but again, significant improvements in the power efficiency of electronics are necessary to enable this. Other potential energy harvesting strategies include materials/enzymes harnessing local biological gradients such as in voltage, osmolarity, or temperature. An analysis of the energy transduction potential of each of these systems is beyond the scope of this discussion. Fortunately, with many orders of magnitude potential for improvement before physical limits are reached, we may expect that embedded nano-electronic devices will emerge as an energetically viable neural interfacing option at some point in the future.

\subsection{EMBEDDED DEVICES: INFORMATION THEORY}

Most recording methods envisioned thus far rely on the real-time transmission of neural activity data out of the brain. Physics and information theory impose fundamental limits on this process, including a minimum power consumption required to transmit data through a medium. The most basic of these results hold irrespective of whether the data transmission is wired or wireless, and regardless of the particular physical medium (optical, electrical, acoustic) used as the information carrier.

A communication "channel" is a set of transmitters and receivers that share access to a single physical medium with fixed bandwidth. The bandwidth is the range of frequencies present in the time-varying signals used to transmit information. In wireless communications, information is transmitted by modulating a carrier wave. To allow modulation, the frequency of the carrier wave must be higher than the bandwidth: for example, a $400 \mathrm{THz}$ visible light wave may be modulated at a $100 \mathrm{GHz}$ rate. The physical medium underlying a channel could be a wire (with a bandwidth set by its capacitive RC time constant), an optical fiber, free space electromagnetic waves over a certain frequency range, or other media.

As a concrete example, consider a police department with 100 officers, each possessing a hand-held radio. The radios transmit vocalizations by modulating an $80 \mathrm{MHz}$ carrier wave at $\sim 10 \mathrm{kHz}$. This constitutes a single shared communications channel with $10 \mathrm{kHz}$ bandwidth. Simultaneously, the fire department may communicate via a separate channel, also with a bandwidth of $\sim 10 \mathrm{kHz}$, by modulating a $90 \mathrm{MHz}$ carrier wave. The channels are separate because modulation introduced into one does not affect the other. If the neighboring town's police department makes the mistake of also operating at $80 \mathrm{MHz}$ carrier frequency, then they share a channel and conflicts will arise.

\subsubsection{Power requirements for single-channel data transmission}

We first treat the case in which there is a single channel for transmitting data out of the brain. As discussed above in the context of electrical spike sorting, the Shannon Capacity Theorem
(Cover and Thomas, 2006) sets the maximal bit rate for a channel (assuming additive white Gaussian noise) to

$$
R_{\max }=\mathrm{BW} \log _{2}(1+\mathrm{SNR})
$$

where BW is the channel bandwidth and SNR is the signal-tonoise ratio. If there is only thermal noise the $\mathrm{SNR}=P /\left(N_{0} \mathrm{BW}\right)$, where $N_{0}$ is the thermal noise power spectral density of $k_{\mathrm{B}} T \mathrm{~W} / \mathrm{Hz}$ and $P=(\mathrm{PL}) P_{0}$ is the power of the transmitted signals $P_{0}$, weakened by path loss PL. Therefore the transmitted power $P_{0}$ is lower-bounded:

$$
P_{0}>k_{\mathrm{B}} T \mathrm{BW} \frac{2^{R_{\max } / \mathrm{BW}}-1}{\mathrm{PL}}
$$

as shown in Figure 5 (bottom). In a minimal model of a transmitter-receiver system, there thus exists a tradeoff between the required signal power and the bandwidth of the carrier radiation, due to the thermal noise floor, even in the absence of path loss $(\mathrm{PL}=1)$.

Path loss weakens the proportion of the power that can reach the detector. Using the above equation, we can calculate, as a function of bandwidth, the power necessary to transmit a target whole-brain bit rate of $100 \mathrm{Gbit} / \mathrm{s}$ through a medium with path loss dependent on the carrier wavelength, as shown in Figure 5 (top).

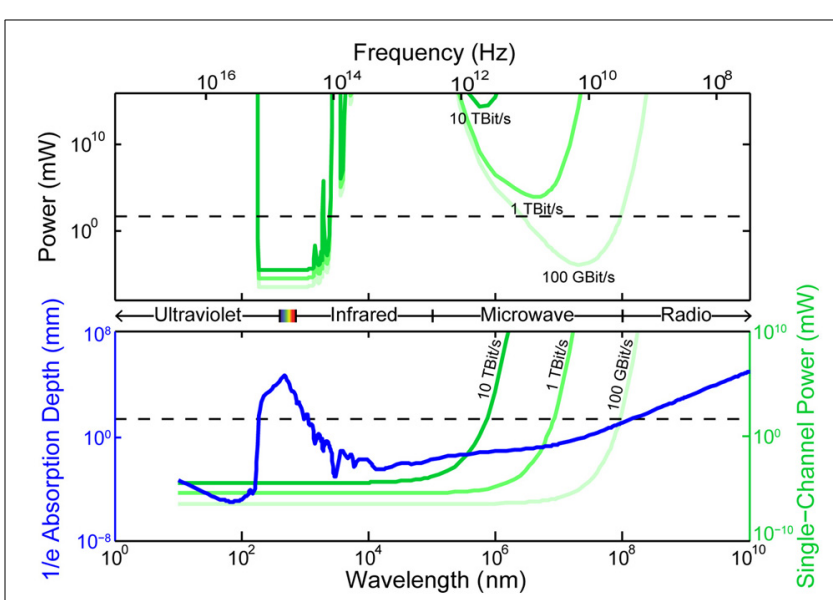

FIGURE 5 | Power requirements imposed by information theory on data transmission through a single (additive white Gaussian noise) channel with carrier frequency $v$ (an upper bound on the bandwidth), given thermal noise and path loss. Bottom: absorption length of water as a function of frequency (blue), minimal power to transmit data at 100 , 1000 , and $10,000 \mathrm{Gbit} / \mathrm{s}$ (green) as a function of frequency, assuming thermal noise but no path loss. Top: minimal power to transmit data at 100 1000 and $10,000 \mathrm{Gbit} / \mathrm{s}$ as a function of frequency, assuming thermal noise and a path loss corresponding to the attenuation by water absorption over a distance of $2 \mathrm{~mm}$. While formulated for a single channel, at certain wavelengths (e.g., RF) these factors also constrain multiplexed data transmissions between many transmitters and many receivers, depending on capacity of the system for spatial multiplexing. Horizontal dashed lines: $40 \mathrm{~mW}$, the approximate maximal whole-brain power dissipation in steady state. 
For RF wavelengths, the radiation penetrates deeply but the achievable data rates are low without excessive power consumption, due to the limited bandwidth. For wavelengths intermediate between RF and infrared, the penetration depth is low and power must be expended to combat these losses, despite the high carrier bandwidth. Only in the infrared and visible ranges do the tradeoffs between power, bandwidth and penetration depth allow transmission of $>100 \mathrm{Gbit} / \mathrm{s}$ out of the brain through a single channel without unacceptable power consumption.

The analysis above has ignored the effects of noise sources other than thermal noise, but many additional noise sources will increase the amount of power needed to transmit data, via a decrease in the SNR at fixed input power. For optical transmission in the brain, the noise is dominated by time-correlated "speckle noise" below $200 \mathrm{kHz}$, which arises mostly from local blood flow (Carp et al., 2011). This correlated noise, which cannot be filtered by simple averaging, could be avoided by modulating optical signals at frequencies above $200 \mathrm{kHz}$.

\subsubsection{Spatially multiplexed data transmission}

As discussed above, transmitting information through a single channel imposes direct limits on bit rate, carrier frequency and input power. However, it is conceivable to divide the data transmission burden over many independent channels, i.e., over many pairs of transmitters and receivers, each operating at lower bandwidth (e.g., at radio frequencies). Indeed, this would be optimal in a scenario where many embedded devices measure and then transmit the activities of nearby neurons. As a concrete example of such "spatial multiplexing," an effective capacity of $1 \mathrm{Tbit} / \mathrm{s}$ could conceivably be obtained by splitting the data over 1000 transmitter-receiver pairs each operating at $1 \mathrm{Gbit} / \mathrm{s}$, with the transmitters arranged in a $10 \times 10 \times 10$ grid. Importantly, in order to exceed the above limits for single-channel data transmission, it must be possible for these transmitter receiver pairs to share the same bandwidth and operate simultaneously without conflicts, for example by modulating distinguishable carrier waves or by transferring data over separate wires. The conditions under which this may occur, however, can be counter-intuitive. For example, for antennas to operate independently, they must be spaced apart from one another by roughly a wavelength. For $10 \mathrm{GHz}$ microwaves, the wavelength is $\sim 3 \mathrm{~cm}$, so no more than a handful of microwave transmitters (e.g., operating at frequencies in the $100 \mathrm{GHz}-1 \mathrm{THz}$ range) can co-occupy the mouse brain while operating independently.

Even with many non-independent transmitters co-occupying the brain and operating simultaneously over the same frequency spectrum, it may be possible under some conditions to "factor out" the effects of the coupling and allow an increase in channel capacity relative the single-channel result. To treat such scenarios, a generalization to Shannon's capacity theorem to multi-inputmulti-output (MIMO) channels has shown that the maximal total data rate is

$$
R_{\max }=\mathrm{BW} \cdot \log _{2}\left|\mathbf{I}+(\mathrm{SNR}) \mathbf{H H}^{*}\right|
$$

where $\mathbf{I}$ is the identity matrix, $|\cdot|$ denotes the matrix determinant, $\mathbf{H}$ is the $(M \times N$ for $N$ transmitters and $M$ receivers $)$ channel matrix giving the coupling between the vector of transmitted signals and the vector of received signals and $\mathbf{H}^{*}$ denotes the matrix adjoint of $\mathbf{H}$ (Tulino and Verdú, 2004). The vector of received signals is then $\mathbf{y}=\mathbf{H x}+\mathbf{n}$ where $\mathbf{x}$ is the vector of transmitted signals and $\mathbf{n}$ is a noise vector. Any matrix can be written as $\mathbf{H}=\mathbf{U} \mathbf{\Sigma} \mathbf{V}^{*}$ where $\mathbf{U}$ and $\mathbf{V}$ are unitary matrices, and $\boldsymbol{\Sigma}$ is a diagonal matrix whose elements are the singular values $\lambda_{i}$. One can re-write the above equation as

$$
R_{\max }=\mathrm{BW} \cdot \sum_{i=1}^{\min (M, N)} \log _{2}\left(1+\mathrm{SNR} \cdot \lambda_{i}^{2}\right)
$$

If the matrix $\mathbf{H}$ is of full rank, then the capacity for the multichannel system can increase over the single-input-single-output (SISO) result by $\min (M, N)$ times (Shiu et al., 2000). Note that the rank of the matrix corresponds to the number of non-zero singular values, so an analysis of the singular values of channel matrices can inform us about the multiplexing capacity of the channel. Furthermore, this multiplexing capacity can in principle be achieved even when the transmitters are not in communication with each other, which could potentially be important for scenarios involving many brain embedded transmitters (Spencer et al., 2004).

Transmission through a medium with negligible scattering is the simplest situation to analyze. In this case, evaluating the matrix $\mathbf{H}$ requires knowledge of the transmitter-transmitter, transmitter- receiver, and receiver-receiver distances, as well as the orientations and radiation patterns of the antennas (e.g., high gain antennas will have a highly directional radiation pattern). Depending on these factors, the beam from each transmitter will spread to impinge upon multiple receivers and the effective number of spatially independent beams will be reduced. With transmitter-transmitter and receiver-receiver distances larger than the wavelength, and highly directional antennas with appropriately chosen orientations, it is possible to increase the channel capacity linearly with $\min (M, N)$.

Random scattering, in a coherent disordered medium where the mean free-path $l$ is much larger than the wavelength $\lambda$ and much smaller than the size of the disordered medium, is another condition where the matrix $\mathbf{H}$ is a random scattering matrix of full rank (Moustakas et al., 2000; Popoff et al., 2010). Intuitively, for the case of two transmitters and two receivers separated by a disordered medium larger than the mean free path: if transmitter 1 is at least a mean-free path from transmitter 2 [or potentially as close as a few wavelengths (Berkovits, 1991)], the path from transmitter 1 to receiver 1 and the path from transmitter 2 to receiver 2 would be uncorrelated with respect to one another (in terms of physical path, phase, amplitude fluctuations, and other properties). The rank of the matrix $\mathbf{H}$ would then be 2 . Devising a code on the transmitter such that the receivers can distinguish between these two uncorrelated streams results in a doubling of the capacity, rather than simply averaging the noise floor, which would provide only a logarithmic capacity gain due to the increased SNR. 
Thus, contrary to intuition, a high degree of random scattering can potentially be useful for data transmission, by enabling spatial multiplexing of channels. This idea has been demonstrated experimentally in the context of ultrasound transmissions (Derode et al., 2003). Biological tissue in the infrared range is well described as such a random scattering medium (e.g., mean free path $\sim 200 \mu \mathrm{m}$ at $\sim 800 \mathrm{~nm}$ in vivo). Therefore infrared light could be used for spatially multiplexed data transmission out of the brain. At wavelengths $\lambda$ comparable to critical brain dimensions in the mouse, however, an insufficient number of scattering events will occur to create multiple independent pathways for $N$ transmitters. Mathematically, the matrix $\mathbf{H}$ will have one highly dominant singular value and a number of much smaller remaining terms, such that the signals appearing at a receiver from two separate transmitters will be highly linearly dependent, differing by only a small phase angle. Therefore, there will be no capacity gain from multiple transmitters, and distinct transmitters will effectively share a single channel (reducing to the SISO result).

Little is known about the biological interaction with electromagnetic fields at wavelengths much shorter than the critical brain dimensions but beyond the infrared, approximately $100 \mathrm{GHz}(\sim 3 \mathrm{~mm})$ to $100 \mathrm{THz}(\sim 3 \mu \mathrm{m})$ in the mouse. If multiple scattering occurs and the absorption is low, this may also be a regime conducive to MIMO communications (Bakopoulos et al., 2009). Efficiently generating and processing radiation in this regime by embedded devices is an outstanding problem, however. The so-called "THz-gap" (Tonouchi, 2007) exists because (moving toward higher frequencies starting from DC electronics), parasitic capacitances and passive losses limit the maximum frequency at which a field-effect transistor (FET) may oscillate and on the other hand (moving downward in frequency starting from optics), the band-gaps of opto-electronic devices limit the minimum frequency at which quantum transitions occur. Thus there is no high-power, low-cost, portable, room temperature $\mathrm{THz}$ source available. Advances in $\mathrm{THz}$ light generation, e.g. through the use of tunneling transistors, could be enabling.

\subsubsection{Ultrasound as a data transmission modality}

An important caveat to these conclusions on wireless data transfer occurs if we consider the use of ultrasound rather than electromagnetic radiation. Because the speed of sound is dramatically slower than that of light, the wavelength of $10 \mathrm{MHz}$ ultrasound is only $\sim 150 \mu \mathrm{m}$ (approximating the speed of sound in brain as the speed of sound in water, $\sim 1500 \mathrm{~m} / \mathrm{s}$ ). Thus, many $10 \mathrm{MHz}$ ultrasound transmitters/receiver could be placed inside a mouse brain while maintaining their spatial separation above the wavelength, and a linear scaling of the MIMO channel capacity with the number of devices is likely possible in this regime, assuming that appropriate antenna gains and orientations can be achieved inside brain tissue. Beam orientation could present a challenge if micro-devices are oriented randomly after implantation. With an attenuation of $0.5 \mathrm{~dB} / \mathrm{cm} / \mathrm{MHz}$ (Hoskins, 2010), the attenuation at $10 \mathrm{MHz}$ is only $5 \mathrm{~dB} / \mathrm{cm}$. Thus ultrasound-based transmission of power and data from embedded recording devices may be viable (Seo et al., 2013).

In contrast, direct imaging of neural activity by ultrasound (e.g., using contrast agents which create local variations in tissue elastic modulus or density) may be more difficult. While the theoretical (diffraction-limited) and currently practical resolutions of $100 \mathrm{MHz}$ ultrasound are $\sim 15 \mu \mathrm{m}$, and 15-60 $\mu \mathrm{m}$ (Foster et al., 2000), respectfully, at these frequencies, power is attenuated by brain tissue with a coefficient of $\sim 50 \mathrm{~dB} / \mathrm{cm}$ (Hoskins, 2010) $\left(10^{5}\right.$-fold attenuation per $\left.\mathrm{cm}\right)$, which imposes a penetration limit [e.g., for measurements with a dynamic range of $80 \mathrm{~dB}$ (Foster et al., 2000)]. Attenuation of ultrasound by bone is stronger still, at $22 \mathrm{~dB} /(\mathrm{cm} \mathrm{MHz})$ (Hoskins, 2010). Attenuation could therefore limit the use of ultrasound as a high-resolution neural recording modality in direct imaging modes, but multiplexed transmission of lower-frequency ultrasound from embedded devices could sidestep this issue.

\subsubsection{Conclusions and future directions}

Physics and information theory impose a tradeoff between bandwidth and power consumption in sending data through any communication channel. Considering only thermal noise and no path loss, achieving $100 \mathrm{Gbit} / \mathrm{s}$ data rates through a single channel necessitates either a bandwidth above a few $\mathrm{GHz}$ or a transmitted power above $\sim 100 \mathrm{~mW}$, the latter of which may be prohibitive from a heat dissipation perspective if the signals are to be generated by dissipative microelectronic devices. Researchers have proposed to use thousands or millions of tiny (Gómez-Martínez et al., 2010) wireless transmitters embedded in the brain to transmit local neural activity measurements to an external receiver via microwave radiation (Dyson, 2009). However, based on the above power-bandwidth tradeoff, this will require a bandwidth above a few $\mathrm{GHz}$. At the corresponding carrier frequencies, the penetration depth of the microwave radiation drops significantly, requiring increased power to combat the resulting signal loss. While one might hope that multiple independent channels could be multiplexed inside the brain, reducing the bandwidth and power requirements for each individual channel, the long wavelengths of microwave radiation compared to the mouse brain diameter suggest that such channels cannot be independent, as is confirmed by an analysis of the multi-input-multi-output (MIMO) channel capacity for this scenario. Therefore, radio-frequency electromagnetic transmission of whole brain activity data from embedded devices does not appear to be a viable option for brain activity mapping.

On the other hand, an analysis of the channel capacity for IR transmissions in a diffusive medium suggests that, because of its high frequency and decent penetration depth, infrared radiation may provide a viable substrate for transmitting activity data from embedded devices. For example, data could be transmitted via modulating the multiple-scattering speckle pattern of infrared light by varying the backscatter from an embedded optical device, such as an LCD pixel (Komanduri et al., 2008), in an activity-dependent fashion. Because the speckle pattern is sensitive to the motion of a single scatterer (Berkovits, 1991; Pappu et al., 2002), coherent multiple scattering could effectively act as an optical amplifier and as a means to create independent communication pathways. Furthermore, multiplexed data transmission via ultrasound is likely possible because of its short wavelength in tissue at reasonable carrier frequencies. It may also be of interest to explore network architectures (Bush, 2011) in 
which data is transmitted at low transmit power over short distances via local hops between neighboring nodes capable of signal restoration.

\subsection{MAGNETIC RESONANCE IMAGING}

Magnetic resonance imaging (MRI) uses the resonant behavior of nuclear spins in a magnetic field to non-invasively probe the spatiotemporally varying chemical and magnetic properties of tissues. Although originally conceived as a means to image anatomy, MRI can be used to observe neural activity provided that correlates of such activity are reflected in dynamic changes in local chemistry or magnetism.

In an MRI study, a strong static field ( $B=1-15 \mathrm{~T})$ is applied to polarize nuclear spins (usually ${ }^{1} \mathrm{H}$ ), causing them to resonate at a field-dependent Larmor frequency

$$
f=\frac{\gamma}{2 \pi} B
$$

where $\gamma$ is the gyromagnetic ratio of the nucleus (e.g., ${ }^{1} \mathrm{H}$ has a gyromagnetic ratio of $267.522 \mathrm{MHz} / \mathrm{T}$ (Mohr et al., 2010) and therefore resonates at $42.577 \mathrm{MHz}$ in a $1 \mathrm{~T}$ field). To obtain positional information, spatial field gradients are applied such that nuclei at different positions in the sample resonate at slightly different frequencies. Sequences of RF pulses and gradients are then applied to the sample, eliciting resonant emissions that contain information about spins' local chemical environment, magnetic field anisotropy and various other properties.

Most functional studies rely on dynamic changes in two forms of relaxation experienced by RF-excited spins. The first form results from energy dissipation through interactions with other species (e.g., other spins or unpaired electrons), causing the spins to recover their lowest energy state on a timescale, $T_{1}$, of 100 $1000 \mathrm{~ms}$ (Rooney et al., 2007). The second form of relaxation reflects the dephasing of spin signals in a given sampling volume (voxel) over a timescale, $T_{2}$, of 10-100 ms (Deichmann et al., 1995) due to non-uniform Larmor frequencies caused, e.g., by the presence of local magnetic field inhomogeneities.

In blood-oxygen level dependent (Ogawa et al., 1990) functional MRI (BOLD-fMRI), the most widely used form of neural $\mathrm{MR}$ imaging, increased neural activity in a given brain region alters the vascular concentration of paramagnetic deoxyhemoglobin, which affects local magnetic field homogeneity and thereby alters $T_{2}$. Although the existence of this paramagnetic reporter of oxygen metabolism is fortuitous, the data it provides is only an indirect readout of neural activity (Logothetis, 2008; Sirotin and Das, 2009; Jukovskaya et al., 2011), which is limited in its spatial and temporal resolution to the dynamics of blood flow in the brain's capillary network (1-2s). The spatial pointspread function of the hemodynamic BOLD response is in the $1 \mathrm{~mm}$ range, although sub-millimeter measurements, revealing cortical laminar and columnar features, have been obtained by filtering out the signals from larger blood vessels (Bandettini, 2009). A significant area of current and future work is aimed at developing new molecular reporters that can be introduced into the brain to transduce aspects of neural signaling such as calcium spikes and neurotransmitter release into MRI- detectable magnetic or chemical signals (Shapiro et al., 2010; Hsieh and Jasanoff, 2012; Koretsky, 2012), as described in section 4.5.3, below.

\subsubsection{Spatiotemporal Resolution}

The temporal resolution of MRI is limited by the dynamics of spin relaxation. For sequential MR signal acquisitions to be fully independent, spins must be allowed to recover their equilibrium magnetization on the timescale of $T_{1}(100-1000 \mathrm{~ms})$. However, if local $T_{1}$ is static its pre-mapping could enable temporally variant $T_{2}$ effects to be observed at refresh rates on the faster $T_{2}$ timescale (10-100 ms) (Deichmann et al., 1995). It may also be possible to detect events that occur on a timescale shorter than $T_{1}$ and $T_{2}$, if the magnitude of the resulting change in spin dynamics overcomes the lack of independence between acquisitions. Note that these limitations on the repetition time of the underlying pulse sequence are not eliminated by "fast" pulse sequences such as echo-planar imaging (EPI) (Stehling et al., 1991) and fast lowangle shot (FLASH) (Haase et al., 1986) or by the use of multiple detector coils (Wiesinger et al., 2006). These techniques accelerate the acquisition of $2 \mathrm{D}$ and $3 \mathrm{D}$ images, but still require spins to be prepared for readout.

The spatial resolution of current MRI techniques is limited by the diffusion of water molecules during the acquisition time (Glover and Mansfield, 2002), since contrast at scales above the diffusion length will be attenuated by diffusion. The RMS distance of a water molecule from its origin, after diffusing in $3 \mathrm{D}$ for a time $T_{\text {acq }}$, is

$$
d_{\mathrm{rms}}=\sqrt{6 D_{\mathrm{water}} T_{\mathrm{acq}}}
$$

where $D_{\text {water }}=2300 \mu \mathrm{m}^{2} / \mathrm{s}$ is the self-diffusion coefficient of water. For $T_{\mathrm{acq}} \approx 100 \mathrm{~ms}, d_{\mathrm{rms}} \approx 37 \mu \mathrm{m}$, which sets the approximate spatial resolution. For ultra-short acquisitions at $T_{\mathrm{acq}} \approx$ $10 \mathrm{~ms}, d_{\text {rms }} \approx 12 \mu \mathrm{m}$.

More technically, as described above, MRI uses field gradients to encode spatial positions in the RF frequency (wavenumber) components of the emitted radiation. The quality of the reconstruction of frequency space thus limits the achievable spatial resolution. The sampling interval of the detector $\Delta t$, and the field gradient $G$, determine the wavenumber increment as

$$
\Delta k=\gamma G \Delta t
$$

The spatial resolution (here considering only one dimension) is then given by (Glover and Mansfield, 2002):

$$
\Delta x_{k \text {-space }}=\frac{\pi}{\frac{T_{\mathrm{acq}}}{\Delta t} \Delta k}=\frac{\pi}{T_{\mathrm{acq}} \gamma G}
$$

Note that it is the gradient field, not the polarizing field $B_{0}$, which determines the resolution. For a gradient field of $100 \mathrm{mT} / \mathrm{m}$ and an acquisition time of $100 \mathrm{~ms}$

$$
\Delta x_{k \text {-space }}=\frac{\pi}{(100 \mathrm{~ms})(267 \mathrm{MHz} / \mathrm{T})(100 \mathrm{mT} / \mathrm{m})} \approx 1.17 \mu \mathrm{m}
$$

Due to relaxation, however, the emissions from a spin at a given position do not constitute a pure tone with a well-defined 
frequency. Instead, each spin exhibits a frequency spread, which gives rise to another limit on the spatial resolution (Glover and Mansfield, 2002):

$$
\Delta x_{\text {relaxation }}=\frac{2}{\gamma G T_{2}^{*}}
$$

where $T_{2}^{*}$ is the shortest relaxation time. Assuming $T_{2}^{*}=5 \mathrm{~ms}$ and $G=100 \mathrm{mT} / \mathrm{m}$, gives

$$
\Delta x_{\text {relaxation }} \approx 14 \mu \mathrm{m}
$$

Therefore, for water protons, the resolution limit is set by diffusion over $\sim 100 \mathrm{~ms}$ acquisition timescales, rather than by $k$-space sampling or relaxation. For other spin species (e.g., with lower diffusion rate), it may be possible to achieve resolutions limited by frequency discrimination.

Notably, there exists a practical trade-off between spatial resolution, temporal resolution, and sensitivity (SNR). In particular, to achieve high spatial resolution, it is necessary to densely sample $k$-space. Fast sampling sequences such as FLASH and EPI achieve speed by sampling each point of $k$-space using less signal and often at a lower resolution. Even at high field strengths $(11.7 \mathrm{~T})$, this tradeoff results in practical EPI-fMRI with a spatial resolution of $150 \times 150 \times 500 \mu \mathrm{m}$ and a temporal resolution of $200 \mathrm{~ms}$ (Yu et al., 2012). Achieving much higher spatial resolutions requires longer acquisitions and/or lower temporal sampling. For example, achieving a $20 \mu \mathrm{m}$ anatomical resolution in MRI of Drosophila embryos required $54 \mathrm{~min}$ for a small field of view of $2.5 \times 2.5 \times 5 \mathrm{~mm}$ (Null et al., 2008). Furthermore, the flies were administered paramagnetic gadolinium chelates to shorten $T_{1}$ and thereby the acquisition time. Separately, frame rates of $50 \mathrm{~ms}$ have been obtained for dynamic imaging of the human heart, but required the use of strong priors to reduce data collection requirements (Zhang et al., 2010).

\subsubsection{Energy dissipation}

Energy is dissipated into the brain when the excited spins relax to their equilibrium magnetization in the applied field. The energy associated with this relaxation is of order the Zeeman energy:

$$
\Delta E_{\text {Zeeman }}=\frac{\gamma}{2 \pi} h B_{0}
$$

To obtain an upper bound on the heat dissipation of MRI, we first assume that the brain is entirely water, that every proton spin is initially aligned by the field and then excited by the RF pulse, and that all spins relax during a $T_{1}$ relaxation time of $\sim 600 \mathrm{~ms}$. In this scenario, even an applied field of as high as $\sim 200 \mathrm{~T}$ would generate dissipation within the $\sim 50 \mathrm{~mW}$ energy dissipation limit. In reality, the energy dissipation is $4-5$ orders of magnitude smaller, because only a tiny fractional excess of the spins are initially aligned by the field $\left(\sim 1 \times 10^{-5}\right.$ for fields on the order of $\left.1 \mathrm{~T}\right)$. Therefore, thermal dissipation associated with spin excitation in MRI is unlikely to cause problems unless field strengths much greater than the largest currently used fields $(\sim 20 \mathrm{~T})$ are invoked, or spins with much higher gyromagnetic ratios are used.
Practically, the main energy consideration in MRI is the absorption by tissues of RF energy applied during imaging pulse sequences and the switching of magnetic field gradients. Such absorption is often calculated through numerical solutions of the Maxwell Equations taking into account the precise geometry, tissue properties and applied fields for a particular experimental setup (Collins et al., 2004). The typical specific absorption rate (SAR) is well under $10 \mathrm{~W} / \mathrm{kg}$ (or $5 \mathrm{~mW}$ per $500 \mathrm{mg}$ ), and is restricted by the FDA to less than $3 \mathrm{~W} / \mathrm{kg}$ for human studies.

\subsubsection{Imaging agents}

All the preceding discussion about spatiotemporal resolution presumes the existence of local time-varying signals (e.g., changes in $T_{1}$ or $T_{2}$ ) corresponding to the dynamics of neural activity. The hemodynamic BOLD response is the most prominent such signal, the limitations of which are discussed above. There have been studies working toward direct detection of minute (e.g., $\sim 0.2 \mathrm{nT})$ magnetic fields associated with action potentials through their effects on MRI phase or magnitude contrast (Bodurka and Bandettini, 2002; Petridou et al., 2006), but reliably detecting these fields above the physiological noise will likely require novel strategies (Witzel et al., 2008; Halpern-Manners et al., 2010) and estimates of the feasibility of these methods have been complicated by the lack of a realistic model for the local distribution of neuronal currents. MRI detection of the mechanical displacement of active neurons due to the Lorentz force in an applied magnetic field (Roth and Basser, 2009) has also been explored, as has the detection of activity-dependent changes in the diffusion of tissue water (Le Bihan et al., 2006; Tsurugizawa et al., 2013), possibly due to neuronal or glial (Kitaura et al., 2009) cell swelling (Holthoff and Witte, 1996; Isokawa, 2005), although strongly diffusion-weighted scans may have disadvantages in terms of SNR (Jasanoff, 2007). Manganese influx through voltage-gated calcium channels (Lin and Koretsky, 1997; Van der Linden et al., 2002) generates MRI contrast, but exhibits slow uptake kinetics and even slower efflux, such that manganese monotonically accumulates in the neurons over time. Conceivably, over-expression of manganese efflux pumps such as the iron transporter ferroportin (Madejczyk and Ballatori, 2012) could allow time-dependent activity imaging using manganese contrast.

In the past 15 years, efforts have been undertaken to develop chemical and biomolecular imaging agents that can be introduced into the brain to produce MRI detectable signals corresponding to specific aspects of neural function (analogously to fluorescent dyes and proteins). One critical advantage of using genetically encoded indicators would be the ability to target these indicators to specific cell types (Luo et al., 2008; Madisen et al., 2009) and/or cellular compartments (El-Husseini et al., 2001; Jacobs et al., 2003; Boeckers et al., 2005; Arnold, 2007; Feinberg et al., 2008; Vacher et al., 2008; Corrêa et al., 2009; Yamagata and Sanes, 2012). Notable examples of engineered molecular MRI contrast agents include $T_{1}$ and $T_{2}$ sensors of calcium (Li et al., 1999; Atanasijevic et al., 2006) and a $T_{1}$ sensor of neurotransmitter release (Shapiro et al., 2010). Depending on their mode of action, these imaging agents can provide temporal resolutions ranging from $10 \mathrm{~ms}$ to $10 \mathrm{~s}$ (Shapiro et al., 2006). However, a 
major current limitation for fast agents is the requirement that they be present in tissues at $\mu \mathrm{M}$ concentrations, posing major challenges for delivery and genetic expression. Model organisms lacking hemoglobin (e.g., the blowfly), and hence lacking a hemodynamic BOLD response (as is also the case for ex vivo brain slices), may be particularly useful for in vivo testing of novel activity-dependent contrast mechanisms, and specialized setups have been constructed to perform MRI at near-cellular spatial resolution in this context (though still requiring several hours to generate whole-brain anatomical images at this resolution) (Jasanoff and Sun, 2002).

Figure 6 shows the achievable temporal resolution for various classes of activity-dependent MRI contrast agents as well as the spatial resolution limit due to water proton diffusion.

\subsubsection{Conclusions and future directions}

Moving beyond hemodynamic contrast is crucial for improving the spatiotemporal resolution of $\mathrm{fMRI}$, and several avenues may be available for doing so, especially through the use of novel molecular contrast agents and/or genetic engineering. More fundamentally, current MRI techniques rely on the excitation of proton spins in water: this limits imaging to $>100 \mathrm{~ms}$ timescales, unless SNR is severely compromised, due to the low polarizability and long $T_{1}$ relaxation times of proton spins. There is also a spatial resolution limit of tens of microns over these timescales due to water's fast diffusion. Methods which couple neural activity to non-diffusible, highly polarized spins could, in principle, ameliorate this situation.

\subsection{MOLECULAR RECORDING}

An alternative to electrical, optical or MRI recording is the local storage of data in molecular substrates. Each neuron could be engineered to write a record of its own time-varying electrical activities onto a biological macromolecule, allowing off-line extraction of data after the experiment. Such systems could, in principle, be genetically encoded, and would thus naturally record from all neurons at the same time.

One proposed implementation of such a "molecular ticker tape" would utilize an engineered DNA polymerase with a $\mathrm{Ca}^{2+}$ sensitive or membrane-voltage-sensitive error-rate (Zamft et al.,
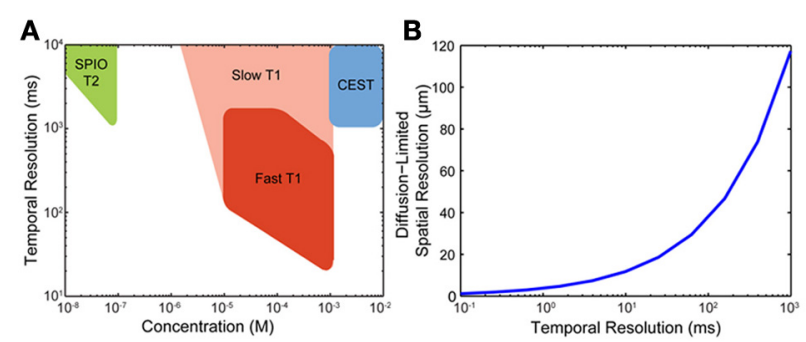

FIGURE 6 | Key factors determining the spatiotemporal resolution of dynamic MRI imaging. (A) Temporal resolution and contrast agent concentration allowing $>5 \%$ contrast, for different classes of dynamic MRI contrast agent [reproduced from Shapiro et al. (2006), with permission].

(B) Diffusion limited spatial resolution for water proton MRI as a function of temporal resolution.
2012) to record time-varying neural activities onto DNA (Glaser et al., 2013) as patterns of nucleotide misincorporations relative to a known template DNA strand [for alternative local recording techniques see (Friedland et al., 2009; Bonnet et al., 2013)]. The time-varying signal would later be recovered by DNA sequencing and subsequent statistical analysis (Glaser et al., 2013). DNA polymerases found in nature can add up to $\sim 1000$ nucleotides/s (Kelman and O'Donnell, 1995), and certain non-replicative polymerases such as DNA polymerase iota have error rates of $>70 \%$ on template $\mathrm{T}$ bases (Frank and Woodgate, 2007). Similar strategies could be implemented using RNA polymerases or potentially using other enzyme/hetero-polymer systems.

\subsubsection{Spatiotemporal resolution}

Polymerases proceed along their template DNA strands in a stochastic, thermally driven fashion; thus, polymerases that are initially synchronized will de-phase with respect to one another over time, occupying a range of positions on their respective templates at the time when a neural impulse occurs. The rate of this de-phasing is a key parameter governing the temporal resolution of molecular recording. By averaging over many simultaneously replicated templates, it is theoretically possible to associate variations in nucleotide misincorporation rate with the times at which these variations occurred, and thus to obtain temporally resolved recordings of the cation concentration (Glaser et al., 2013).

An analysis of the projected temporal resolution of molecular ticker tapes as a function of polymerase biochemical parameters can be found in (Glaser et al., 2013). This work suggests that molecular ticker tapes require synchronization mechanisms if they are to record at $<10 \mathrm{~ms}$ temporal resolution for durations longer than seconds, even when 10,000 templates per cell are recorded simultaneously, unless engineered polymerases with kinetic parameters beyond the limits of those found in nature can be developed. Recording at lower temporal resolutions, however, appears feasible using naturalistic biochemical parameters, even in the absence of synchronization mechanisms.

The development of mechanisms to improve synchronization of the ensemble of polymerases within each cell, or to encode time-stamps into the synthesized DNA (e.g., molecular clocks), could improve temporal resolution and decrease the number of required template strands per neuron. Mutation-based molecular clocks over evolutionary timescales are widely used in the field of phylogenetics (Ochman and Wilson, 1987), and new tools from synthetic biology (Elowitz and Leibler, 2000) and optogenetics or thermogenetics (Bernstein et al., 2012) also suggest strategies for building molecular clocks on faster timescales. As an example sketch of a possible synchronization mechanism, optogenetic methods [e.g., similar to (Konermann et al., 2013)] could be used to halt, and thus re-phase, a sub-population of polymerases at a light-dependent pause site in the template DNA, while another sub-population of polymerases reads through this pause site to maintain temporal continuity of recording; then the second population could be re-synchronized at an orthogonal light-dependent pause site while the first population reads 
through. Alternatively, some form of optogenetics could be used to directly write bit strings encoding time stamps into the synthesized DNA. These strategies would require one or two, sufficiently strong global clock signals to be optically broadcast to all neurons. The optics involved would be comparatively simple: this could be done using far fewer optical fibers than would be required for fiber-based activity readout, for instance. Alternatively, if the brain could be flash-frozen at a precisely known time, this could serve as a global time-stamp corresponding to the termination of DNA synthesis (e.g., the DNA $3^{\prime}$ end).

Spatial resolution for molecular recording would naturally reach the single cell level. To determine which nucleic acid tape originated from which neuron, static cell-specific DNA barcoding could be used (Zador et al., 2012) to associate the synthesized DNA strands with nodes in a topological connectome map obtained via DNA sequencing. Fluorescence in-situ DNA sequencing (FISSEQ) (Lee et al., submitted) on serially-sectioned or intact tissue (fixed post-mortem) (Chung et al., 2013) could be used to obtain explicit geometric information.

\subsubsection{Energy Dissipation}

4.6.2.1. Nucleotide metabolism. DNA polymerization imposes a metabolic load on the cell. Replication of the 3 billion bp human genome takes approximately $8 \mathrm{~h}$ in normally dividing cells, which equates to a nucleotide incorporation rate of $\sim 100 \mathrm{kHz}$. Therefore, in order not to exceed the metabolic rates associated with normal genome replication, molecular ticker tapes operating at $1 \mathrm{kHz}$ polymerization speed (Kelman and O'Donnell, 1995) would be limited to approximately 100 simultaneously replicated templates per cell. Even more recordings would be possible for RNA ticker tapes. The mammalian cell polymerizes at least $10^{11}$ NTPs per 16-h cell cycle (Jackson et al., 2000). Therefore, $\sim 1,700$ RNA tickertapes, each operating at $1 \mathrm{kHz}$, could be placed in a cell before generating a metabolic impact equal to that of the cell's baseline transcription rate. While these comparisons to baseline physiological levels are reasonable guidelines, it is likely that a neuron can support higher metabolic loads associated with larger numbers of templates. The maximal rate of neuronal aerobic respiration is $\sim 5 \mathrm{fmol}$ of ATP minute via oxidative respiration (see the section on bio-luminescence). Assuming $\sim 1$ ATP equivalent consumed per nucleotide incorporation, if neuronal metabolism were entirely dedicated to polymerization, it could support the incorporation of up to $6 \times 10^{9}$ nucleotides per minute, or $10^{5}$ simultaneously replicated DNA templates at $1 \mathrm{kHz}$.

4.6.2.2. Power dissipation. Normal DNA and RNA synthesis do not produce problematic energy dissipation and molecular tickertapes will likewise not be highly dissipative, at least in the regime where nucleic acid polymerization rates do not exceed those associated with genome replication or transcription.

\subsubsection{Volume displacement}

The nucleus of a neuron occupies $\sim 6 \%$ of a neuron's volume $\left((4 \mu \mathrm{m})^{3} /(10 \mu \mathrm{m})^{3}\right)$. Ticker tapes operating at $1 \mathrm{kHz}$ with 10,000 simultaneously replicated templates could record for $300 \mathrm{~s}$ before the total length of DNA synthesized equals the human genome length. In the case of RNA polymerase II-based transcription, $2.75 \mathrm{~h}$ of recording by 10000 recorders is required to reach the net transcript length in the cell. Therefore, with appropriate mechanisms to fold/pack the nucleic acids generated by molecular ticker tapes, they would not impose unreasonable requirements on cellular volume displacement over minutes to hours.

\subsubsection{Conclusions and Future Directions}

Molecular recording of neural activity has the advantages of inherent scalability, single-cell precision, and low energy and volume footprints. Making molecular recording work at temporal resolutions approaching $1 \mathrm{kHz}$, however, will require multiple new developments in synthetic biology, including protein engineering to create a fast polymerase $(>1 \mathrm{kHz})$ that strongly couples proxies for neural activity to nucleotide incorporation probabilities. Synchronization mechanisms would likely be required to perform molecular recording at singlespike temporal resolution. An attractive potential payoff for molecular approaches to activity mapping is the prospect of seamlessly combining-within a single brain-the readout of activity patterns with the readout of structural connectome barcodes (Mishchenko, 2010; Zador et al., 2012), transcriptional profiles (Lee et al., submitted) (e.g., to determine cell type) or other (epi-)genetic signatures (Sanjana et al., 2012) which are accessible via high-throughput nucleic acid sequencing.

\section{DISCUSSION}

We have analyzed the physical constraints on scalable neural recording for selected modalities of measurement, data storage, data transmission and power harvesting. Each analysis is based on assumptions - about the brain, device physics, or system architecture-which may be violated. Understanding these assumptions can point toward strategies to work around them, and in some cases we have suggested possible directions for such workarounds. Even valid assumptions about natural brains may be subject to modification through synthetic biology or external perturbation. For example, methods for rapidly removing heat from the brain could work around our assumptions about its natural cooling capacity, supporting a range of highly dissipative recording modalities. Likewise, assumptions about the necessary bandwidth for data transmission could be relaxed if some information is stored locally and read out after the fact.

In some cases, theoretical extensions of our first-order analyses could reveal important insights. The power-bandwidth tradeoffs identified in section 4.4 for electromagnetic data transmission may place limits on the informational throughput of fMRI, for example, or a realistic simulation of heat fluxes in the brain could reveal the true limits of power dissipation. In many other cases, new experiments will be required to move beyond crude estimates of feasibility.

The analysis of physical limits illustrates challenges and opportunities for technology development. While the opportunities can only be touched upon here, and some directions have been treated elsewhere (Alivisatos et al., 2012, 2013; Dean et al., 2013), we 
anticipate further analyses which could explore design spaces in detail. Here we briefly summarize a sampling of new directions suggested by our analysis.

\subsection{ELECTRICAL RECORDING}

The signal to noise ratio for a voltage sensing electrode imposes limits on the number of neurons per electrode from which signals can be detected and spike-sorted, likely requiring roughly one electrode per 100 neurons. To go beyond this, pure voltage sensing nodes could be augmented with the ability to directionally resolve distinct sources. For example, the 3D motion of a charged nanoparticle in an electric field, or of a dielectric nanoparticle in an electric field gradient, could be monitored at each recording site (Wood, 2013).

\subsection{OPTICAL RECORDING}

While light scattering creates severe limitations on optical imaging, embedded optical microscopies could overcome these limits. Embedded optical imaging systems with high signal multiplexing capacity would be desirable, to minimize the required number and size of implanted optical probes.

One option might be to use time-of-flight information to multiplex many sensor readouts into a single optical fiber: this could potentially be realized using time-domain reflectometry techniques, commonly used to determine the positions of defects in optical fibers, coupled to neural activity sensors arranged along the fiber, which would modulate the fiber's local absorption or backscatter (Wood, 2013). Time-domain reflectometry techniques have already reached $40 \mu \mathrm{m}$ resolution (Lamy and Fontaine, 1981).

Alternatively, novel fluorescent or bio-luminescent activity indicators could in principle relax the limits associated with light scattering, either by enabling efficient two-photon excitation at lower light dosages, or through all-infrared imaging schemes. Infrared bio-luminescence may be a particularly highvalue target.

\subsection{DELIVERY}

For both embedded optical and electrical recording strategies, new delivery mechanisms will be needed to scale to whole mammalian brains. Many of the basic parameters for scalable delivery mechanisms are still unknown. For example, can a large number of ultra-thin nano-wire electrodes or optical fibers be delivered via the capillary network? Can cells such as macrophages engulf ultra-miniaturized microchips and transport them into brain tissue? Can the blood brain barrier be locally opened [e.g., using ultrasonic stimulation (Hynynen et al., 2005)] to allow targeted delivery of recording probes?

\subsection{INTRINSIC SIGNALS}

The ideal technique would not require exogenous contrast agents or genetically encoded indicators, instead relying on signals intrinsic to neurophysiology. Neurons exhibit few-nano-meter scale (Iwasa et al., 1980) membrane displacements (e.g., in response to Maxwell stresses from large local electric field variations) during the action potential (Oh et al., 2012). These can be measured using optical interferometry (Fang-Yen et al., 2004), but in principle they could also be monitored acoustically [and related activity-associated membrane swellings have been directly observed by atomic force microscopy (Kim et al., 2007) in cultured neurons]. Sensors could be embedded in or around tissue to transduce the resulting acoustic vibrations into an electrical or optical readout. This could potentially allow recording at larger distances than the $\sim 130 \mu \mathrm{m}$ maximum recording radius for a voltage sensing node. Other intrinsic signals include changes in refractive index associated with neural activity, which will modulate the reflection and scattering of light (Stepnoski et al., 1991). These intrinsic changes in optical properties can be measured with optical coherence tomography (OCT) (Lazebnik et al., 2003). Local metabolic and hemodynamic signatures are also detectable optically, such as hemoglobin oxygenation [e.g., via functional near-infrared spectroscopy (Hoshi, 2003)] and the partial pressure of oxygen (Lecoq et al., 2011; Parpaleix et al., 2013). For minimal invasiveness, diffuse optical tomography uses near-infrared light (600-950 nm), which passes sufficientlyreadily through the skin and skull to allow imaging of hemodynamics in cortex (Joseph et al., 2006; Hillman, 2007; Huppert et al., 2009), although currently with limited spatial and temporal resolution.

\subsection{DATA TRANSMISSION THROUGH DIFFUSIVE MEDIA}

Unlike radio-frequency electromagnetics, infrared wavelengths may allow spatially multiplexed data transmissions from embedded recording devices, creating multiple independent channels by taking advantage of the stochasticity of light paths in strongly-scattering tissue. Alternatively, techniques are emerging to dynamically measure and invert the optical scattering matrix of a turbid medium, using pure-optical or hybrid techniques.

\subsection{ULTRASOUND}

Certain wavelengths of ultrasound exhibit potentially-favorable combinations of wavelength (spatial resolution), bandwidth (frequency) and attenuation compared to radio-frequency electromagnetics. Ultrasound could be used as a mechanism for powering and communicating with embedded local recording chips (Seo et al., 2013). Novel indicators (Shapiro, In revision) would likely need to be developed to perform neural activity imaging using pure ultrasound. Hybrid techniques such as photo-acoustic (Filonov et al., 2012) or ultrasound-encoded optical (Wang et al., 2012) microscopies are also of interest.

\subsection{MOLECULAR RECORDING}

For local recording, molecular recording devices could sidestep power constraints on embedded electronics, at the cost of increased engineering complexity. For molecular recording to become practical at temporal resolutions approaching the millisecond scale, sophisticated protein and viral engineering would likely be required to create a high-speed polymerase-based recorder operating in the neuronal cytoplasm. This would also necessitate molecular synchronization or time-stamping mechanisms to maintain phasing between multiple polymerases within a single cell, as well as between different cells.

On the other hand, molecular recording devices operating at slower timescales (e.g., seconds) could perhaps be engineered 
via more conservative combinations of known mechanisms, such as CREB-mediated signaling to the nucleus (Deisseroth, 2012) or nuclear-localized calcium sensing (Schrödel et al., 2013). In either case, the nucleic acid strands resulting from such molecular recorders could be space-stamped with cell-specific viral connectome barcodes (Zador et al., 2012) for later readout by bulk sequencing. Alternatively, the ticker tapes could be read within their anatomical contexts by in-situ sequencing, i.e., nucleic acid sequencing performed inside intact tissue (Lee et al., submitted).

\subsection{COMBINING STATIC AND DYNAMIC DATASETS}

Combining dynamic activity information with static structural or molecular information could allow these datasets to disambiguate one another. For example, a diversity of colors for fluorescent activity indicators (i.e., a form of BrainBow (Livet et al., 2007) calcium imaging) could ease requirements on spatial separation of optical signals, and the color pattern across cells could be mapped post-mortem at single-cell resolution using in-situ microscopy. Generalizing further, in-situ sequencing enables the extraction of vast quantities of molecular data from fixed tissue, in effect allowing observations with a palette of $4^{N}$ colors, where $N$ is the length of the nucleic acid polymer. It may be possible to harness this exponential informational resource to enhance the readout of dynamic activity information as well, e.g., through molecular recording.

\subsection{MRI}

Current MRI is limited by its reliance on intrinsic hemodynamic contrast mechanisms and on rapidly diffusing aqueous protons. Indicators coupling neural activity to spin relaxation rates are being developed to move beyond hemodynamic contrast. Novel excitation and detection schemes that could sensitize MRI to fast, local, intrinsically activity-dependent mechanisms (e.g., cell swelling, neuronal magnetic fields), while filtering out the slower BOLD response, are also of interest and should initially be tested in organisms or slice preparations lacking hemodynamic responses. Detailed computational models of neuronal currents within a tissue voxel [e.g., in the spirit of (Reimann et al., 2013)], and of the resulting mechanical and chemical changes, could be useful for evaluating potential new methods. In principle, MRI could also abandon the use of water protons as the signal sources, although this would pose significant implementation challenges.

\subsection{READOUT METHODS}

New signal processing frameworks such as compressive sensing could reduce bandwidth requirements and inspire new microscope designs exploiting computational imaging principles (Raskar and Tumblin, 2009; Kim, 2010; Velten et al., 2012; Pnevmatikakis and Paninski, 2013). Fast readout mechanisms (Lauxtermann et al., 2001) applied to giga-pixel arrays (e.g., the 3.2 giga-pixel CCD camera planned for the Large Synoptic Survey Telescope, which will have $\sim 1 \mathrm{~s}$ readout time) might be adapted to large-scale electrical or optical recording methods. Linear photodiode arrays can achieve $70 \mathrm{kHz}$ line readout rates (Reticon Inc., 2013), and many such linear arrays could be read out in parallel. Optoelectronic methods that convert between time, space and frequency representations of signals
(Goda et al., 2008, 2009b,a, 2012; Tsia et al., 2010; Mahjoubfar et al., 2011; Goda and Jalali, 2013) could inspire designs for even faster readouts (e.g., $\sim 10 \mathrm{MHz}$ frame rates have been demonstrated in brightfield imaging). Although these methods are not directly compatible with fluorescence measurements due to their use of spectral dispersion, related ideas (e.g., beat frequency multiplexing) may enable fluorescence microscopy at rates above that of CCD-based imaging (Diebold et al., 2013; Ducros et al., 2013), limited ultimately by fluorescence lifetimes, while also exhibiting favorable properties with respect to scattering.

\subsection{ALTERNATIVE MODALITIES}

X-ray imaging has been used on live cells (Moosmann et al., 2013) and might find use in neural recording if suitable contrast agents could be devised. X-rays interact with electron shells via photoelectric absorption and Compton scattering and with band structure in materials. X-ray phosphors utilize substitutions in an ionic lattice to generate visible or UV light emission upon Xray absorption (Issler and Torardi, 1995). In principle, some of these mechanisms could be engineered as neural activity sensors, e.g., in an absorption-contrast mode suitable for tomographic reconstruction (Larabell and Le Gros, 2004). While tissue damage due to ionizing radiation would ultimately be prohibitive [e.g., on a timescale of minutes (Wood, 2013)], very brief experiments might still be possible.

Likewise, electron spin resonance (ESR) operates at $\sim 100 \times$ higher Larmor frequency compared to proton MRI, which improves polarizability of the spins. Due to Pauli exclusion, use of this technique requires an indicator with unpaired electrons. These can be found in nitrogen vacancy diamond nano-crystals (Horowitz et al., 2012) (nano-diamonds), which are also sensitive to voltage (Dolde et al., 2011) and to magnetic fields (Hall et al., 2012), and are amenable to optical control and fluorescent readout of the spin state [although the $2 \mathrm{P}$ cross-section of the $(N-V)^{-}$center appears to be relatively low (Wee et al., 2007)].

\subsection{HYBRID SYSTEMS}

New mergers of input, sensing, and readout modalities can work around complex engineering constraints. Electrical or acoustic sensors could be used with optical (Sadek et al., 2010) (e.g., fiber) or ultrasonic readouts and power supplies. An MRI machine could interact with embedded electrical circuits powered by neural activity (Jasanoff, 0000). Linking electrical recording with embedded optical microscopies or other spatially-resolved methods could circumvent the limits of purely electrical spike sorting. Optical techniques such as holography or 4D light fields could generalize to ultrasound or microwave implementations. Consideration of analogies and synergies between fields suggests a combinatorial space of possibilities.

Our goal here has not been to pick winning technologies (which may not yet have been conceived), but to aid a multidisciplinary community of researchers in analyzing the problem. The challenge of observing the real-time operation of entire mammalian brains requires a return to first principles, and a fundamental reconsideration of the architectures of neural recording systems. We hope that knowledge of the constraints governing 
scalable neural recording will enable the invention of entirely new, transformative approaches.

\section{ACKNOWLEDGMENTS}

We thank K. Esvelt for helpful discussions on bioluminescent proteins; D. Boysen for help on the fuel cell calculations; R. Tucker and E. Yablonovitch (http://www.e3s-center.org) for helpful discussions on the energy efficiency of CMOS; C. Xu and C. Schaffer for data on optical attenuation lengths; T. Dean and the participants in his CS379C course at Stanford/Google, including Chris Uhlik and Akram Sadek, for helpful discussions and informative content in the discussion notes (http://www.stanford.edu/class/ cs379c/); and L. Wood, R. Koene, S. Rezchikov, A. Bansal, J. Lovelock, A. Payne, R. Barish, N. Donoghue, J. Pillow, W. Shih, P. Yin and J. Hewitt for helpful discussions and feedback on

\section{REFERENCES}

Ahmad, S., and Akdoan, E. K. (eds.) (2008). Piezoelectric and Acoustic Materials for Transducer Applications. Boston, MA: Springer. Available online at: http://www. amazon.com/dp/0387765387

Abrahamsson, S., Chen, J., Hajj, B., Stallinga, S., Katsov, A. Y., Wisniewski, J., et al. (2012). Fast multicolor 3d imaging using aberration-corrected multifocus microscopy. Nat. Methods 10, 60-63. doi: 10.1038/nmeth.2277

Ahrens, M. B., Orger, M. B., Robson, D. N., Li, J. M., and Keller, P. J. (2013). Whole-brain functional imaging at cellular resolution using light-sheet microscopy. Nat. Methods 10, 413-420. doi: 10.1038/nmeth.2434

Akemann, W., Sasaki, M., Mutoh, H., Imamura, T., Honkura, N., and Knöpfel, T. (2013). Two-photon voltage imaging using a genetically encoded voltage indicator. Sci. Rep. 3. doi: $10.1038 /$ srep02231

Alivisatos, A. P., Andrews, A. M., Boyden, E. S., Chun, M., Church, G. M., Deisseroth, K., et al. (2013). Nanotools for neuroscience and brain activity mapping. ACS Nano 7, 1850-1866. doi: $10.1021 / \mathrm{nn} 4012847$

Alivisatos, A. P., Chun, M., Church, G. M., Greenspan, R. J., Roukes, M. L., and Yuste, R. (2012). The brain activity map project and the challenge of functional connectomics. Neuron 74, 970-974. doi: 10.1016/j.neuron.2012.06.006

Allen, J. S., Damasio, H., and Grabowski, T. J. (2002). Normal neuroanatomical variation in the human brain: an MRI-volumetric study. Am. J. Phys. Anthropol. 118, 341-358. doi: 10.1002/ajpa.10092

Anastassiou, C. A., Buzsaki, C., and Koch, C. (2013). "Biophysics of extracellular spikes," in Principles of Neural Coding, eds R. Q. Quiroga and S. Panzeri (Boca Raton, FL: CRC Press), 15.

Anastassiou, C. A., Montgomery, S. M., Barahona, M., Buzsáki, G., and Koch, C. (2010). The effect of spatially inhomogeneous extracellular electric fields on neurons. J. Neurosci. 30, 1925-1936. doi: 10.1523/JNEUROSCI.3635-09.2010

Arnold, D. B. (2007). Polarized targeting of ion channels in neurons. Pflügers Archiv 453, 763-769. doi: 10.1007/s00424-006-0155-5

Atanasijevic, T., Shusteff, M., Fam, P., and Jasanoff, A. (2006). Calciumsensitive MRI contrast agents based on super paramagnetic iron oxide nanoparticles and calmodulin. Proc. Natl. Acad. Sci. U.S.A. $103,14707-14712 . \quad$ doi: 10.1073/pnas.0606749103

Azevedo, F. A. C., Carvalho, L. R. B., Grinberg, L. T., Marcelo Farfel, J., Ferretti, R. E. L., Leite, R. E. P., et al. (2009). Equal numbers of neuronal and nonneuronal cells make the human brain an isometrically scaled-up primate brain. $J$. Comp. Neurol. 513, 532-541. doi: 10.1002/cne.21974

Babadi, B., and Abbott, L. F. (2013). Pairwise analysis can account for network structures arising from spike-timing dependent plasticity. PLoS Comput. Biol. 9:e1002906. doi: 10.1371/journal.pcbi.1002906

Bakkum, D. J., Frey, U., Radivojevic, M., Russell, T. L., Müller, J., Fiscella, M. et al. (2013). Tracking axonal action potential propagation on a highdensity microelectrode array across hundreds of sites. Nat. Commun. 4. doi: $10.1038 /$ ncomms 3181

Bakopoulos, P., Karanasiou, I. S., Pleros, N., Zakynthinos, P., Uzunoglu, N., and Avramopoulos, H. (2009). A tunable continuous

earlier drafts. A. Marblestone is supported by the Fannie and John Hertz Foundation fellowship. D. Dalrymple is supported by the Thiel Foundation. K. Kording is funded in part by the Chicago Biomedical Consortium with support from the Searle Funds at The Chicago Community Trust. E. Boyden is supported by the National Institutes of Health (NIH), the National Science Foundation, the MIT McGovern Institute and Media Lab, the New York Stem Cell Foundation Robertson Investigator Award, the Human Frontiers Science Program, and the Paul Allen Distinguished Investigator in Neuroscience Award. B. Stranges, B. Zamft, R. Kalhor and G. Church acknowledge support from the Office of Naval Research and the NIH Centers of Excellence in Genomic Science. M. Shapiro is supported by the Miller Research Institute, the Burroughs Wellcome Career Award at the Scientific Interface and the W.M. Keck Foundation.

wave $(\mathrm{CW})$ and short-pulse optical source for $\mathrm{THz}$ brain imaging applications. Meas. Sci. Technol. 20:e104001. doi: 10.1088/09570233/20/10/104001

Bandettini, P. A. (2009). "Functional mri limitations and aspirations," in Neural Correlates of Thinking, eds E. Kraft, B. Gulyás, and E. Pöppel (Berlin: Springer), 15-38.

Bansal, A. K., Truccolo, W., VargasIrwin, C. E., and Donoghue, J. P. (2012). Decoding 3-d reach and grasp from hybrid signals in motor and premotor cortices: spikes, multiunit activity and local field potentials. J. Neurophysiol. 107, 1337-1355. doi: 10.1152/jn.00781.2011

Barnett, L., Platisa, J., Popovic, M., Pieribone, V. A., and Hughes, T. (2012). A fluorescent, geneticallyencoded voltage probe capable of resolving action potentials. PLoS ONE 7:e43454. doi: 10.1371/journal.pone.0043454

Barth, A. L., and Poulet, J. F. (2012). Experimental evidence for sparse firing in the neocortex. Trends Neurosci. $\quad 35, \quad 345-355 . \quad$ doi: 10.1016/j.tins.2012.03.008

Bédard, C., Kröger, H., and Destexhe, A. (2004). Modeling extracellular field potentials and the frequency-filtering properties of extracellular space. Biophys. J. 86, 1829-1842. doi: 10.1016/S00063495(04)74250-2

Bennett, C. H. (1973). Logical reversibility of computation. IBM J. Res. Dev. 17, 525-532. doi: 10.1147/rd.176.0525

Berkovits, R. (1991). Sensitivity of the multiple-scattering speckle pattern to the motion of a single scatterer. Phys. Rev. B 43, 8638-8640. doi: 10.1103/PhysRevB.43.8638

Bernstein, J. G., Garrity, P. A., and Boyden, E. S. (2012). Optogenetics and thermogenetics: technologies for controlling the activity of targeted cells within intact neural circuits. Curr. Opin. Neurobiol. 22, 61-71. doi: 10.1016/j.conb.2011. 10.023

Bertolotti, J., van Putten, E. G., Blum, C., Lagendijk, A., Vos, W. L., and Mosk, A. P. (2012). Non-invasive imaging through opaque scattering layers. Nature 491, 232-234. doi: $10.1038 /$ nature 11578

Bett, A. W., Dimroth, F., Löckenhoff, R., Oliva, E., and Schubert, J. (2008). "III-V Solar Cells Under Monochromatic Illumination," in Photovoltaic Specialists Conference, 2008, (San Diego, CA), 1-5.

Biederman, W., Yaeger, D. J., Narevsky, N., Koralek, A. C., Carmena, J. M., Alon, E., et al. (2013). A fully-integrated, miniaturized $\left(0.125 \mathrm{~mm}^{2}\right) \quad 10.5 \mu \mathrm{W}$ wireless neural sensor. IEEE J. SolidState Circuits 48, 960-970. doi: 10.1109/JSSC.2013.2238994

Blanchard, R., Boriskina, S. V., Genevet, P., Kats, M. A., Tetienne, J.-P., Yu, N., et al. (2011). Multiwavelength mid-infrared plasmonic antennas with single nanoscale focal point. Opt. Express 19, 22113-22124. doi: 10.1364/OE.19. 022113

Blinder, P., Tsai, P. S., Kaufhold, J. P., Knutsen, P. M., Suhl, H., and Kleinfeld, D. (2013). The cortical angiome: an interconnected vascular network with noncolumnar patterns of blood flow. Nat. Neurosci. 16, 889-897. doi: 10.1038/nn.3426

Bodurka, J., and Bandettini, P. A. (2002). Toward direct mapping of neuronal activity: Mri detection of ultraweak, transient magnetic field changes. Magn. Reson. Med. 47, 1052-1058. doi: 10.1002/mrm.10159 
Boeckers, T. M., Liedtke, T., Spilker, C., Dresbach, T., Bockmann, J., Kreutz, M. R., et al. (2005). C-terminal synaptic targeting elements for postsynaptic density proteins prosap $1 /$ shank 2 and prosap2/shank3. J. Neurochem. 92, 519-524. doi: 10.1111/j.1471-4159. 2004.02910.x

Bonnet, J., Yin, P., Ortiz, M. E., Subsoontorn, P., and Endy, D. (2013). Amplifying genetic logic gates. Science 340, 599-603. doi: 10.1126/science. 1232758

Bozinovic, N., Yue, Y., Ren, Y., Tur, M., Kristensen, P., Huang, H., et al. (2013). Terabit-scale orbital angular momentum mode division multiplexing in fibers. Science 340, 1545-1548. doi: $10.1126 /$ science. 1237861

Braitenberg, V., and Schüz, A. (1991). Anatomy of the Cortex: Statistics and Geometry. Berlin: Springer-Verlag Publishing.

Broxton, M., Grosenick, L., Yang, S., Cohen, N., Andalman, A., Deisseroth, K., et al. (2013). "Wave optics theory and 3-d deconvolution for the light field microscope," in Stanford Computer Graphics Laboratory Technical Report (Palo Alto, CA).

Bush, S. F. (2011). Toward in vivo nanoscale communication networks: utilizing an active network architecture. Front. Comput. Sci China 5, 316-326. doi: 10.1007/s11704-011-0116-9

Buzsáki, G., Anastassiou, C. A., and Koch, C. (2012). The origin of extracellular fields and currentsecog, Ifp and spikes. Nat. Rev. Neurosci. 13, 407-420. doi: 10.1038/ nrn3241

Buzsáki, G. (2004). Large-scale recording of neuronal ensembles. Nat. Neurosci. 7, 446-451. doi: 10.1038/nn1233

Campbell, A. K., and Herring, P. J. (1987). A novel red fluorescent protein from the deep sea luminous fish Malacosteus niger. Comp. Biochem. Physiol. B 86B, 411-417. doi: 10.1016/0305-0491(87)90314-2

Camuñas Mesa, L. A., and Quian Quiroga, R. (2013). A detailed and fast model of extracellular recordings. Neural Comput. 25, 1191-1212. doi: 10.1162/ NECO_a_00433

Cannon, G. J., and Swanson, J. A. (1992). The macrophage capacity for phagocytosis. J. Cell Sci. 101, 907-913.

Cao, G., Platisa, J., Pieribone, V. A., Raccuglia, D., Kunst, M., and Nitabach, M. N. (2013). Genetically targeted optical electrophysiology in intact neural circuits. Cell. doi: 10.1016/j.cell.2013.07.027

Carmena, J. M., Lebedev, M. A., Crist, R. E., O’Doherty, J. E., Santucci, D. M., Dimitrov, D. F., et al. (2003). Learning to control a brain-machine interface for reaching and grasping by primates. PLoS Biol. 1:e42. doi: 10.1371/journal.pbio.0000042

Carp, S. A., Roche-Labarbe, N., Franceschini, M.-A., Srinivasan, V. J., Sakadžić, S., and Boas, D. A. (2011). Due to intravascular multiple sequential scattering, diffuse correlation spectroscopy of tissue primarily measures relative red blood cell motion within vessels. Biomed. Opt. Express 2, 2047-2054. doi: 10.1364/BOE.2.002047

Castillo, M., Acevedo, P., and Moreno, E. (2003). KLM model for lossy piezoelectric transducers. Ultrasonics 41, 671-679. doi: 10.1016/S0041-624X(03)00101-X

Chadderton, P., Margrie, T. W., and Häusser, M. (2004). Integration of quanta in cerebellar granule cells during sensory processing. Nature 428, 856-860. doi: 10.1038 /nature02442

Chaigne, T., Katz, O., Boccara, A. C., Fink, M., Bossy, E., and Gigan, S. (2013). Controlling light in scattering media noninvasively using the photo-acoustic transmissionmatrix. eprint: arXiv:1305.6246

Cheng, A., Gonçalves, J. T., Golshani, P., Arisaka, K., and Portera-Cailliau, C. (2011). Simultaneous two-photon calcium imaging at different depths with spatiotemporal multiplexing. Nat. Methods 8, 139-142. doi: 10.1038/nmeth. 1552

Cho, N., Song, S.-J., Kim, S., Kim, S., and Yoo, H.-J. (2005). A 5.1- $\mu \mathrm{w}$ UHF RFID tag chip integrated with sensors for wireless environmental monitoring. In Solid-State Circuits Conference, 279-282.

Chung, K., Wallace, J., Kim, S.-Y., Kalyanasundaram, S., Andalman, A. S., Davidson, T. J., et al. (2013). Structural and molecular interrogation of intact biological systems. Nature 497, 332-337. doi: 10.1038/nature12107

Cinti, C., Taranta, M., Naldi, I., and Grimaldi, S. (2011). Newly engineered magnetic erythrocytes for sustained and targeted delivery of anti-cancer therapeutic compounds. PLoS ONE 6:e17132. doi: 10.1371/journal.pone.0017132

Collins, C. M., Liu, W., Wang, J., Gruetter, R., Vaughan, J. T., Ugurbil, K., et al. (2004). Temperature and SAR calculations for a human head within volume and surface coils at 64 and $300 \mathrm{MHz}$. J. Magn. Reson. Imaging 19, 650-656. doi: 10.1002/jmri.20041

Conkey, D. B., Caravaca-Aguirre, A. M., and Piestun, R. (2012). High-speed scattering medium characterization with application to focusing light through turbid media. Opt. Express 20, 1733-1740. doi: 10.1364/OE.20.001733

Corrêa, S. A., Müller, J., Collingridge, G. L., and Marrion, N. V. (2009). Rapid endocytosis provides restricted somatic expression of a $\mathrm{k}+$ channel in central neurons. J. Cell Sci. 122, 4186-4194. doi: $10.1242 /$ jcs. 058420

Costa, R. M., Cohen, D., and Nicolelis, M. A. (2004). Differential corticostriatal plasticity during fast and slow motor skill learning in mice. Curr. Biol. 14, 1124-1134. doi 10.1016/j.cub.2004.06.053

Cover, T. M., and Thomas, J. A. (2006). Elements of Information Theory. (Wiley Series in Telecommunications and Signal Processing, 2nd Edn. Hoboken, NJ: Wiley-Interscience

Dahan, M., Laurence, T., Pinaud, F., Chemla, D. S., Alivisatos, A. P., Sauer, M., et al. (2001). Time-gated biological imaging by use of colloidal quantum dots. Opt. Lett. 26, 825-827. doi: 10.1364/OL.26.000825

Dean, T., Ahanonu, B., Chowdhury, M., Datta, A., Esteva, A., Eth, D., et al. (2013). On the technology prospects and investment opportunities for scalable neuroscience. eprint: arXiv:1307.7302

Deichmann, R., Adolf, H., Nöth, U., Morrissey, S. P., Schwarzbauer, C. and Haase, A. (1995). Fast $T_{2}$ mapping with SNAPSHOT FLASH imaging. Magn. Reson. Imaging 13, 633-639. doi: 10.1016/0730-725X (95)00004-Z

Deisseroth，K. (2012). Optogenetics and psychiatry: applications, challenges, and opportunities. Biol. Psychiatry 71, 1030-1032. doi 10.1016/j.biopsych.2011.12.021

Derode, A., Tourin, A., de Rosny, J., Tanter, M., Yon, S., and Fink, M. (2003). Taking advantage of multiple scattering to communicate with time-reversal antennas. Phys. Rev. Lett. 90:e014301. doi: 10.1103/PhysRevLett.90.014301

Diebold, E. D., Buckley, B. W., Gossett, D. R., and Jalali, B. (2013). "Digitally-synthesized beat frequency multiplexing for sub-millisecond fluorescence microscopy," in Novel Techniques in Microscopy. Optical Society of America. Available online at: http://arxiv.org/abs/1303.1156
Dobbing, J., and Sands, J. (1973). Quantitative growth and development of human brain. Arch. Dis. Child. 48, 757-767. doi: 10.1136/adc.48.10.757

Dolde, F., Fedder, H., Doherty, M. W., Nöbauer, T., Rempp, F., Balasubramanian, G., et al. (2011). Electric-field sensing using single diamond spins. Nat. Phys. 7, 459-463. doi: 10.1038/nphys 1969

Dowski, E. R. Jr., and Cathey, W. T. (1995). Extended depth of field through wave-front coding. Appl. Opt. 34, 1859-1866. doi: 10.1364/AO.34.001859

Drobizhev, M., Makarov, N. S., Tillo, S. E., Hughes, T. E., and Rebane, A. (2011). Two-photon absorption properties of fluorescent proteins. Nat. Methods 8, 393-399. doi: 10.1038/nmeth.1596

Du, J., Blanche, T. J., Harrison, R. R., Lester, H. A., and Masmanidis, S. C. (2011). Multiplexed, high density electrophysiology with nanofabricated neural probes. PLoS ONE 6:e26204. doi: 10.1371/journal.pone.0026204

Ducros, M., Houssen, Y. G., Bradley, J., de Sars, V., and Charpak, S. (2013). Encoded multisite two-photon microscopy. Proc. Natl. Acad. Sci. U.S.A. 110, 13138-13143. doi: 10.1073/pnas.1307818110

Dyson, F. (2009). "Radiotelepathy, the direct communication of feelings and thought from brain to brain," in What Will Change Everything? ed J. Brockman, (Edge.org). Available online at: http://www. edge.org/q2009/q09_3.html\#dyson

El-Husseini, A. E.-D., Craven, S. E., Brock, S. C., and Bredt, D. S. (2001). Polarized targeting of peripheral membrane proteins in neurons. $J$. Biol. Chem. 276, 44984-44992. doi: 10.1074/jbc.M103049200

Elowitz, M. B., and Leibler, S. (2000). A synthetic oscillatory network of transcriptional regulators. Nature 403, 335-338. doi: $10.1038 / 35002125$

Engelhardt, B. (2006). Molecular mechanisms involved in $\mathrm{t}$ cell migration across the blood-brain barrier. J. Neural Trans. 113, 477-485. doi: 10.1007/s00702005-0409-y

Fang-Yen, C., Chu, M. C., Seung, H. S., Dasari, R. R., and Feld, M. S. (2004). Noncontact measurement of nerve displacement during action potential with a dual-beam low-coherence interferometer. Opt. Lett. 29, 2028-2030. doi: 10.1364/OL.29.002028

Fatouros, P. P., and Marmarou, A. (1999). Use of magnetic resonance 
imaging for in vivo measurements of water content in human brain: method and normal values. J. Neurosurg. $\quad 90$, 109-115. doi: 10.3171/jns.1999.90.1.0109

FDA. (2008). Guidance for Industry and fda Staff: Information for Manufacturers Seeking Marketing Clearance of Diagnostic Ultrasound Systems and Transducers. Available online at: http://www.fda.gov/ downloads/MedicalDevices/Device RegulationandGuidance/Guidance Documents/ucm070911.pdf

Fee, M. S., Mitra, P. P., and Kleinfeld, D. (1996). Variability of extracellular spike waveforms of cortical neurons. J. Neurophysiol. 76, 3823-3833.

Feinberg, E. H., VanHoven, M. K., Bendesky, A., Wang, G., Fetter, R. D., Shen, K., et al. (2008). Gfp reconstitution across synaptic partners (grasp) defines cell contacts and synapses in living nervous systems. Neuron 57, 353-363. doi: 10.1016/j.neuron.2007.11.030

Fiebig, M. (2005). Revival of the magnetoelectric effect. J. Phys. D Appl. Phys. 38:R123. doi: 10.1088/00223727/38/8/R01

Filonov, G. S., Krumholz, A., Xia, J., Yao, J., Wang, L. V., and Verkhusha, V. V. (2012). Deeptissue photoacoustic tomography of a genetically encoded nearinfrared fluorescent probe. Angew. Chem. 51, 1448-1451. doi: 10.1002/anie.201107026

Filonov, G. S., Platkevich, K. D., Ting, L.-M., Zhang, J., Kim, K., and Verkhusha, V. V. (2011). Bright and stable near-infrared fluorescent protein for in vivo imaging. Nat. Biotechnol. 29, 757-761. doi: 10.1038/nbt.1918

Flusberg, B. A., Cocker, E. D., Piyawattanametha, W., Jung, J. C., Cheung, E. L. M., and Schnitzer, M. J. (2005). Fiber-optic fluorescence imaging. Nat. Methods 2, 941-950. doi: 10.1038/nmeth820

Foster, F. S., Pavlin, C. J., Harasiewicz, K. A., Christopher, D. A., and Turnbull, D. H. (2000). Advances in ultrasound biomicroscopy. Ultrasound Med. Biol. 26, 1-27. doi: 10.1016/S0301-5629(99)00096-4

Frank, E. G., and Woodgate, R. (2007). Increased catalytic activity and altered fidelity of human DNA polymerase iota in the presence of manganese. J. Biol. Chem. 282, 24689-24696. doi: 10.1074/jbc.M702159200

Fraunhofer Institute for Photonic Microsystems. (2011). UHF/Microwave RFID Transponder ASIC IPMS_MWST1.
Fraunhofer Institute for Photonic Microsystems. Available online at: http://www.ipms.fraunhofer.de/cont ent/dam/ipms/common/products/W MS/Transponder/uhf-transponder-e. pdf (Accessed on June 16, 2013).

Freitas Jr., R. A. (1999). Nanomedicine, Volume I: Basic Capabilities. Georgetown, TX: Landes Bioscience.

Friedland, A. E., Lu, T. K., Wang, X., Shi, D., Church, G. M., and Collins, J. J. (2009). Synthetic gene networks that count. Science 324, 1199-1202. doi: 10.1126/science.1172005

Gabriel, S., Lau, R., and Gabriel, C. (1996). The dielectric properties of biological tissues: III. parametric models for the dielectric spectrum of tissues. Phys. Med. Biol. 41:2271. doi: 10.1088/0031-9155/41/11/003

Ge, D., Le Carpentier, E., Idier, J., and Farina, D. (2011). Spike sorting by stochastic simulation. IEEE Trans. Neural Syst. Rehabil. Eng. 19, 249-259. doi: 10.1109/TNSRE.2011.2112780

Gerhard, F., Kispersky, T., Gutierrez, G. J., Marder, E., Kramer, M., and Eden, U. (2013). Successful reconstruction of a physiological circuit with known connectivity from spiking activity alone. PLoS Comput. Biol. 9:e1003138. doi: 10.1371/journal.pcbi.1003138

Gershenfeld, N. (2000). The Physics of Information Technology. Cambridge: Cambridge University Press.

Gire, D. H., Restrepo, D., Sejnowski, T. J., Greer, C., De Carlos, J. A., and Lopez-Mascaraque, L. (2013). Temporal processing in the olfactory system: can we see a smell? Neuron 78, 416-432. doi: 10.1016/j.neuron.2013.04.033

Gittis, A. H., Moghadam, S. H., and du Lac, S. (2010). Mechanisms of sustained high firing rates in two classes of vestibular nucleus neurons: differential contributions of resurgent $\mathrm{Na}, \mathrm{Kv} 3$, and $\mathrm{BK}$ currents. J. Neurophysiol. 104, 1625-1634. doi: 10.1152/jn.00378.2010

Glaser, J. I., Zamft, B. M., Marblestone, A. H., Moffitt, J. R., Tyo, K., Boyden, E. S., et al. (2013). Statistical analysis of molecular signal recording. PLoS Comput. Biol. 9:e1003145. doi: 10.1371/journal.pcbi.1003145

Glover, P., and Mansfield, Sir P. (2002). Limits to magnetic resonance microscopy. Rep. Prog. Phys. 65, 1489-1511. doi: 10.1088/00344885/65/10/203

Goda, K., Ayazi, A., Gossett, D. R., Sadasivam, J., Lonappan, C. K., Sollier, E., et al. (2012). Highthroughput single-microparticle imaging flow analyzer. Proc. Natl. Acad. Sci. U.S.A. 109, 11630-11635. doi: 10.1073/pnas. 1204718109

Goda, K., and Jalali, B. (2013) Dispersive fourier transformation for fast continuous single-shot measurements. Nat. Photonics 7, 102-112. doi: 10.1038/nphoton. 2012.359

Goda, K., Solli, D. R., Tsia, K. K., and Jalali, B. (2009a). Theory of amplified dispersive fourier transformation. Physical Review A 80:043821. doi: 10.1103/PhysRevA.80.043821

Goda, K., Tsia, K., and Jalali, B. (2009b). Serial time-encoded amplified imaging for real-time observation of fast dynamic phenomena. Nature 458, 1145-1149. doi: $10.1038 /$ nature 07980

Goda, K., Tsia, K. K., and Jalali, B. (2008). Amplified dispersive fourier-transform imaging for ultrafast displacement sensing and barcode reading. Appl. Phys. Lett. 93, 131109-131109. doi: 10.1063/1.2992064

Goeppert-Mayer, M. (1931). Elementary processes with two quantum transitions. Ann. Phys. 18, 466-479. doi: 10.1002/andp.200910358

Gold, C., Henze, D. A., and Koch, C. (2007). Using extracellular action potential recordings to constrain compartmental models. J. Comput. Neurosci. 23, 39-58. doi: 10.1007/s10827-006-0018-2

Gómez-Martínez, R., Vázquez, P., Duch, M., Muriano, A., Pinacho, D., Sanvicens, N., et al. (2010). Intracellular silicon chips in living cells. Small 6, 499-502. doi: 10.1002/smll.200901041

Gong, Y., Li, J. Z., and Schnitzer, M. J. (2013). Enhanced archaerhodopsin fluorescent protein voltage indicators. PLoS ONE 8:e66959. doi: 10.1371/journal.pone.0066959

Gray, C. M., Maldonado, P. E., Wilson, M., and McNaughton, B. (1995). Tetrodes markedly improve the reliability and yield of multiple single-unit isolation from multiunit recordings in cat striate cortex. J. Neurosci. Methods 63 43-54. doi: 10.1016/0165-0270(95) 00085-2

Haase, A., Frahm, J., Matthaei, D. Hänicke, W., and Merboldt, K.-D. (1986). FLASH imaging. J. Magn. Reson. 67, 258-266.

Hall, L., Beart, G., Thomas, E., Simpson, D., McGuinness, L., Cole, J., et al. (2012). High spatial and temporal resolution wide-field imaging of neuron activity using quantum nv-diamond. Sci. Rep. 2.

Halpern-Manners, N. W., Bajaj, V. S., Teisseyre, T. Z., and Pines,
A. (2010). Magnetic resonance imaging of oscillating electrical currents. Proc. Natl. Acad. Sci. U.S.A. 107, 8519-8524. doi: $10.1073 /$ pnas. 1003146107

Harats, M. G., Schwarz, I., Zimran, A., Banin, U., Chen, G., and Rapaport, R. (2011). Enhancement of two photon processes in quantum dots embedded in subwavelength metallic gratings. Opt. Express 19, 1617-1625. doi: 10.1364/OE.19.001617

Harris, J. J., Jolivet, R., and Attwell, D. (2012). Synaptic energy use and supply. Neuron 75, 762-777. doi: 10.1016/j.neuron.2012.08.019

Henze, D. A., Borhegyi, Z., Csicsvari, J., Mamiya, A., Harris, K. D., and Buzsáki, G. (2000). Intracellular features predicted by extracellular recordings in the hippocampus in vivo. J. Neurophysiol. 84, 390-400.

Herculano-Houzel, S. (2011). Scaling of brain metabolism with a fixed energy budget per neuron: implications for neuronal activity, plasticity, and evolution. PLOS ONE 6:e17514. doi: 10.1371/journal. pone. 0017514

Higley, M. J., and Sabatini, B. L. (2008). Calcium signaling in dendrites and spines: practical and functional considerations. Neuron 59, 902-913. doi: 10.1016/j.neuron.2008.08.020

Hikawa, N., Horie, H., Kawakami, T., Okuda, K., and Takenaka, T. (1989). Introduction of macromolecules into primary cultured neuronal cells by fusion with erythrocyte ghosts. Brain Res. 481, 162-164. doi: 10.1016/0006-8993(89)90497-6

Hillman, E. M. (2007). Optical brain imaging in vivo: techniques and applications from animal to man. $J$. Biomed. Opt. 12, 051402-051402. doi: 10.1117/1.2789693

Ho, J., Kim, S., and Poon, A. (2013). Midfield wireless powering for implantable systems. Proc. IEEE 101, 1369-1378. doi: 10.1109/JPROC.2013.2251851

Holthoff, K., and Witte, O. W. (1996). Intrinsic optical signals in rat neocortical slices measured with near-infrared dark-field microscopy reveal changes in extracellular space. J. Neurosci. 16, 2740-2749.

Hopt, A., and Neher, E. (2001). Highly nonlinear photodamage in twophoton fluorescence microscopy. Biophys. J. 80, 2029-2036. doi: 10.1016/S0006-3495(01)76173-5

Hoskins, P. R., Martin, K., and Thrush, A. (eds.). (2010). Diagnostic Ultrasound: Physics and Equipment, 2nd Edn., New York, NY: Cambridge University Press. Available online 
at: http://www.amazon.com/dp/ 052175710X

Horowitz, V. R., Alemán, B. J., Christle, D. J., Cleland, A. N., and Awschalom, D. D. (2012). Electron spin resonance of nitrogen-vacancy centers in optically trapped nanodiamonds. Proc. Natl. Acad. Sci. U.S.A. 109, 13493-13497. doi: 10.1073/pnas.1211311109

Horton, N. G., Wang, K., Kobat, D., Clark, C. G., Wise, F. W., Schaffer, C. B., et al. (2013). In vivo three-photon microscopy of subcortical structures within an intact mouse brain. Nat. Photonics 7, 205-209. doi: 10.1038/nphoton. 2012.336

Hoshi, Y. (2003). Functional nearinfrared optical imaging: utility and limitations in human brain mapping. Psychophysiology 40, 511-520. doi: 10.1111/1469-8986.00053

Howarth, C., Gleeson, P., and Attwell, D. (2012). Updated energy budgets for neural computation in the neocortex and cerebellum. J. Cereb. Blood Flow Metab. 32, 1222-1232. doi: $10.1038 / \mathrm{jcbfm} .2012 .35$

Hromádka, T., DeWeese, M. R., and Zador, A. M. (2008). Sparse representation of sounds in the unanesthetized auditory cortex. PLoS Biol. 6:e16. doi: 10.1371/journal.pbio.0060016

Hsieh, V., and Jasanoff, A. (2012). Bioengineered probes for molecular magnetic resonance imaging in the nervous system. ACS Chem. Neurosci. 3, 593-602. doi: $10.1021 / \mathrm{cn} 300059 \mathrm{r}$

Huang, G., Jiang, H., Matthews, K., and Wilford, P. (2013). Lensless imaging by compressive sensing. In International Conference on Image Processing.

Huppert, T. J., Franceschini, M. A., and Boas, D. A. (2009). "14 noninvasive imaging of cerebral activation with diffuse optical tomography," in In Vivo Optical Imaging of Brain Function, 2nd Edn, ed R. D. Frostig (Boca Raton, FL: CRC Press).

Hynynen, K., McDannold, N., Sheikov, N. A., Jolesz, F. A., and Vykhodtseva, N. (2005). Local and reversible blood-brain barrier disruption by noninvasive focused ultrasound at frequencies suitable for trans-skull sonications. Neuroimage 24, 12-20. doi: 10.1016/j.neuroimage.2004.06.046

IEC. (2007). "IEC 60825: Safety of laser products," in Equipment Classification and Requirements, 2nd Edn., (Geneva: International Electrotechnical Commission), 6.

IEEE. (2006). C95.1-2005. IEEE Standard for Safety Levels with
Respect to Human Exposure to Radio Frequency Electromagnetic Fields, $3 \mathrm{khz}$ to $300 \mathrm{ghz}$. Available online at: $\mathrm{http} / /$ ieeexplore.ieee.org/xpl/login. jsp?tp $=$ \&arnumber $=1626482$

Iliff, J. J., Wang, M., Liao, Y., Plogg, B. A., Peng, W., Gundersen, G. A., et al. (2012). A paravascular pathway facilitates csf flow through the brain parenchyma and the clearance of interstitial solutes, including amyloid beta. Sci. Transl. Med. 4:147ral11. doi: 10.1126/scitranslmed.3003748

Impinj, Inc. (2012) Monza 5 Tag Chip Datasheet. Seattle, WA: Impinj, Inc., Available online at: http:// www.impinj.com/Documents/Tag Chips/Monza_5_Datasheet (Accessed on June 20,2013).

Ionescu, A. M., and Riel, H. (2011). Tunnel field-effect transistors as energy-efficient electronic switches. Nature 479, 329-337. doi: 10.1038 /nature10679

Isokawa, M. (2005). N-methyl$\mathrm{d}$-aspartic acid-induced and ca-dependent neuronal swelling and its retardation by brain-derived neurotrophic factor in the epileptic hippocampus. Neuroscience 131, 801-812. doi: 10.1016/j.neuro science.2004.12.008

Issler, S. L., and Torardi, C. C. (1995). Solid state chemistry and luminescence of X-ray phosphors. J. Alloys Compd. 229, 54-65. doi: 10.1016/ 0925-8388(95)01686-4

Ivanov, K. P., Kalinina, M. K., and Levkovich, Y. I. (1981) Blood flow velocity in capillaries of brain and muscles and its physiological significance. Microvasc. Res. 22, 143-155. doi: 10.1016/0026-2862 (81) $90084-4$

Iwasa, K., Tasaki, I., and Gibbons, R. C. (1980). Swelling of nerve fibers associated with action potentials. Science 210, 338-339. doi: 10.1126/science. 7423196

Jackson, D. A., Pombo, A., and Iborra, F. (2000). The balance sheet for transcription. FASEB J. 14, 242-254.

Jacobs, E. C., Bongarzone, E. R., Campagnoni, C. W., Kampf, K., and Campagnoni, A. T. (2003). Somarestricted products of the myelin proteolipid gene are expressed primarily in neurons in the developing mouse nervous system. Dev. Neurosci. 25, 96-104. doi: 10.1159/000072259

Jadhav, A. D., Aimo, I., Cohen, D., Ledochowitsch, P., and Maharbiz, M. M. (2012). "Cyborg eyes: microfabricated neural interfaces implanted during the development of insect sensory organs produce stable neurorecordings in the adult," in IEEE 25th International Conference on Micro Electro Mechanical Systems (Paris), 937-940.

Jasanoff, A. (2007). Bloodless fmri. Trends Neurosci. 30, 603-610. doi: 10.1016/j.tins.2007.08.002

Jasanoff, A. (NIH Grant 1R01NS076462-01). Noninvasive Imaging-Based Electrophysiology using Microelectronic Devices. Available online at: http:// projectreporter.nih.gov/project_infodescript on.cfm?aid $=8180844$

Jasanoff, A., and Sun, P. Z. (2002) In vivo magnetic resonance microscopy of brain structure in unanesthetized flies. J. Magn. Reson. 158, 79-85. doi: 10.1016/ S1090-7807(02)00063-0

Jensen, W., Hofmann, U. G., and Yoshida, K. (2003). "Assessment of subdural insertion force of single-tine microelectrodes in rat cerebral cortex," in Proceedings of the 25th Annual International Conference of the IEEE Engineering in Medicine and Biology Society, Vol. 3, 2168-2171.

Jin, X., and Costa, R. M. (2010). Start/stop signals emerge in nigrostriatal circuits during sequence learning. Nature 466, 457-462. doi: 10.1038/nature09263

Jonasz, M. (2007). Absorption coefficient of water: data sources. Topics in Particle and Dispersion Science.

Joseph, D. K., Huppert, T. J., Franceschini, M. A., and Boas, D. A. (2006). Diffuse optical tomography system to image brain activation with improved spatial resolution and validation with functional magnetic resonance imaging. Appl. Opt. 45, 8142-8151. doi: 10.1364/AO.45 008142

Judkewitz, B., Wang, Y. M. Horstmeyer, R., Mathy, A., and Yang, C. (2013). Speckle-scale focusing in the diffusive regime with time reversal of variance-encoded light (trove). Nat. Photonics 7 , 300-305. doi: 10.1038/nphoton. 2013.31

Jukovskaya, N., Tiret, P., Lecoq, J., and Charpak, S. (2011). What does local functional hyperemia tell about local neuronal activation? J. Neurosci. 31, 1579-1582. doi: 10.1523/JNEURO SCI.3146-10.2011

Kadiu, I., Nowacek, A., McMillan, J., and Gendelman, H. E. (2011). Macrophage endocytic trafficking of antiretroviral nanoparticles. Nanomedicine 6, 975-994. doi: $10.2217 / \mathrm{nnm} .11 .27$
Kalmbach, A. S., and Waters, J. (2012). Brain surface temperature under a craniotomy. J. Neurophysiol. 108, 3138-3146. doi: 10.1152/jn.00557. 2012

Kandel, E. R., Markram, H., Matthews, P. M., Yuste, R., and Koch, C. (2013). Neuroscience thinks big (and collaboratively). Nat. Rev. Neurosci. 14, 659-664. doi: 10.1038/nrn3578

Kang, J. W., Kim, P., Amadeo Alonzo, C., Park, H., and Yun, S. H. (2010). Two-photon microscopy by wavelength-swept pulses delivered through single-mode fiber. Opt. Lett. 35, 181-183. doi: 10.1364/OL.35.000181

Karalis, A., Joannopoulos, J., and SoljaĆIĆ, M. (2008). Efficient wireless non-radiative mid-range energy transfer. Ann. Phys. 323, 34-48. doi: 10.1016/j.aop.2007.04.017

Katz, O., Small, E., Bromberg, Y., and Silberberg, Y. (2011). Focusing and compression of ultrashort pulses through scattering media. Nat. Photonics 5, 372-377. doi: 10.1038/nphoton.2011.72

Katz, O., Small, E., and Silberberg, Y. (2012). Looking around corners and through thin turbid layers in real time with scattered incoherent light. Nat. Photonics 6, 549-553. doi: 10.1038/nphoton.2012.150

Kelman, Z., and O'Donnell, M. (1995). DNA polymerase III holoenzyme. Ann. Rev. Biochem. 64, 171-200. doi: 10.1146/annurev.bi.64.070195. 001131

Kim, G., Kosterin, P., Obaid, A., and Salzberg, B. (2007). A mechanical spike accompanies the action potential in mammalian nerve terminals. Biophys. J. 92, 3122-3129. doi: 10.1529/biophysj.106.103754

Kim, J. (2010). Next generation CAT system. PhD thesis, Massachusetts Institute of Technology.

Kim, K. H., Buehler, C., and So, P. T. C. (1999). High-speed, twophoton scanning microscope. Appl. Opt. 38, 6004-6009. doi: 10.1364/AO.38.006004

Kitagawa, Y., Hiraoka, Y., Honda, T., Ishikura, T., Nakamura, H., and Kimura, T. (2010). Low-field magnetoelectric effect at room temperature. Nat. Mater. 9, 797-802. doi: $10.1038 /$ nmat 2826

Kitaura, H., Tsujita, M., Huber, V. J., Kakita, A., Shibuki, K. Sakimura, K., et al. (2009). Activitydependent glial swelling is impaired in aquaporin-4 knockout mice. Neurosci. Res. 64, 208-212. doi: 10.1016/j.neures.2009.03.002

Kobat, D., Durst, M. E., Nishimura, N., Wong, A. W., Schaffer, C. B. 
and $\mathrm{Xu}, \mathrm{C}$. (2009). Deep tissue multiphoton microscopy using longer wavelength excitation. Opt. Express 17, 13354-13364. doi: 10.1364/OE.17.013354

Kobat, D., Horton, N. G., and Xu, C. (2011). In vivo two-photon microscopy to 1.6-mm depth in mouse cortex. J. Biomed. Opt. 16:106014. doi: 10.1117/1.3646209

Kodandaramaiah, S. B., Franzesi, G. T., Chow, B. Y., Boyden, E. S., and Forest, C. R. (2012). Automated whole-cell patch-clamp electrophysiology of neurons in vivo. Nat. Methods 9, 585-587. doi: 10.1038/nmeth.1993

Komanduri, R. K., Oh, C., and Escuti, M. J. (2008). "Reflective liquid crystal polarization gratings with high efficiency and small pitch," in Photonic Devices + Applications (San Diego, CA: International Society for Optics and Photonics), 70500J. doi: 10.1117/12.795529

Konermann, S., Brigham, M. D., Trevino, A., Hsu, P. D., Heidenreich, M., Cong, L., et al. (2013). Optical control of mammalian endogenous transcription and epigenetic states. Nature 500, 472-476. doi: 10.1038/ nature 12466

König, K., So, P., Mantulin, W., and Gratton, E. (1997). Cellular response to near-infrared femtosecond laser pulses in two-photon microscopes. Opt. Lett. 22, 135-136. doi: 10.1364/OL.22.000135

Koomey, J. G., Berard, S., Sanchez, M., and Wong, H. (2011). Implications of historical trends in the electrical efficiency of computing. IEEE Annals Hist. Comput. 33, 46-54. doi: 10.1109/MAHC.2010.28

Koralek, A. C., Jin, X., Long II, J. D., Costa, R. M., and Carmena, J. M. (2012). Corticostriatal plasticity is necessary for learning intentional neuroprosthetic skills. Nature 483, 331-335. doi: 10.1038/nature10845

Kording, K. P. (2011). Of toasters and molecular ticker tapes. PLoS Comput. Biol. 7:e1002291. doi: 10.1371/journal.pcbi.1002291

Koretsky, A. P. (2012). Is there a path beyond BOLD? Molecular imaging of brain function. Neuroimage 62, 1208-1215. doi: 10.1016/j.neuroimage.2012.02.076

Kou, L., Labrie, D., and Chylek, P. (1993). Refractive indices of water and ice in the $0.65-$ to $2.5-\mu \mathrm{m}$ spectral range. Appl. Opt. 32, 3531-3540. doi: 10.1364/AO.32.00353

Kralj, J. M., Douglass, A. D., Hochbaum, D. R., Maclaurin, D., and Cohen, A. E. (2012). Optical recording of action potentials in mammalian neurons using a microbial rhodopsin. Nat. Methods 9, 90-95. doi: 10.1038/nmeth.1782

Krimholtz, R. S., Leedom, D. A., and Matthaei, G. L. (1970). New equivalent circuits for elementary piezoelectric transducers. Electron. Lett. 6, 398-399. doi: 10.1049/el: 19700280

Lamy, F., and Fontaine, J. (1981). Transparent Media Characterization using Subpicosecond Dye Laser. Available online at: http://cds.cern. ch/record/870056/files/p35.pdf

Landauer, R. W. (1961). Irreversibility and heat generation in the computing process. IBM J. Res. Dev. 5, 183-191. doi: 10.1147/rd.53.0183

Larabell, C. A., and Le Gros, M. A. (2004). X-ray tomography generates 3-D reconstructions of the yeast, Saccharomyces cerevisiae, at 60-nm resolution. Mol. Biol. Cell 15, 957-962. doi: 10.1091/mbc.E03-070522

Larson, D. R., Zipfel, W. R., Williams, R. M., Clark, S. W., Bruchez, M. P., Wise, F. W., et al. (2003). Watersoluble quantum dots for multiphoton fluorescence imaging in vivo. Science 300, 1434-1436. doi: 10.1126/science. 1083780

Lauxtermann, S., Israel, G., Seitz, P., Bloss, H., Ernst, J., Firla, H., et al. (2001). "A mega-pixel high speed cmos imager with sustainable gigapixel/sec readout rate," in IEEE Workshop on Charge-Coupled Devices and Advanced Image Sensor at the 2001 (Lake Tahoe, NV).

Lazebnik, M., Marks, D. L., Potgieter, K., Gillette, R., and Boppart, S. A. (2003). Functional optical coherence tomography for detecting neural activity through scattering changes. Opt. Lett. 28, 1218-1220. doi: 10.1364/OL.28.001218

Lazzi, G. (2005). Thermal effects of bioimplants. IEEE Eng. Med. Biol. Mag. 24, 75-81. doi: 10.1109/MEMB.2005.1511503

Le Bihan, D., Urayama, S.-i., Aso, T., Hanakawa, T., and Fukuyama, H. (2006). Direct and fast detection of neuronal activation in the human brain with diffusion mri. Proc. Natl. Acad. Sci. U.S.A. 103, 8263-8268. doi: 10.1073/pnas.060 0644103

Lecoq, J., Parpaleix, A., Roussakis, E., Ducros, M., Houssen, Y. G., Vinogradov, S. A., et al. (2011). Simultaneous two-photon imaging of oxygen and blood flow in deep cerebral vessels. Nat. Med. 17, 893-898. doi: 10.1038/nm.2394

Lennie, P. (2003). The cost of cortical computation. Curr. Biol. 13, 493-497. doi: 10.1016/S0960-9822 (03)00135-0
Levoy, M., Zhang, Z., and McDowall, I. (2009). Recording and controlling the $4 \mathrm{~d}$ light field in a microscope using microlens arrays. J. Microsc 235, 144-162. doi: 10.1111/j.13652818.2009.03195.x

Li, W.-h., Fraser, S. E., and Meade, T. J. (1999). A calcium-sensitive magnetic resonance imaging contrast agent. J. Am. Chem. Soc. 121, 1413-1414. doi: 10.1021/ja9837021

Lin, Y.-J., and Koretsky, A. P. (1997) Manganese ion enhances t1weighted mri during brain activation: an approach to direct imaging of brain function. Magn. Reson. Med. 38, 378-388. doi: 10.1002/mrm.1910380305

Liu, T.-J. K., Marković, D., Stojanović, V., and Alon, E. (2012). The relay reborn. IEEE Spectr. 49, 40-43. doi: 10.1109/MSPEC.2012. 6172808

Livet, J., Weissman, T. A., Kang, H., Draft, R. W., Lu, J., Bennis, R. A., et al. (2007). Transgenic strategies for combinatorial expression of fluorescent proteins in the nervous system. Nature 450, 56-62. doi: 10.1038/nature06293

Llinás, R. R., Walton, K. D., Nakao, M., Hunter, I., and Anquetil, P. A (2005). Neuro-vascular central nervous recording/stimulating system. Journal of Nanoparticle Research 7 , 111-127. doi: 10.1007/s11051-0053134-4

Loffredo, F., and Lee, R. T. (2008). Therapeutic vasculogenesis. Circ. Res. 103, 128-130. doi 10.1161/CIRCRESAHA.108.180604

Logothetis, N. K. (2008). What we can do and what we cannot do with fmri. Nature 453, 869-878. doi: 10.1038/nature06976

Luo, L., Callaway, E. M., and Svoboda, K. (2008). Genetic dissection of neural circuits. Neuron 57, 634-660. doi: 10.1016/j.neuron.2008.01.002

Madejczyk, M. S., and Ballatori, N (2012). The iron transporter ferroportin can also function as a manganese exporter. Biochimica et Biophysica Acta (BBA)Biomembranes 1818, 651-657. doi: 10.1016/j.bbamem.2011.12.002

Madisen, L., Zwingman, T. A., Sunkin, S. M., Oh, S. W., Zariwala, H. A., $\mathrm{Gu}$, H., et al. (2009). A robust and high-throughput cre reporting and characterization system for the whole mouse brain. Nat. Neurosci. 13, 133-140. doi: 10.1038/ nn.2467

Mahalati, R. N., Gu, R. Y., and Kahn, J. M. (2013). Resolution limits for imaging through multi-mode fiber. Opt. Exp. 21, 1656-1668. doi: 10.1364/OE.21.001656
Mahan, G. D., Engler, W. E., Tiemann, J. J., and Uzgiris, E. E. (1998). Ultrasonic tagging of light. Proc. Natl. Acad. Sci. U.S.A. 95, 14015-14019. doi: 10.1073/pnas.95.24.14015

Mahjoubfar, A., Goda, K., Ayazi, A., Fard, A., Kim, S. H., and Jalali, B. (2011). High-speed nanometerresolved imaging vibrometer and velocimeter. Appl. Phys. Lett. 98, 101107-101107. doi: $10.1063 / 1.3563707$

Mandal, S., and Sarpeshkar, R. (2007). Low-power CMOS rectifier design for RFID applications. IEEE Trans. Circ. Syst. 54, 1177-1188. doi: 10.1109/TCSI.2007.895229

Markram, H., Gerstner, W., and Sjöström, P. J. (2011). A history of spike-timing-dependent plasticity. Front. Syn. Neurosci. 3:4. doi: 10.3389/fnsyn.2011.00004

Marre, O., Amodei, D., Deshmukh, N. Sadeghi, K., Soo, F., Holy, T. E., et al. (2012). Mapping a complete neural population in the retina. J. Neurosci. 32, 14859-14873. doi: 10.1523/JNEUROSCI.0723-12.2012

Marshall, J. D., and Schnitzer, M. J. (2013). Optical strategies for sensing neuronal voltage using quantum dots and other semiconductor nanocrystals. ACS Nano 7, 4601-4609. doi: 10.1021/nn40 1410k

Martin, J.-R. (2008). In vivo brain imaging: fluorescence or bioluminescence, which to choose? J. Neurogenet. 22, 285-307. doi: 10.1080/01677060802298517

Martin, J.-R., Rogers, K. L., Chagneau, C., and Brûlet, P. (2007). In vivo bioluminescence imaging of $\mathrm{ca} 2+$ signalling in the brain of drosophila. PLOS ONE 2:e275. doi: 10.1371/journal.pone.0000275

Masters, B. R. (2006). Confocal Microscopy and Multiphoton Excitation Microscopy. Number PM161 in SPIE Press Monograph. Bellingham, WA: SPIE Publications doi: $10.1117 / 3.660403$

McCabe, D. J., Tajalli, A., Austin, D. R., Bondareff, P., Walmsley, I. A., Gigan, S., et al. (2011). Spatiotemporal focusing of an ultrafast pulse through a multiply scattering medium. Nat. Commun. 2:447. doi: 10.1038/ncomms1434

McElligott, J., and Melzack, R. (1967). Localized thermal changes evoked in the brain by visual and auditory stimulation. Exp. Neurol. 17, 293-312. doi: 10.1016/0014-4886 (67)90108-2

Mishchenko, Y. (2010). On optical detection of densely labeled synapses in neuropil and mapping 
connectivity with combinatorially multiplexed fluorescent synaptic markers. PLoS ONE 5:e8853. doi: 10.1371/journal.pone.0008853

Miya, T., Terunuma, Y., Hosaka, T., and Miyashita, T. (1979). Ultimate low-loss single-mode fibre at 1.55 um. Electron. Lett. 15, 106-108. doi: 10.1049/el:19790077

Mohr, P. J., Taylor, B. N., and Newell, D. B. (2010). CODATA recommended values of the fundamental physical constants: 2010. Rev. Modern Phys. 84, 1527-1605. doi: 10.1103/RevModPhys.84.1527

Molter, T. W., McQuaide, S. C., Suchorolski, M. T., Strovad, T. J., Burgess, L. W., Meldrum, D. R., et al. (2009). A microwell array device capable of measuring singlecell oxygen consumption rates. Sens. Actuators B 135, 678-686. doi: 10.1016/j.snb.2008.10.036

Moosmann, J., Ershov, A., Altapova, V., Baumbach, T., Prasad, M. S., LaBonne, C., et al. (2013). Xray phase-contrast in vivo microtomography probes new aspects of Xenopus gastrulation. Nature 497, 374-377. doi: 10.1038/nature12116

Moustakas, A. L., Baranger, H. U., Balents, L., Sengupta, A., and Simon, S. H. (2000). Communication through a diffusive medium. Science 287, 287-290. doi: 10.1126/science.287.5451.287

Murray, T. A., and Levene, M. J. (2012). Singlet gradient index lens for deep in vivo multiphoton microscopy. J. Biomed. Opt. 17:021106. doi: 10.1117/1.JBO.17.2.021106

Najafi, K., and Hetke, J. F. (1990). Strength characterization of silicon microprobes in neurophysiological tissues. IEEE Trans. Biomed. Eng. 37, 474-481. doi: 10.1109/10.55638

Naumann, E. A., Kampff, A. R., Prober, D. A., Schier, A. F., and Engert, F. (2010). Monitoring neural activity with bioluminescence during natural behavior. Nat. Neurosci. 13, 513-520. doi: 10.1038/nn.2518

Nguyen, Q.-T., Schroeder, L. F., Mank, M., Muller, A., Taylor, P., Griesbeck, O., et al. (2009). An in vivo biosensor for neurotransmitter release and in situ receptor activity. Nat. Neurosci. 13, 127-132. doi: 10.1038/nn.2469

Nicolelis, M. A. L., Dimitrov, D., Carmena, J. M., Crist, R., Lehew, G., et al. (2003). Chronic, multisite, multielectrode recordings in macaque monkeys. Proc. Natl. Acad. Sci. U.S.A. 100, 11041-11046. doi: 10.1073/pnas.1934665100

Nixon, M., Katz, O., Small, E., Bromberg, Y., Friesem, A. A., Silberberg, Y., et al. (2013).
Real-time wavefront-shaping through scattering media by all optical feedback. Nat. Photon. doi: 10.1038/nphoton.2013.248

Null, B., Liu, C. W., Hedehus, M. Conolly, S., and Davis, R. W. (2008). High-resolution, in vivo magnetic resonance imaging of Drosophila at 18.8 tesla. PLoS ONE 3:e2817. doi: 10.1371/journal.pone.0002817

Ochman, H., and Wilson, A. C. (1987). Evolution in bacteria: evidence for a universal substitution rate in cellular genomes. J. Mol. Evol. 26, 74-86. doi: 10.1007/BF02111283

O'Connor, D. H., Peron, S. P., Huber, D., and Svoboda, K. (2010). Neural activity in barrel cortex underlying vibrissa-based object localization in mice. Neuron 67, 1048-1061. doi: 10.1016/j.neuron.2010.08.026

Ogawa, S., Lee, T.-M., Nayak, A. S., and Glynn, P. (1990). Oxygenationsensitive contrast in magnetic resonance image of rodent brain at high magnetic fields. Magn. Reson. Med. 14, 68-78. doi: 10.1002/mrm.1910140108

Oh, S., Fang-Yen, C., Choi, W., Yaqoob, Z., Fu, D., Park, Y., et al. (2012). Label-free imaging of membrane potential using membrane electromotility. Biophys. J. 103, 11-18. doi: 10.1016/j.bpj.2012.05.020

Ono, T., and Yano, Y. (1998). Key technologies for terabit/second $\mathrm{wdm}$ systems with high spectral efficiency of over $1 \mathrm{bit} / \mathrm{s} / \mathrm{hz}$. Quantum Electron. IEEE J. 34, 2080-2088. doi: $10.1109 / 3.726596$

Oron, D., Tal, E., and Silberberg, Y. (2005). Scanningless depthresolved microscopy. Opt. Express 13, 1468-1476. doi: 10.1364/OPEX. 13.001468

Ozeri, S., and Shmilovitz, D. (2010). Ultrasonic transcutaneous energy transfer for powering implanted devices. Ultrasonics 50, 556-566. doi: 10.1016/j.ultras.2009.11.004

Packer, A. M., Roska, B., and Häusser, M. (2013). Targeting neurons and photons for optogenetics. Nat. Neurosci. 16, 805-815. doi: 10.1038/nn.3427

Papagiakoumou, E., Anselmi, F., Bègue, A., de Sars, V., Glückstad, J., Isacoff, E. Y., et al. (2010). Scanless two-photon excitation of channelrhodopsin-2. Nat. Methods 7, 848-854. doi: 10.1038/nmeth. 1505

Papagiakoumou, E., Bègue, A., Leshem, B., Schwartz, O., Stell, B. M., Bradley, J., et al. (2013). Functional patterned multiphoton excitation deep inside scattering tissue. Nat. Photon. 7, 274-278. doi: 10.1038/ nphoton.2013.9
Pappu, R., Recht, B., Taylor, J., and Gershenfeld, N. (2002). Physical one-way functions. Science 297, 2026-2030. doi: 10.1126/science. 1074376

Parpaleix, A., Houssen, Y. G., and Charpak, S. (2013). Imaging local neuronal activity by monitoring po2 transients in capillaries. Nat. Med. 19, 241-246. doi: 10.1038/nm.3059

Pedreira, C., Martinez, J., Ison, M. J. and Quian Quiroga, R. (2012). How many neurons can we see with current spike sorting algorithms? J. Neurosci. Methods 211, 58-65. doi: 10.1016/j.jneumeth.2012.07.010

Petridou, N., Plenz, D., Silva, A. C. Loew, M., Bodurka, J., and Bandettini, P. A. (2006). Direct magnetic resonance detection of neuronal electrical activity. Proc. Natl. Acad. Sci. U.S.A. 103, 16015-16020. doi: 10.1073/pnas. 0603219103

Pillow, J. W., Shlens, J., Chichilnisky, E. J., and Simoncelli, E. P. (2013). A model-based spike sorting algorithm for removing correlation artifacts in multi-neuron recordings. PLoS ONE 8:e62123. doi: 10.1371/journal.pone.0062123

Pnevmatikakis, E. A., and Paninski, L. (2013). Sparse nonnegative deconvolution for compressive calcium imaging: algorithms and phase transitions. Adv. Neural Inf. Process. Syst. 1-14.

Polderman, K. H. (2004). Application of therapeutic hypothermia in the ICU: opportunities and pitfalls of a promising treatment modality. Part 1: indications and evidence. Intensive Care Med. 30, 556-575. doi: 10.1007/s00134-0032152-X

Polikov, V. S., Tresco, P. A., and Reichert, W. M. (2005). Response of brain tissue to chronically implanted neural electrodes. J. Neurosci. Methods 148, 1-18. doi: 10.1016/j.jneumeth.2005.08.015

Popoff, S. M., Lerosey, G., Carminati, R., Fink, M., Boccara, A. C., and Gigan, S. (2010). Measuring the transmission matrix in optics. Phys. Rev. Lett. 104:e100601. doi: 10.1103/PhysRevLett.104.100601

Prentice, J. S., Homann, J., Simmons, K. D., Tkacik, G., Balasubramanian, V., and Nelson, P. C. (2011). Fast, scalable, bayesian spike identification for multi-electrode arrays. PLoS ONE 6:e19884. doi: 10.1371/journal.pone.0019884

Priya, S., Ryu, J., Park, C.-S., Oliver, J., Choi, J.-J., and Park, D.-S. (2009). Piezoelectric and magnetoelectric thick films for fabricating power sources in wireless sensor nodes. Sensors 9, 6362-6384. doi: 10.3390/s90806362

Quirin, S., Peterka, D. S., and Yuste, R. (2013). Instantaneous threedimensional sensing using spatial light modulator illumination with extended depth of field imaging. Optics Exp. 21, 16007-16021. doi: 10.1364/OE.21.016007

Rapoport, B. I., Kedzierski, J. T., and Sarpeshkar, R. (2012). A glucose fuel cell for implantable brain-machine interfaces. PLoS ONE 7:e38436. doi: 10.1371/journal.pone.0038436

Rapoport, B. I., Wattanapanitch, W., Penagos Vargas, H. L., Musallam, S., Andersen, R. A., and Sarpeshkar, R. (2009). A biomimetic adaptive algorithm and low-power architecture for implantable neural decoders. Eng. Med. Biol. Soc. 2009, 4214-4217. doi: 10.1109/IEMBS. 2009.5333793

Raskar, R., and Tumblin, J. (2009). Computational Photography: Mastering New Techniques for Lenses, Lighting, and Sensors. Natick, MA: AK Peters, Ltd.

Reichert, W. M. (2010). Indwelling Neural Implants: Strategies for Contending with the in vivo Environment. Boca Raton, FL: CRC Press.

Reichert, W. M., He, W., and Bellamkonda, R. V. (2008). "A molecular perspective on understanding and modulating the performance of chronic central nervous system (CNS) recording electrodes," in Indwelling Neural Implants: Strategies for Contending with the In Vivo Environment, ed W. M. Reichert (Boca Raton, FL: CRC Press).

Reimann, M. W., Anastassiou, C. A., Perin, R., Hill, S. L., Markram, H., and Koch, C. (2013). A biophysically detailed model of neocortical local field potentials predicts the critical role of active membrane currents. Neuron 79, 375-390. doi: 10.1016/j.neuron. 2013.05.023

Reticon Inc. (2013). P Series Linear Photodiode Array Imager Datasheet. Sunnyvale, CA: Reticon, Inc. Available online at: http://www.jai.com/sitecollectionim ages/sensor_datasheet_p-series-021w eb.pdf (Accessed on August 24, 2013).

Ribeiro, S., Gervasoni, D., Soares, E. S., Zhou, Y., Lin, S.-C., Pantoja, J., et al. (2004). Long-lasting noveltyinduced neuronal reverberation during slow-wave sleep in multiple forebrain areas. PLoS Biol. 2:e24. doi: 10.1371/journal.pbio. 0020024 
Rizk, M., Bossetti, C. A., Jochum, T. A., Callender, S. H., Nicolelis, M. A., Turner, D. A., et al. (2009). A fully implantable 96-channel neural data acquisition system. J. Neural Eng. 6:6002. doi: 10.1088/1741-2560/6/2/026002

Rooney, W. D., Johnson, G., Li, X., Cohen, E. R., Kim, S.-G., Ugurbil, K., et al. (2007). Magnetic field and tissue dependencies of human brain longitudinal ${ }^{1} \mathrm{H}_{2} \mathrm{O}$ relaxation in vivo. Magn. Reson. Med. 57, 308-318. doi: 10.1002/ mrm. 21122

Roth, B. J., and Basser, P. J. (2009). Mechanical model of neural tissue displacement during lorentz effect imaging. Mag. Reson. Med. 61, 59-64. doi: $10.1002 / \mathrm{mrm}$. 21772

Roxin, A., Brunel, N., Hansel, D., Mongillo, G., and van Vreeswijk, C. (2011). On the distribution of firing rates in networks of cortical neurons. J. Neurosci. 31, 16217-16226. doi: 10.1523/JNEUROSCI.1677-11.2011

Sadek, A. S., Karabalin, R. B., Du, J., Roukes, M. L., Koch, C., and Masmanidis, S. C. (2010). Wiring nanoscale biosensors with piezoelectric nanomechanical resonators. Nano Lett. 10, 1769-1773. doi: $10.1021 / \mathrm{nl} 100245 \mathrm{z}$

Sahani, M. (1999). Latent Variable Models for Neural Data Analysis, $\mathrm{PhD}$ Dissertation (Pasadena, CA: California Institute of Technology). Available online at: http://www.gatsby.ucl.ac.uk/ manee sh/thesis/thesis.double.pdf

Sanjana, N. E., Levanon, E. Y., Hueske, E. A., Ambrose, J. M., and $\mathrm{Li}$, J. B. (2012). Activitydependent a-to-i rna editing in rat cortical neurons. Genetics 192, 281-287. doi: 10.1534/genetics.112. 141200

Sarpeshkar, R. (1998). Analog versus digital: extrapolating from electronics to neurobiology. Neural Comput. 10, 1601-1638. doi: 10.1162/089976698300017052

Sarpeshkar, R. (2010). Ultra Low Power Bioelectronics. New York, NY: Cambridge University Press. doi: 10.1017/CBO9780511841446

Scanziani, M., and Häusser, M. (2009). Electrophysiology in the age of light. Nature 461, 930-939. doi: 10.1038 /nature 08540

Schmidt, R. F., and Thews, G. (eds.). (1989). Human Physiology, 2nd Edn. New York, NY: Springer-Verlag, 11. Available online at: http://www. amazon.com/dp/0387194320/

Schneidman, E., Berry, M. J., Segev, R., and Bialek, W. (2006). Weak pairwise correlations imply strongly correlated network states in a neural population. Nature 440, 1007-1012. doi: 10.1038/nature04701

Schrödel, T., Prevedel, R., Aumayr, K., Zimmer, M., and Vaziri, A. (2013). Brain-wide 3D imaging of neuronal activity in Caenorhabditis elegans with sculpted light. Nat. Methods 10, 1013-1020. doi: 10.1038/nmeth. 2637

Segev, R., Goodhouse, J., Puchalla, J., and Berry, II, M. J. (2004). Recording spikes from a large fraction of the ganglion cells in a retinal patch. Nat. Neurosci. 7, 1155-1162. doi: 10.1038/nn1323

Sela, G., Dana, H., and Shoham, S. (2013). "Ultra-deep penetration of temporally-focused twophoton excitation," in SPIE BiOS, International Society for Optics and Photonics, eds A. Periasamy, K. König, and P. T. C. So (San Francisco, CA), 858824.

Seliger, H. H., and McElroy, W. D. (1960). Spectral emission and quantum yield of firefly bioluminescence. Arch. Biochem. Biophys. 88, 136-141. doi: 10.1016/0003-9861(60)90208-3

Seo, D., Carmena, J. M., Rabaey, J. M., Alon, E., and Maharbiz, M. M. (2013). Neural dust: an ultrasonic, low power solution for chronic brain-machine interfaces. eprint: arXiv:1307.2196

Shafi, M., Zhou, Y., Quintana, J., Chow, C., Fuster, J., and Bodner, M. (2007). Variability in neuronal activity in primate cortex during working memory tasks. Neuroscience 146, 1082-1108. doi: 10.1016/j.neuroscience.2006.12.072

Shapiro, M. G., Atanasijevic, T., Faas, H., Westmeyer, G. G., and Jasanoff, A. (2006). Dynamic imaging with MRI contrast agents: quantitative considerations. Magn. Res. Imaging 24, 449-462. doi: 10.1016/j.mri.2005.12.033

Shapiro, M. G., Homma, K., Villarreal, S., Richter, C.-P., and Bezanilla, F. (2012). Infrared light excites cells by changing their electrical capacitance. Nat. Commun. 3. doi: 10.1038/ncomms 1742

Shapiro, M. G., Priest, M. F., Siegel, P. H., and Bezanilla, F. (2013). Temperature-mediated effects of millimeter wave stimulation on membrane protein function. Biophys. J. 104:679a. doi: 10.1016/j.bpj.2012.11.3747

Shapiro, M. G., Westmeyer, G. G., Romero, P. A., Szablowski, J. O., Küster, B., Shah, A., (2010). Directed evolution of a magnetic resonance imaging contrast agent for noninvasive imaging of dopamine. Nat. Biotechnol. 28, 264-270. doi: 10.1038/nbt.1609

Shcherbakova, D. M., and Verkhusha, V. V. (2013). Near-infrared fluorescent proteins for multicolor in vivo imaging. Nat Methods. 10, 751-754. doi: 10.1038/nmeth.2521

Shcherbo, D., Murphy, C. S., Ermakova, G. V., Solovieva, E. A., Chepurnykh, T. V., Shcheglov, A. S., et al. (2009). Far-red fluorescent tags for protein imaging in living tissues. Biochem. J. 418, 567-574. doi 10.1042/BJ20081949

Shiu, D.-s., Foschini, G. J., Gans, M. J., and Kahn, J. M. (2000). Fading correlation and its effect on the capacity of multielement antenna systems. IEEE Trans. Commun. 48, 502-513. doi: 10.1109/26.837052

Shoham, S., O'Connor, D. H., and Segev, R. (2006). How silent is the brain: is there a dark matter problem in neuroscience? $J$ Comp. Physiol. A 192, 777-784. doi: 10.1007/s00359-006-0117-6

Silver, I. A., and Erecińska, M. (1994). Extracellular glucose concentration in mammalian brain: continuous monitoring of changes during increased neuronal activity and upon limitation in oxygen supply in normo-, hypo-, and hyperglycemic animals. J. Neurosci. 14, 5068-5076.

Sirotin, Y. B., and Das, A. (2009). Anticipatory haemodynamic signals in sensory cortex not predicted by local neuronal activity. Nature 457, 475-479. doi: 10.1038/nature 07664

Smetters, D., Majewska, A., and Yuste, R. (1999). Detecting action potentials in neuronal populations with calcium imaging. Methods 18, 215-221. doi: 10.1006/meth.1999.0774

Smith, K. D., and Zhu, L. (2010). Brain hypothermia induced by cold spinal fluid using a torso cooling pad: theoretical analyses. Med. Biol. Eng. comput. 48, 783-791. doi 10.1007/s11517-010-0635-9

Sotero, R. C., and Iturria-Medina, Y. (2011). From blood oxygenation level dependent (BOLD) signals to brain temperature maps. Bull. Math. Biol. 73, 2731-2747. doi: 10.1007/s11538-011-9645-5

Speakman, J. R. (2013). Measuring energy metabolism in the mouse-theoretical, practical, and analytical considerations. Front. Integr. Physiol. 4:34. doi: 10.3389/fphys.2013.00034

Spencer, Q. H., Peel, C. B., Swindlehurst, A. L., and Haardt, M. (2004). An introduction to the multi-user MIMO downlink. IEEE
Commun. Mag. 42, 60-67. doi: 10.1109/MCOM.2004.1341262

Spruston, N. (2001). Axonal gap junctions send ripples through the hippocampus. Neuron 31, 669-671. doi: 10.1016/ S0896-6273(01)00426-3

Stehling, M. K., Turner, R., and Mansfield, P. (1991). Science 254, 43-50. doi: 10.1126/science.192 5560

Stepnoski, R., LaPorta, A., RaccuiaBehling, F., Blonder, G., Slusher, R., and Kleinfeld, D. (1991) Noninvasive detection of changes in membrane potential in cultured neurons by light scattering. Proc. Natl. Acad. Sci. U.S.A. 88, 9382-9386. doi: 10.1073/pnas.88. 21.9382

Stevenson, I. H., and Kording, K. P. (2011). How advances in neural recording affect data analysis. Nat. Neurosci. 14, 139-142. doi: $10.1038 / \mathrm{nn} .2731$

Steyaert, M. S. J., Sansen, W. M. C., and Chang, Z.-Y. (1987). A micropower low-noise monolithic instrumentation amplifier for medical purposes. IEEE J. SolidState Circ. 22, 1163-1168. doi: 10.1109/JSSC.1987.1052869

Storace, D. A., Sung, U., Platisa, J., Cohen, L. B., and Pieribone, V. A. (2013). In vivo imaging of odorevoked responses in the olfactory bulb using arclight, a novel Fp voltage probe. Biophys. J. 104:679. doi: 10.1016/j.bpj.2012.11.3751

Stratton, P., Cheung, A., Wiles, J., Kiyatkin, E., Sah, P., and Windels, F. (2012). Action potential waveform variability limits multi-unit separation in freely behaving rats. PLoS ONE 7:e38482. doi: 10.1371/journal.pone.0038482

Striker, G., Subramaniam, V., Seidel, C. A. M., and Volkmer, A. (1999). Photochromicity and fluorescence lifetimes of green fluorescent protein. J. Phys. Chem. B 103, 8612-8617. doi: $10.1021 /$ jp991425e

Strong, S. P., Koberle, R., de Ruyter van Steveninck, R. R., and Bialek, W. (1998). Entropy and information in neural spike trains. Phys. Rev. Lett. 80, 197-200. doi: 10.1103/PhysRevLett.80.197

Studer, V., Bobin, J., Chahid, M., Mousavi, H. S., Candes, E., and Dahan, M. (2012). Compressive fluorescence microscopy for biological and hyperspectral imaging. Proc. Natl. Acad. Sci. U.S.A. 109, E1679-E1687. doi: 10.1073/pnas.1119511109

Sukstanskii, A., and Yablonskiy, D. (2004). An analytical model 
of temperature regulation in human head. J. Thermal Biol. 29, 583-587. doi: 10.1016/j.jtherbio. 2004.08.028

Sukstanskii, A., and Yablonskiy, D. (2007). Theoretical limits on brain cooling by external head cooling devices. Eur. J. Appl. Physiol. 101, 41-49. doi: 10.1007/s00421007-0452-5

Sukstanskii, A. L., and Yablonskiy, D. A. (2006). Theoretical model of temperature regulation in the brain during changes in functional activity. Proc. Natl. Acad. Sci. U.S.A. 103, 12144-12149. doi: 10.1073/pnas.0604376103

Sun, B., Edgar, M. P., Bowman, R., Vittert, L. E., Welsh, S., Bowman, A., et al. (2013a). 3D computational imaging with single-pixel detectors. Science 340, 844-847. doi: 10.1126/science. 1234454

Sun, X. R., Badura, A., Pacheco, D. A., Lynch, L. A., Schneider, E. R., Taylor, M. P., et al. (2013b). Fast gcamps for improved tracking of neuronal activity. Nat. Commun. 4. doi: 10.1038/ncomms3170

Sun, Y.-H., and Chang, K. (2002). A high-efficiency dual-frequency rectenna for $2.45-$ and $5.8-\mathrm{GHz}$ wireless power transmission. IEEE Trans. Microw. Theory Tech. 50, 1784-1789. doi: 10.1109/TMTT.2002.800430

Suner, S., Fellows, M. R., Vargas-Irwin, C., Nakata, G. K., and Donoghue, J. P. (2005). Reliability of signals from a chronically implanted, silicon-based electrode array in non-human primate primary motor cortex. Neural Syst. Rehabil. Eng. IEEE Trans. 13, 524-541. doi: 10.1109/TNSRE.2005.857687

Taillefumier, T., and Magnasco, M. O. (2013). A phase transition in the first passage of a brownian process through a fluctuating boundary with implications for neural coding. Proc. Natl. Acad. Sci. U.S.A. 110, E1438-E1443. doi: 10.1073/ pnas. 1212479110

Tal, E., Oron, D., and Silberberg, Y. (2005). Improved depth resolution in video-rate line-scanning multiphoton microscopy using temporal focusing. Opt. Lett. 30, 1686-1688. doi: 10.1364/OL.30.001686

Taub, A. H., Hogri, R., Magal, A., Mintz, M., and ShachamDiamand, Y. (2012). Bioactive anti-inflammatory coating for chronic neural electrodes. J. Biomed. Mater. Res. A 100, 1854-1858. doi: 10.1002/jbm.a.34152

Tian, N., Guo, Q., Wang, A., Xu, D., and $\mathrm{Fu}$, L. (2011). Fluorescence ghost imaging with pseudothermal light. Opt. Lett. 36, 3302-3304. doi: 10.1364/OL.36. 003302

Tonouchi, M. (2007). Cutting-edge terahertz technology. Nat. Photon. 1, 97-105. doi: 10.1038/nphoton. 2007.3

Trübel, H. K., Sacolick, L. I., and Hyder, F. (2005). Regional temperature changes in the brain during somatosensory stimulation. J. Cereb. Blood Flow Metab. 26, 68-78. doi: 10.1038/sj.jcbfm. 9600164

Tsia, K. K., Goda, K., Capewell, D., and Jalali, B. (2010). "Performance of serial time-encoded amplified microscopy," in Lasers and ElectroOptics (CLEO) and Quantum Electronics and Laser Science Conference (QELS), 2010 Conference on (San Jose, CA: IEEE), 1-2.

Tsurugizawa, T., Ciobanu, L., and Le Bihan, D. (2013). Water diffusion in brain cortex closely tracks underlying neuronal activity. Proc. Natl. Acad. Sci. U.S.A. 110, 11636-11641. doi: 10.1073/pnas. 1303178110

Tucker, R. S. (2011). Green optical communications-Part II: energy limitations in networks. IEEE J. Select. Topics Quant. Electron. 17, 261-274. doi: 10.1109/JSTQE.2010.2051217

Tucker, R. S., and Hinton, K. (2011). Energy consumption and energy density in optical and electronic signal processing. IEEE Photon. J. 3, 821-833. doi: 10.1109/JPHOT.2011.2166254

Tulino, A. M., and Verdú, S. (2004) Random matrix theory and wireless communications. Found. Trends Commun. Inf. Theory 1, 1-182. doi: 10.1561/0100000001

Usami, M., Tanabe, H., Sato, A. Sakama, I., Maki, Y., Iwamatsu, T., et al. (2007). "A $0.05 \times 0.05 \mathrm{~mm}^{2}$ RFID chip with easily scaled-down ID-memory," in International Solid-State Circuits Conference, 2007 IEEE, (San Francisco, CA), 482-483.

Vacher, H., Mohapatra, D. P., and Trimmer, J. S. (2008). Localization and targeting of voltage-dependent ion channels in mammalian central neurons. Physiol. Rev. 88, 1407-1447. doi: 10.1152/physrev.00002.2008

Van der Linden, A., Verhoye, $M$. Van Meir, V., Tindemans, I., Eens, M., Absil, P., et al. (2002). In vivo manganese-enhanced magnetic resonance imaging reveals connections and functional properties of the songbird vocal control system.
Neuroscience 112, 467-474. doi 10.1016/S0306-4522(02)00070-2

Vaziri, A., and Emiliani, V. (2012) Reshaping the optical dimension in optogenetics. Curr. Opin. Neurobiol. 22, 128-137. doi: 10.1016/j.conb.2011.11.011

Velten, A., Willwacher, T., Gupta, O., Veeraraghavan, A., Bawendi, M. G. and Raskar, R. (2012). Recovering three-dimensional shape around a corner using ultrafast time-of-flight imaging. Nat. Commun. 3:745. doi: 10.1038/ncomms 1747

Vetter, R. J., Williams, J. C., Hetke, J. F., Nunamaker, E. A., and Kipke, D. R. (2004). Chronic neura recording using silicon-substrate microelectrode arrays implanted in cerebral cortex. Biomed. Eng. IEEE Trans. 51, 896-904. doi: 10.1109/TBME. 2004.826680

Vincent, T. J., Thiessen, J. D., Kurjewicz L. M., Germscheid, S. L., Turner, A. J., Zhilkin, P., et al. (2010). Longitudinal brain size measurements in APP/PS1 transgenic mice. Magn. Reson. Insights 4, 19-26. doi: 10.4137/MRI.S5885

Vučinić, D., and Sejnowski, T. J. (2007). A compact multiphoton $3 \mathrm{~d}$ imaging system for recording fast neuronal activity. PLOS ONE 2:e699. doi: 10.1371/journal.pone. 0000699

Wakin, M. B., Laska, J. N., Duarte, M. F., Baron, D., Sarvotham, S., Takhar, D., et al. (2006). "Compressive imaging for video representation and coding," in Picture Coding Symposium. (Beijing).

Wang, Y. M., Judkewitz, B., DiMarzio C. A., and Yang, C. (2012). Deeptissue focal fluorescence imaging with digitally time-reversed ultrasound-encoded light. Nat. Commun. 3. doi: 10.1038/ncomms 1925

Ward, M. P., Rajdev, P., Ellison, C. and Irazoqui, P. P. (2009). Toward a comparison of microelectrodes for acute and chronic recordings. Brain Res. 1282, 183-200. doi: 10.1016/j.brainres.2009.05.052

Wee, T.-L., Tzeng, Y.-K., Han, C.-C., Chang, H.-C., Fann, W., Hsu, J.-H., et al. (2007). Two-photon excited fluorescence of nitrogen-vacancy centers in proton-irradiated type ib diamond. J. Phys. Chem. A 111, 9379-9386. doi: 10.1021/ jp073938o

Weibel, D. B., Garstecki, P., Ryan, D., DiLuzio, W. R., Mayer, M., Seto, J. E., et al. (2005). Microoxen. Proc. Natl. Acad. Sci. U.S.A. 102 11963-11967. doi: 10.1073/pnas. 0505481102
Wessberg, J., Stambaugh, C. R., Kralik, J. D., Beck, P. D., Laubach, M., Chapin, J. K., et al. (2000). Realtime prediction of hand trajectory by ensembles of cortical neurons in primates. Nature 408. doi: $10.1038 / 35042582$

Widder, E. A., Latz, M. I., Herring, P. J., and Case, J. F. (1984). Far red bioluminescence from two deep-sea fishes. Science 225, 512-514. doi: 10.1126/science.225.4661.512

Wiesinger, F., Van de Moortele, P.F., Adriany, G., De Zanche, N., Ugurbil, K., and Prüssman, K. P. (2006). Potential and feasibility of parallel MRI at high field. NMR Biomed. 19, 368-378. doi: 10.1002/nbm. 1050

Wilson, J. M., Dombeck, D. A. Díaz-Ríos, M., Harris-Warrick, R. M., and Brownstone, R. M. (2007). Two-photon calcium imaging of network activity in XFPexpressing neurons in the mouse. J. Neurophysiol. 97, 3118-3125. doi: 10.1152/jn.01207.2006

Wilt, B. A., Burns, L. D., Wei Ho, E. T., Ghosh, K. K., Mukamel, E. A., and Schnitzer, M. J. (2009). Advances in light microscopy for neuroscience. Annu. Rev. Neurosci. 32, 435-506. doi: 10.1146/annurev.neuro.051508. 135540

Wilt, B. A., Fitzgerald, J. E., and Schnitzer, M. J. (2013). Photon shot noise limits on optical detection of neuronal spikes and estimation of spike timing. Biophys. J. 104, 51-62. doi: 10.1016/j.bpj. 2012.07.058

Witzel, T., Lin, F.-H., Rosen, B. R. and Wald, L. L. (2008). Stimulusinduced rotary saturation (sirs): a potential method for the detection of neuronal currents with mri. Neuroimage 42, 1357-1365. doi: 10.1016/j.neuroimage.2008.05.010

Wolf, P. D. (2008). "Thermal considerations for the design of an implanted cortical brain-machine interface (BMI)," in Indwelling Neural Implants: Strategies for Contending with the In Vivo Environment, ed W. M. Reichert (Boca Raton, FL: CRC Press).

Wood, L. (2013). Personal Communication.

Wu, F., Zhang, X., Cheung, J. Y., Shi, K., Liu, Z., Luo, C., et al. (2006). Frequency division multiplexed multichannel high-speed fluorescence confocal microscope. Biophys. J. 91, 2290-2296. doi: 10.1529/biophysj.106.083337

Xu, T.-B., Siochi, E. J., Kang, J. H., Zuo, L., Zhou, W., Tang, X., et al. (2012). Energy harvesting using a PZT ceramic multilayer stack. 
Smart Mater. Struct. 22:065015. doi: 10.1088/0964-1726/22/6/065015

Yablonovitch, E. (2008). Replacing the transistor: searching for the milli-volt switch. Available online at: http://ustlib.ust.hk/ record=b1161099

Yamagata, M., and Sanes, J. R. (2012). Transgenic strategy for identifying synaptic connections in mice by fluorescence complementation (grasp). Front. Mol. Neurosci. 5:18. doi: 10.3389/fnmol.2012.00018

Yin, S. (2006). Frequency division multiplexed fluorescence confocal microscopy. Biophoton. Int. 13:38.

Yu, X., Glen, D., Wang, S., Dodd, S., Hirano, Y., Saad, Z., et al. (2012). Direct imaging of macrovascular and microvascular contributions to BOLD fMRI in layers IV-V of the rat whisker-barrel cortex. Neuroimage 59, 1451-1460. doi: 10.1016/j.neuroimage.2011.08.001

Yue, K., Guduru, R., HongJeongmin, Liang, P., Nair, M., and Khizroev, S. (2012). Magneto-electric nano-particles for non-invasive brain stimulation. PLoS ONE 7:e44040. doi: 10.1371/journal. pone. 0044040

Zador, A. M., Dubnau, J., Oyibo, H. K., Zhan, H., Cao, G., and Peikon, I. D. (2012). Sequencing the connectome. PLoS Biol. 10:e1001411. doi: 10.1371/ journal.pbio.1001411

Zamft, B. M., Marblestone, A. H., Kording, K. P., Schmidt, D., Martin-Alarcon, D., Tyo, K., et al. (2012). Measuring cation dependent DNA polymerase fidelity landscapes by deep sequencing. PLoS ONE 7:e43876. doi: 10.1371/journal.pone.0043876

Zhang, S., Block, K. T., and Frahm, J. (2010). Magnetic resonance imaging in real time. J. Magn. Reson. Imaging 31, 101-109. doi: 10.1002/jmri.21987

Ziv, Y., Burns, L. D., Cocker, E. D., Hamel, E. O., Ghosh, K. K., Kitch, L. J., et al. (2013). Long-term dynamics of CAl hippocampal place codes. Nat. Neurosci. 16, 264-266. doi: 10.1038/nn.3329
Zorzos, A. N., Boyden, E. S., and Fonstad, C. G. (2010). Multiwaveguide implantable probe for light delivery to sets of distributed brain targets. Opt. Lett. 35, 4133-4135. doi: 10.1364/OL.35.004133

Zorzos, A. N., Scholvin, J., Boyden, E. S., and Fonstad, C. G. (2012). Three-dimensional multiwaveguide probe array for light delivery to distributed brain circuits. Opt. Lett. 37, 4841-4843. doi: 10.1364/OL.37.004841

Conflict of Interest Statement: The authors declare that the research was conducted in the absence of any commercial or financial relationships that could be construed as a potential conflict of interest.

Received: 07 July 2013; accepted: 23 September 2013; published online: 21 October 2013

Citation: Marblestone AH, Zamft BM, Maguire YG, Shapiro MG, Cybulski
TR, Glaser JI, Amodei D, Stranges $P$, Kalhor R, Dalrymple DA, Seo D, Alon E, Maharbiz MM, Carmena JM, Rabaey JM, Boyden ES, Church GM and Kording KP (2013) Physical principles for scalable neural recording. Front. Comput. Neurosci. 7:137. doi: 10.3389/ fncom.2013.00137

This article was submitted to the journal Frontiers in Computational Neuroscience.

Copyright () 2013 Marblestone, Zamft, Maguire, Shapiro, Cybulski, Glaser, Amodei, Stranges, Kalhor, Dalrymple, Seo, Alon, Maharbiz, Carmena, Rabaey Boyden, Church and Kording. This is an open-access article distributed under the terms of the Creative Commons Attribution License (CC BY). The use, distribution or reproduction in other forums is permitted, provided the original author(s) or licensor are credited and that the original publication in this journal is cited, in accordance with accepted academic practice. No use, distribution or reproduction is permitted which does not comply with these terms. 